\title{
Novel Azalides Derived from Sixteen-Membered Macrolides
}

\author{
I. Isolation of the Mobile Dialdehyde and Its One-Pot Macrocyclization \\ with an Amine
}

\author{
Tomoaki Miura, Satomi Natsume, Kenichi Kanemoto, Kunio Atsumi, Hideki Fushimi, \\ Hiroaki Sasai, Takayoshi Arai, Takuji Yoshida, Keiichi Ajito
}

Received: March 28, 2007 / Accepted: June 8, 2007

(C) Japan Antibiotics Research Association

\begin{abstract}
The design and synthesis of novel 15membered 11-azalides and 16-membered 11,12-diazalide starting from 16-membered macrolides are reported. A mobile linear dialdehyde was isolated via a cyclic tetraol which was prepared by osmium oxidation of a conjugated diene. One-pot macrocyclization of this dialdehyde with an amine or a diamine afforded corresponding 15-membered azalides or 11,12-diazalide. Fundamental SAR studies of 15-membered 11-azalides disclosed their potentiality as a lead molecule for further chemical modifications. For environmental preservation, sustainable chemistry for synthesis of these azalides is also discussed.
\end{abstract}

Keywords azalide, 16-membered macrolide, one-pot macrocyclization, dialdehyde, leucomycin

\section{Introduction}

Macrolide antibiotics [1] are active against Gram-positive bacteria (especially Streptococcus pneumoniae), Moraxella catarrhalis, Haemophillus influenzae, and Mycoplasma pneumoniae, and regarded as very important chemotherapeutics from a clinical viewpoint. Clarithromycin [2] (CAM) and azithromycin [3] (AZM) (Fig. 1), which are representatives of widely used macrolides and are derived

K. Ajito (Corresponding author), T. Miura, S. Natsume, K. Kanemoto, K. Atsumi, H. Fushimi, T. Yoshida: Pharmaceutical Research Center, Meiji Seika Kaisha, Ltd., 760 Morooka-cho, Kohoku-ku, Yokohama, 222-8567 Japan,

E-mail: keiichi_ajito@meiji.co.jp from 14-membered erythromycin, exhibited enhanced antibacterial activities and characteristic pharmacokinetics, respectively. As one of the next generation macrolides, i.e. ketolides, telithromycin [4] (TEL) has recently been launched as an efficient antibiotic which is effective against clinically important pathogens including erythromycinresistant Streptococcus pneumoniae (ERSP). Moreover, the novel ketolide, S-013420 [5] is under the clinical trials in 2006.

Although telithromycin exhibits improved antibacterial activities against resistant bacteria of $S$. pneumoniae with an erm gene, it is not always sufficient. Specifically, it is affected by efflux pump function of resistant bacteria in $S$. pneumoniae, and its safety [6] seriously concerns clinical site especially in US. In 2001, we started a novel drug discovery program applying 16-membered macrolide antibiotics which have been proved to be safe and effective against resistant bacteria of $S$. pneumoniae with efflux pump.

Before we started this research program, we had already established two major pharmacological approaches by medicinal chemistry using 16-membered macrolides. Owing to these approaches, (i) biological stability in 16-membered macrolides was dramatically improved [7] by chemical modification of a neutral sugar moiety, and (ii) antibacterial activities against resistant pathogens

H. Sasai, T. Arai: The Institute of Scientific and Industrial Research (ISIR), Osaka University, 8-1 Mihogaoka, Ibaraki, Osaka, 567-0047 Japan 


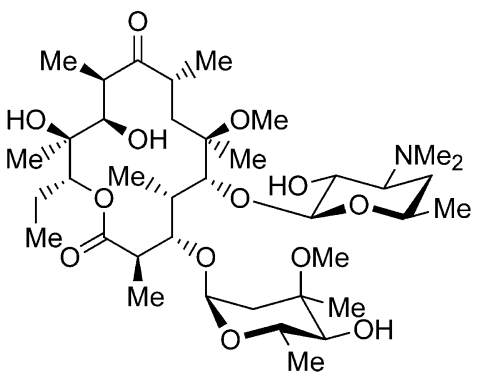

Clarithromycin(CAM)

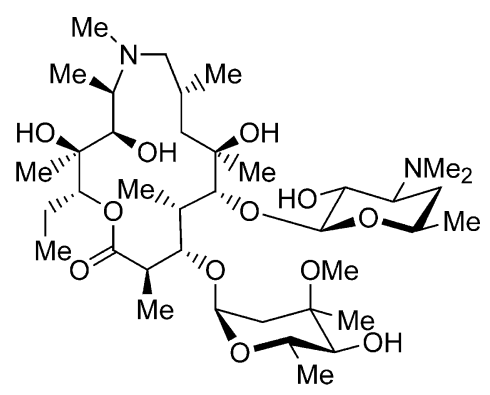

Azithromycin(AZM)

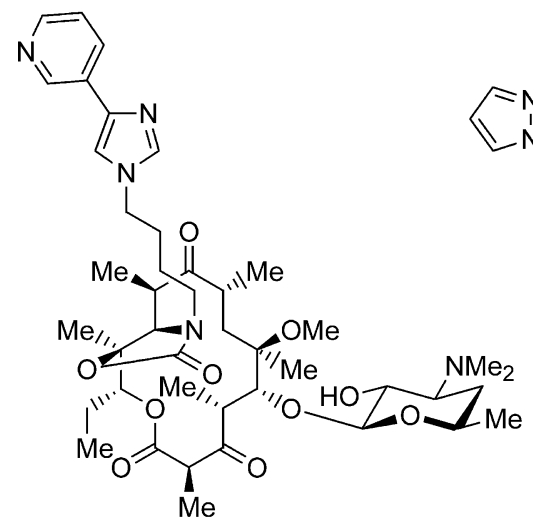

Telithromycin(TEL)

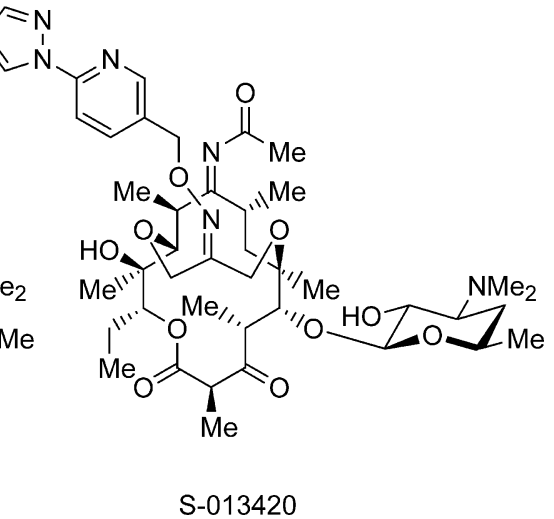

Fig. 1 Structures of representative macrolides.

in 16-membered macrolides was clearly enhanced [8] by introducing an appropriate aromatic ring with an adjusted length of an alkyl spacer into the lactone ring. There is, however, limitation in molecular design and synthesis of novel compounds, as long as we utilize known pharmacophores. We had to explore a novel pharmacophore.

Since the synthesis of azithromycin [9] was reported by Pliva in 1981, many novel azalides were reported in 1990's as shown in Fig. 2 [10 15] and so on [16]. These examples include 13-membered to 17 -membered azalides, but the position of the nitrogen atom in all derivatives is C- 9 or C10 (lactone carbonyl: designated to be C-1). On the other hand, several azalide-based new pharmacophores have been reported in the 21 st century. Pliva disclosed an azalideketolide hybrid molecule [17] with an arylalkyl side chain at the C-6 position. Enanta reported a bicycle azalide [18] which had a bridge between the C-3 and C- 6 positions. Pliva introduced $\mathrm{N}, \mathrm{O}$-carbonate [19] using azithromycin framework. But all of them had the nitrogen atom at the same position as in the derivatives reported in 1990's.

In this paper, we report the design and synthesis of novel 15-membered 11-azalides (9) and 16-membered 11,12-diazalide starting from 16-membered macrolides.
Meanwhile, 12-azalide can be introduced as one of attractive novel azalides disclosed by Taisho in the $21 \mathrm{st}$ century [20].

\section{Results and Discussion}

\section{Isolation of the Mobile Dialdehyde and Its One-Pot Macrocyclization}

Before discussion of synthetic route for 15-membered azalides, an attractive example was reported by Ōmura in 1982 that two large molecules possessing an aldehyde group could reductively react with an amine one by one to afford a dialkylamine, 18,18'-dideoxo-18,18' iminoleucomycin $\mathrm{A}_{3}$ (10) [21] as shown in Fig. 3. Retrosynthetic analysis of 15-membered 11-azalides easily presented a linear dialdehyde (12) as shown in Fig. 4 or its structurally related molecule as a key intermediate. Then, we decided to isolate $\mathbf{1 2}$ and perform its macrocyclization with an amine.

We decided to use an aldehyde as a key intermediate for macrocyclization, and chose dimethoxy acetal protection for the original aldehyde at the $\mathrm{C}-18$ position. Because we had to use difluoroacetic acid [22] to remove this protecting 


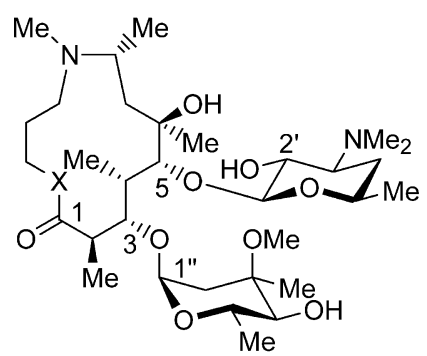

$1^{[10]: X}=\mathrm{O}$ or $\mathrm{NH}$

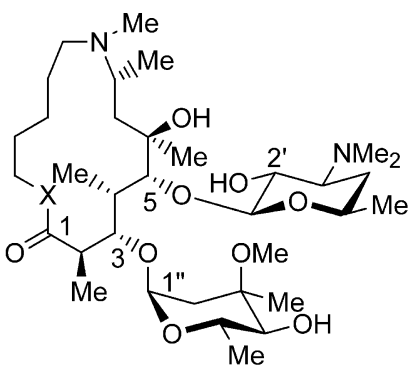

$4^{[10]: X}=\mathrm{O}$ or $\mathrm{NH}$

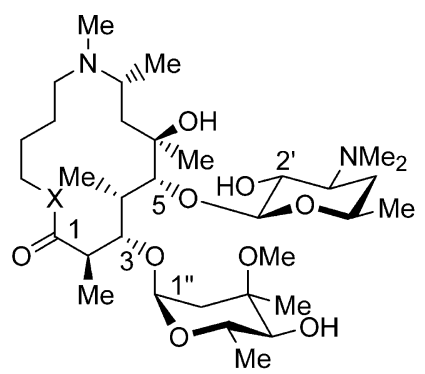

$2^{[10]}: \mathrm{X}=\mathrm{O}$ or $\mathrm{NH}$

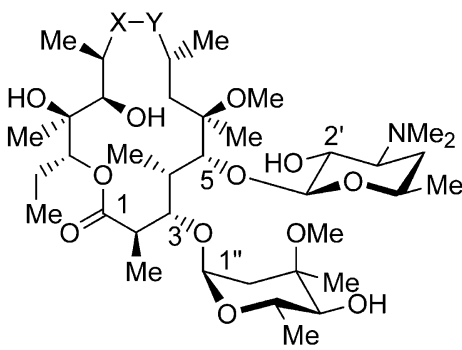

$5^{[12]}: \mathrm{X}=\mathrm{CH}_{2} ; \mathrm{Y}=\mathrm{NH}$

$\mathbf{6 a}^{[13]}: \mathrm{X}=\mathrm{CO} ; \mathrm{Y}=\mathrm{NH}$

$\mathbf{6 b}^{[13]}: \mathrm{X}=\mathrm{NH} ; \mathrm{Y}=\mathrm{CO}$

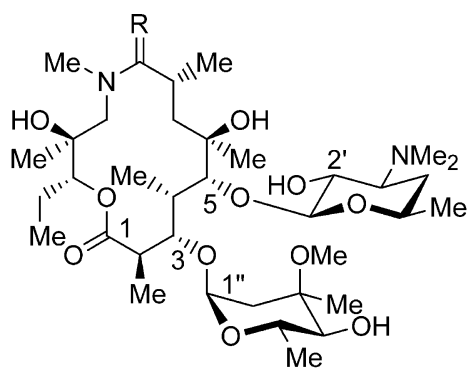

$3^{[11]}: \mathrm{R}=\mathrm{H}, \mathrm{H}$ or O

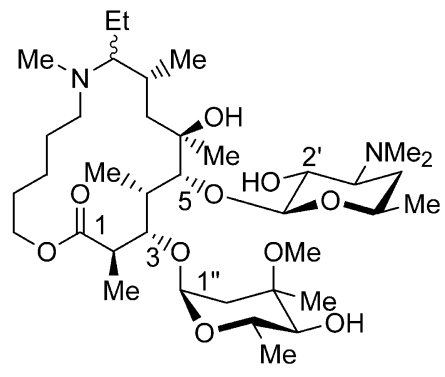

$7^{[14]}$

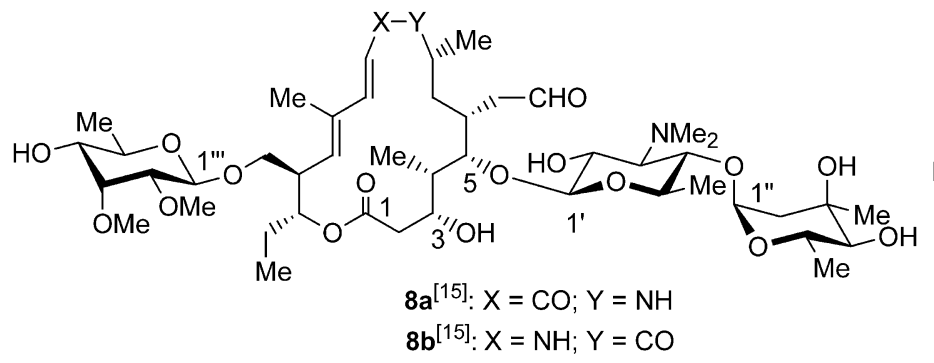

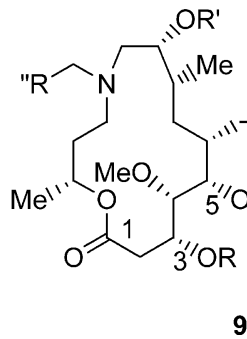

9: Target molecules;

15-membered-11-azalide

Fig. 2 Structures of azalides reported in the 20th century as a novel macrolide framework and target molecules.

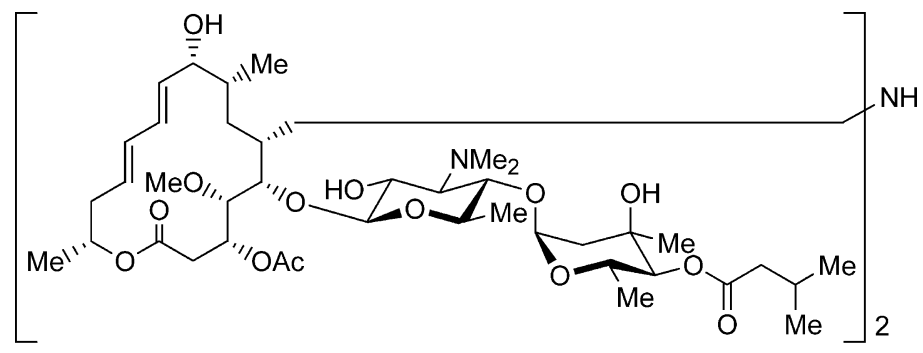

10

Fig. 3 Dialkylamine (10) derived from leucomycin $A_{3}$.

group, we chose miokamycin (MOM [23]) as a starting material (not midecamycin (MDM [24])) (Scheme 1). A neutral sugar moiety in MOM with the 3 "-acetoxy group is more stable against cleavage of a glycoside bond under acidic conditions because of its 1,3-diaxial steric hindrance than that in MDM with the 3"-hydroxyl group. On the other hand, an acetyl group at the C-9 position in MOM is necessary when we utilize a tetraol under oxidation conditions.

Sequential protections of MOM gave $\mathbf{1 5}$, which was converted into the tetraol (13) in a moderate yield by osmium tetroxide with $\mathrm{N}$-methylmorpholine $\mathrm{N}$-oxide in 


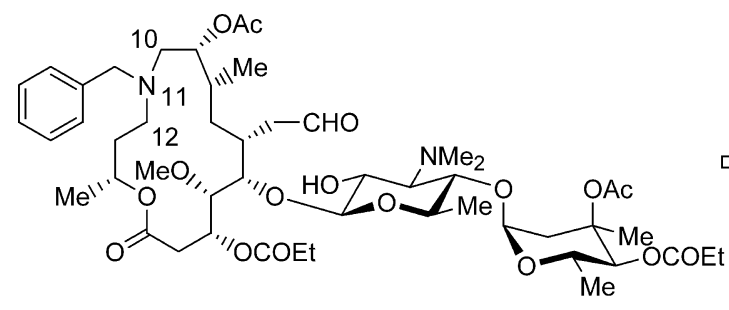

11

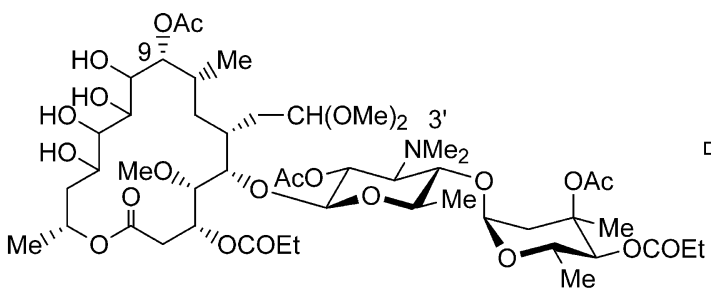

13

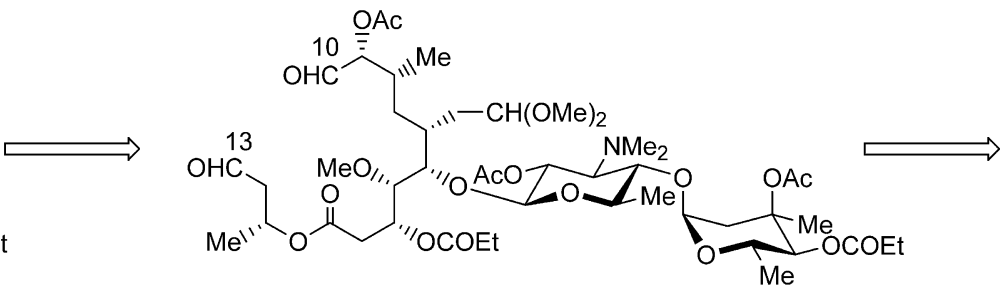

12

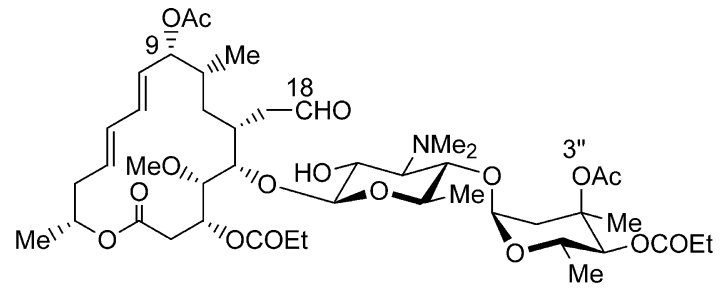

MOM

Fig. 4 Retrosynthetic analysis of compound (11), a prototype of 15-membered azalides.

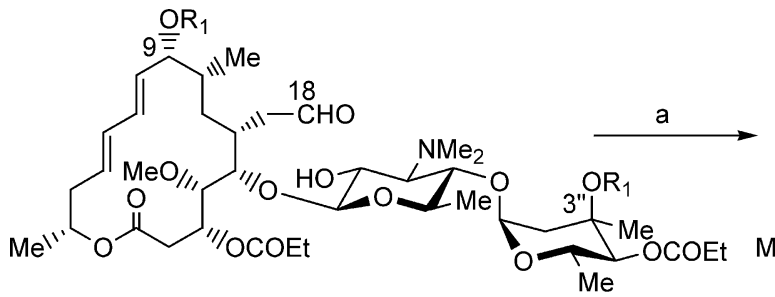
MOM: $R_{1}=A C$ (MDM: $\mathrm{R}_{1}=\mathrm{H}$ )

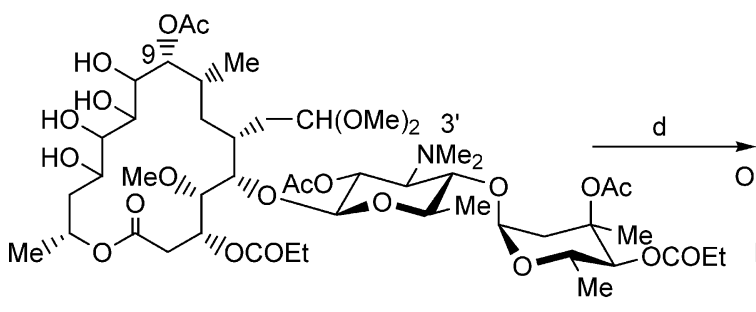

13

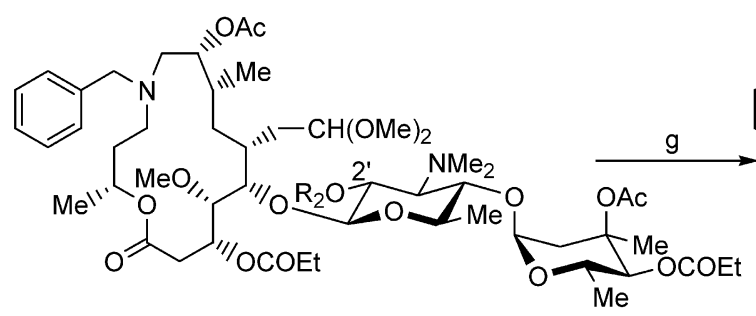

$\begin{aligned} f \longrightarrow \text { 16: } R_{2} & =A C \\ \text { 17: } R_{2} & =H\end{aligned}$

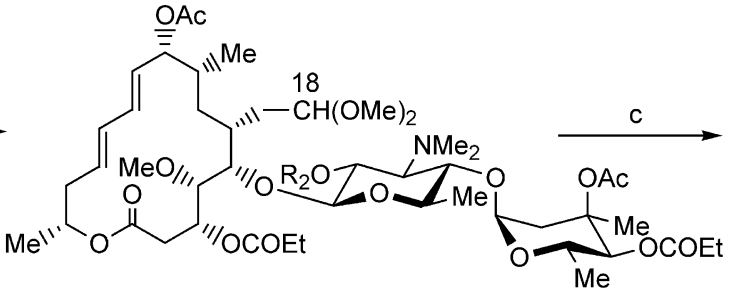

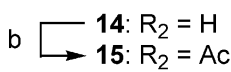

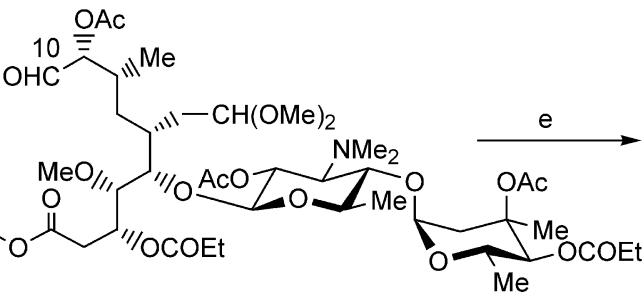

12

11

Reagents and conditions: (a): ( $\mathrm{MeO})_{3} \mathrm{CH}(80 \mathrm{eq}), \mathrm{PPTS}(1.2 \mathrm{eq}), \mathrm{MeOH}, 40 \sim 50^{\circ} \mathrm{C}, 4$ days; (b): $\mathrm{Ac}_{2} \mathrm{O}\left(5\right.$ eq), $\mathrm{MeCN}, 40^{\circ} \mathrm{C}, 16 \mathrm{hours}$; (c): $\mathrm{OsO}_{4}\left(0.15\right.$ eq), $\mathrm{NMO}(2 \mathrm{eq})$, aq. acetone, r.t., 24 hours, $30 \%$ in 3 steps; (d): $\mathrm{Pb}(\mathrm{OAc})_{4}(2.1 \mathrm{eq}), \mathrm{PhH}_{1} \mathrm{Na}_{2} \mathrm{CO}_{3}\left(8\right.$ eq), r.t., 1 hour; $(\mathrm{e}): \mathrm{BnNH}_{2}$ $(1.1 \mathrm{eq}), \mathrm{NaB}(\mathrm{CN}) \mathrm{H}_{3}(3.9 \mathrm{eq}), \mathrm{AcOH}(15 \mathrm{eq}), \mathrm{EtOH}, 0^{\circ} \mathrm{C}$ to r.t., $15 \sim 20$ hours, $10 \%$ in 2 steps; (f): aq $\mathrm{MeOH}, 55^{\circ} \mathrm{C}, 24 \mathrm{hours}, 79 \sim 90 \%,(\mathrm{~g})$ : difluoroacetic acid (20 eq), $\mathrm{MeCN}-\mathrm{H}_{2} \mathrm{O}$, r.t., 25 hours, $84 \sim 94 \%$.

Scheme 1 Isolation of mobile dialdehyde (12) and its macrocyclization with amine or diamine. 


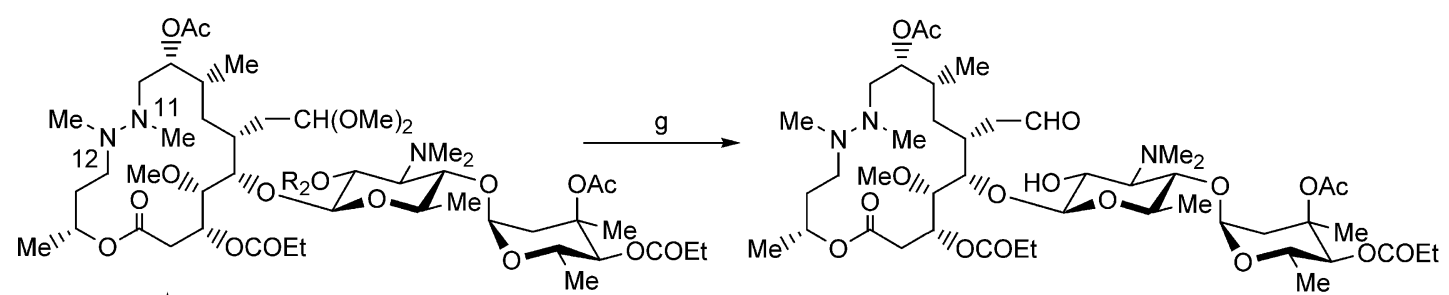

20

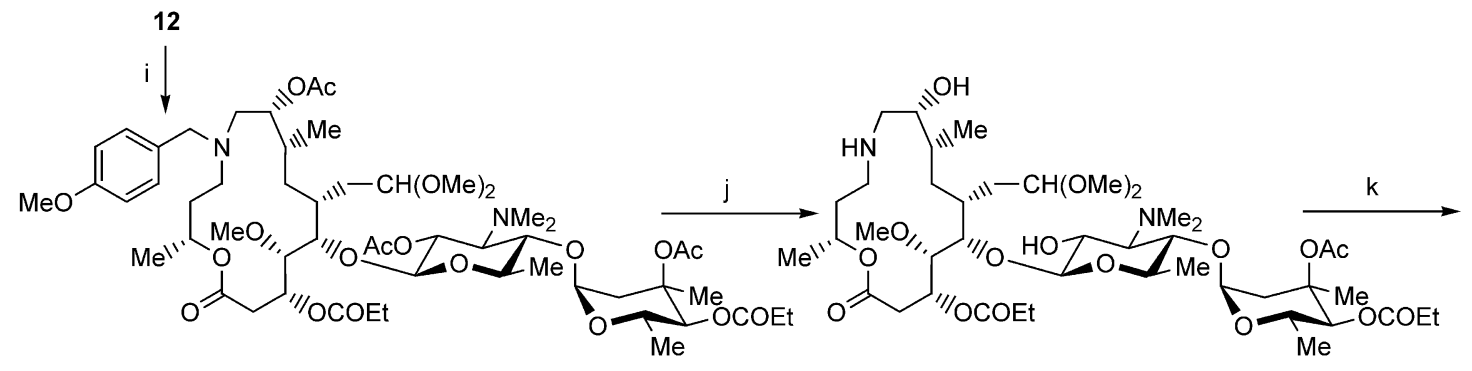

21

22

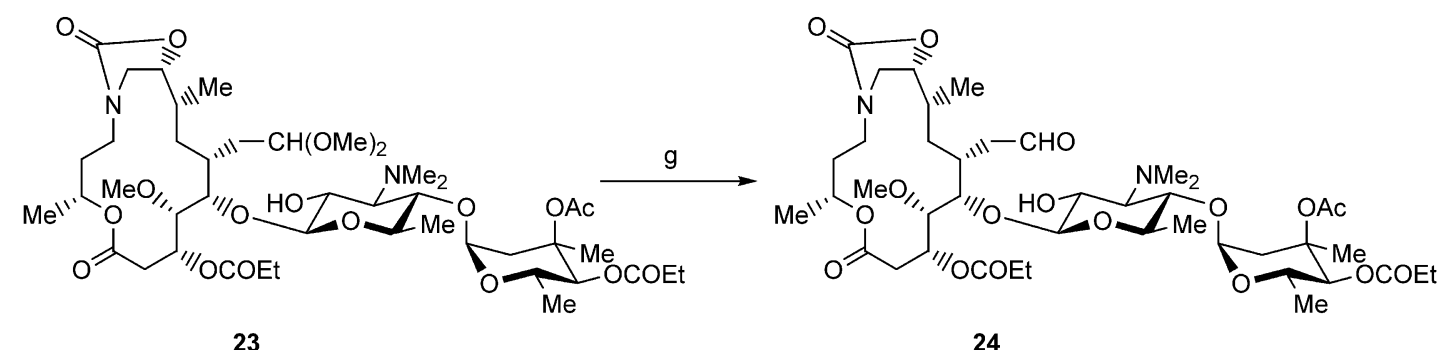

Reagents and conditions: (h): 1,2-dimethylhydrazine hydrochloride (1.1 eq), others are same as (e), 13\%; (i): p-methoxybenzylamine (1.1 eq), others are same as (e), 8.8\%; (j): aq $\mathrm{MeOH}, 55^{\circ} \mathrm{C}, 96$ hours, then, $\mathrm{H}_{2}, \mathrm{Pd} / \mathrm{C}$, dioxane-EtOH, $30 \%$ in 2 steps; (k): triphosgene (1.2 eq), $\mathrm{Et}_{3} \mathrm{~N}(10 \mathrm{eq}), \mathrm{CH}_{2} \mathrm{Cl}_{2}, 0^{\circ} \mathrm{C}, 90$ minutes, $74 \%$.

Scheme 1 Isolation of mobile dialdehyde (12) and its macrocyclization with amine or diamine. (continued)

aqueous acetone. In the osmium oxidation step, $3^{\prime}$-de- $N$ methyl derivative can be detected as a minor byproduct, but this can be converted to $\mathbf{1 3}$ by reductive methylation using aqueous formaldehyde and sodium cyanoborohydride in ethanol in the presence of acetic acid. Then, the oxidation of the tetraol using lead (IV) acetate with sodium carbonate in benzene afforded the mobile dialdehyde (12) in a low yield. This aldehyde can be purified with silica gel column chromatography to prepare desired pure product which shows one doublet $(\delta 9.59, \mathrm{H}-10)$ and one triplet $(\delta 9.75$, so called H-13) in ${ }^{1} \mathrm{H}-\mathrm{NMR}$ spectroscopy. We did not, however, always purify this dialdehyde for further analogue synthesis. Macrocyclization between $\mathbf{1 2}$ and benzylamine with sodium cyanoborohydride in ethanol in the presence of acetic acid gave 15-membered 11-azalide (16) as the first example [25]. The two-step yield was around 10\% from 13 . Deprotection of an acetyl group and dimethylacetal gave a novel macrolide, i.e. 15-membered azalide (11) as a prototype. The acetyl group at the $\mathrm{C}-2^{\prime}$ is exceptionally mobile because of the neighboring effect due to basicity of the 3'-dimethylamino group. In compound 11, NOEs were observed between methylene protons in the benzyl group and the protons at $\mathrm{C}-10$ and 12 , respectively. Moreover, interactions between the alpha protons and $\mathrm{C}-10$, and those between the alpha protons and $\mathrm{C}-12$ were observed in the HMBC experiment. The structure of $\mathbf{1 1}$ was then confirmed. Structure of our 15-membered azalides was later proved by single crystallographic analysis of 31a crystallized by chloroform and hexane (chemistry of 31a: vide infra).

A novel lactone was continuously generated by the application of the key intermediate (12). Macrocyclization between 12 and 1,2-dimethylhydrazine hydrochloride under the same conditions for $\mathbf{1 6}$ gave 16-membered 11,12diazalide (18) which was then converted to an active form 20 as the first example. In order to provide distortion to the 15-membered azalactone, we synthesized a fused azalide (24). Macrocyclization between 12 and $p$ - 
Table 1 Antibacterial activities of 15-membered azalides (11 and $\mathbf{2 4 ) , ~ 1 6 - m e m b e r e d ~ d i a z a l i d e ~ ( 2 0 ) , ~ a n d ~ M O M ~}$

\begin{tabular}{|c|c|c|c|c|c|c|}
\hline \multirow{2}{*}{ No. } & \multirow{2}{*}{ Test organisma } & \multirow{2}{*}{ Characteristics } & \multicolumn{4}{|c|}{$(\mathrm{MIC}, \mu \mathrm{g} / \mathrm{ml})$} \\
\hline & & & 11 & 20 & 24 & MOM \\
\hline 1 & Staphylococcus aureus 209P JC-1 & standard & 0.5 & 0.5 & 0.5 & 0.25 \\
\hline 2 & S. aureus \#2 & susceptible & 1 & 1 & 1 & 1 \\
\hline 3 & S. aureus \#3 & susceptible & 1 & 0.5 & 0.5 & 0.5 \\
\hline 4 & S. aureus \#4 & ermA methylase (c) & $>128$ & $>128$ & $>128$ & $>128$ \\
\hline 5 & S. aureus \#5 & ermB methylase(i) & 1 & 0.5 & 0.5 & 0.5 \\
\hline 6 & S. aureus \#6 & ermC methylase(i) & 2 & 1 & 1 & 1 \\
\hline 7 & Enterococcus faecalis W-73 & standard & 4 & 4 & N.T. & 2 \\
\hline 8 & Klebsiella pneumoniae PCI602 & standard & $>128$ & $>128$ & N.T. & $>128$ \\
\hline 9 & Streptococcus pneumoniae DP1 Typel & standard & 0.25 & 0.25 & 0.5 & 0.13 \\
\hline 10 & S. pneumoniae \#2 & susceptible & 0.5 & 0.25 & 0.5 & 0.25 \\
\hline 11 & S. pneumoniae \#3 & ermAM methylase(c) & $>128$ & $>128$ & $>128$ & $>128$ \\
\hline 12 & S. pneumoniae \#4 & ermAM methylase(c) & $>128$ & $>128$ & $>128$ & $>128$ \\
\hline 13 & S. pneumoniae \#5 & ermAM methylase(i) & 8 & 8 & 128 & 4 \\
\hline 14 & S. pneumoniae \#6 & ermAM methylase(i) & 16 & 32 & 128 & 8 \\
\hline 15 & S. pneumoniae \#7 & mefE efflux & 0.5 & 0.25 & 0.25 & 0.25 \\
\hline 16 & S. pneumoniae \#8 & mefE efflux & 0.25 & 0.25 & 0.25 & 0.13 \\
\hline 17 & Streptococcus pyogenes Cook & standard & 0.13 & 0.13 & 0.13 & 0.13 \\
\hline 18 & S. pyogenes \#2 & ermAM methylase(c) & $>128$ & $>128$ & $>128$ & $>128$ \\
\hline 19 & S. pyogenes \#3 & mefE efflux & 0.25 & 0.25 & 0.5 & 0.13 \\
\hline 20 & Moraxella catarrhalis \#1 & susceptible & 0.5 & 0.5 & 0.5 & 0.5 \\
\hline 21 & M. catarrhalis \#2 & susceptible & 1 & 1 & 1 & 1 \\
\hline 22 & Haemophilus influenzae \#3 & susceptible & 4 & 4 & 4 & 4 \\
\hline 23 & H. influenzae \#4 & susceptible & 32 & 16 & 16 & 16 \\
\hline 24 & H. influenzae \#5 & susceptible & 32 & 16 & 16 & 16 \\
\hline
\end{tabular}

${ }^{\text {a } A l l ~ s t r a i n s ~ e x c e p t ~ s t a n d a r d ~ o r g a n i s m s ~ w e r e ~ c l i n i c a l l y ~ i s o l a t e d . ~ N . T .: ~ N o t ~ t e s t e d . ~}$

methoxybenzylamine gave methoxybenzyl azalide (21) which was consequently converted to aminodiol (22) by complete methanolysis and then hydrogenolysis in dioxaneethanol. Reaction of $\mathbf{2 2}$ with triphosgene in the presence of triethylamine in dichloromethane gave a fused azalide (23) which was finally transformed to an alternative 15membered azalide (24).

When antibacterial activities of these three prototypes were compared with those of MOM as shown in Table 1, $\mathbf{1 1}$ and $\mathbf{2 0}$ exhibited almost the same activities as those of MOM. Although we were interested in both pharmacophores of $\mathbf{1 1}$ and 20, we chose 15-membered azalide as a preliminary lead. It was impossible to isolate regioisomers when we used an unsymmetrical hydrazine, for example 1-benzyl-2-methylhydrazine, instead of a symmetrical hydrazine like 1,2-dimethylhydrazine, and we recognized that further chemical modification of 16membered diazalide (20) would not be efficient. Moreover, deprotection of 11,12-diazalide prepared by $\mathbf{1 2}$ with unsubstituted hydrazine gave complicated results. Then we analyzed relationships between the methylene length in the spacer and antimicrobial activities in 15-membered azalides.

\section{Relationships between Spacer Length and Activities}

Cyclization of $\mathbf{1 2}$ with methylamine gave the simplest framework in this series, 25, as shown in Scheme 2, which was consequently converted to $\mathbf{2 7}$. Methanolysis at the C-2' position of $\mathbf{2 5}$ involves deacetylation at C-9 because of low steric hindrance of the $\mathrm{N}$-methyl group at the 11-position. Next, a variety of phenylalkyl derivatives of 15 -membered azalides were synthesized. Compounds $\mathbf{2 8 a} \sim \mathbf{2 8 d}$ were respectively prepared in approximately $10 \%$ yield from $\mathbf{1 2}$. Methanolysis of $\mathbf{2 8 a} \sim \mathbf{d}$ gave $2^{\prime}$-hydroxyl derivatives $(29 \mathbf{a} \sim 29 \mathbf{c})$ and 9,2'-dihydroxyl derivatives $(30 \mathbf{3} \sim \mathbf{3 0 d})$, which were converted into desired 31a $\sim 31 \mathbf{c}$ and 32a $\sim 32 d$, respectively, by treatment with difluoroacetic acid.

As shown in Table 2, antibacterial activities of 9- 


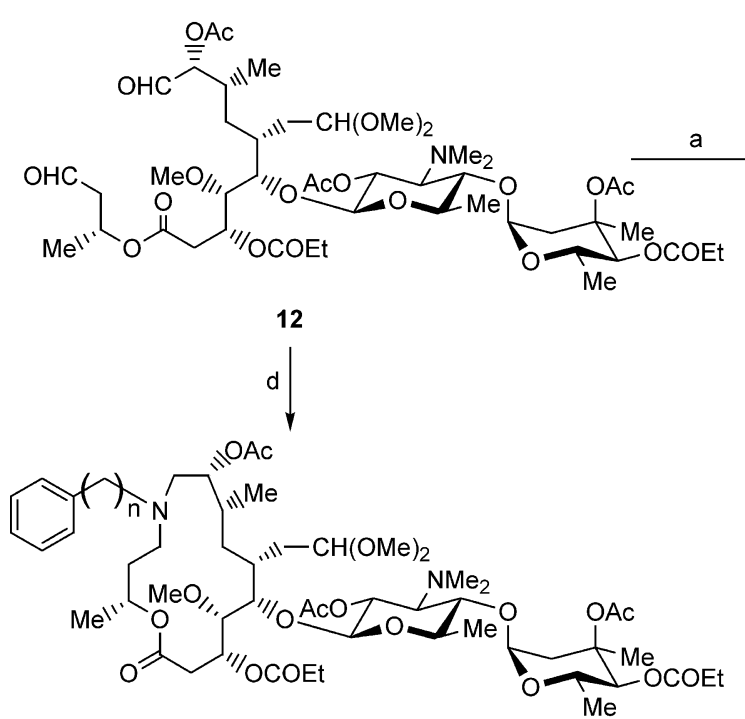

28a: $n=2,28 b: n=3,28 c: n=4,28 d: n=5$

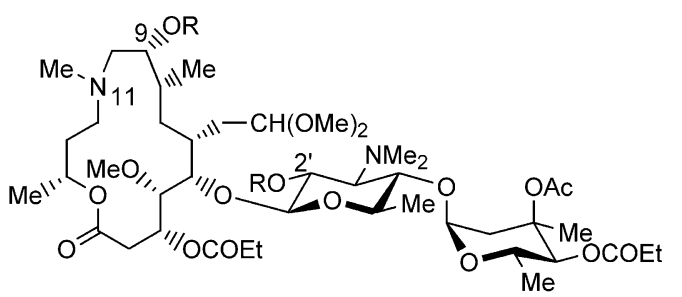

$\mathrm{b} \longrightarrow$ 25: $\mathrm{R}=\mathrm{Ac}$
26: $\mathrm{R}=\mathrm{H}$

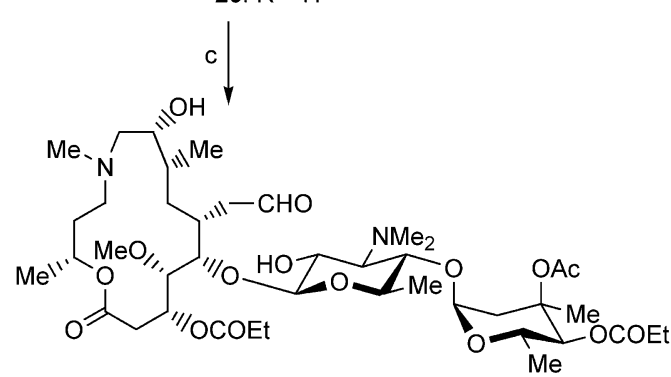

27

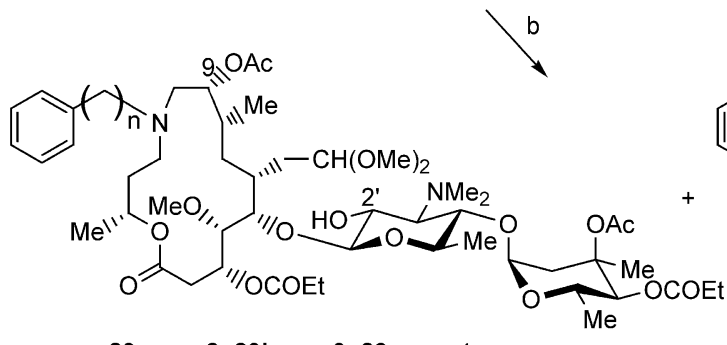

29a: $n=2,29 b: n=3,29 c: n=4$

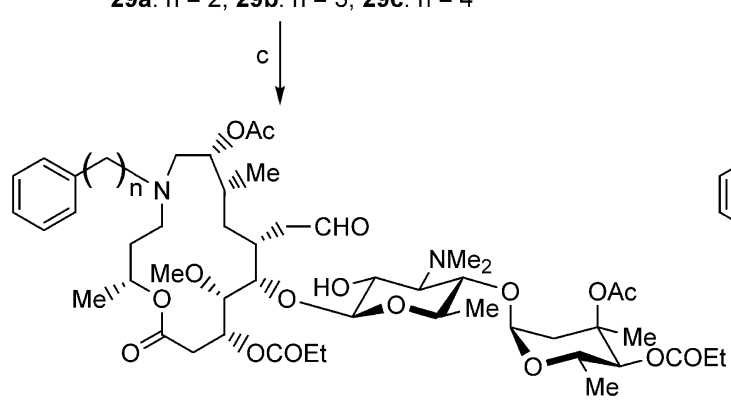

31a: $n=2,31 b: n=3,31 c: n=4$

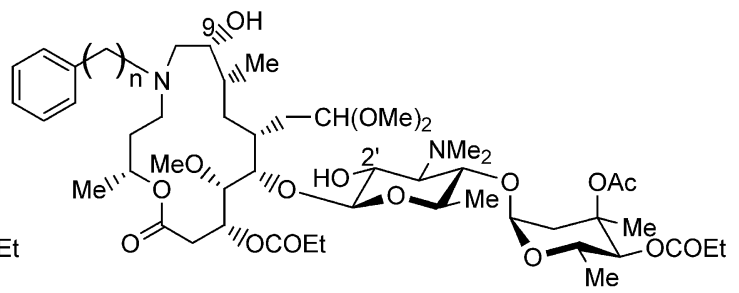

30a: $n=2,30 b: n=3,30 c: n=4,30 d: n=5$

c

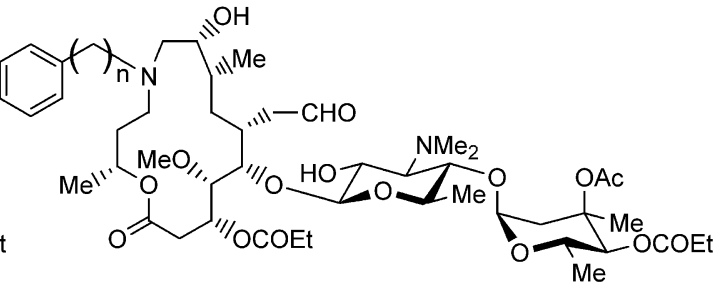

32a: $n=2,32 b: n=3,32 c: n=4,32 d: n=5$

Reagents and conditions: (a): methylamine hydrochloride (1.1 eq), $\mathrm{NaB}(\mathrm{CN}) \mathrm{H}_{3}(3.9 \mathrm{eq}), \mathrm{AcOH}(15 \mathrm{eq}), \mathrm{EtOH}, 0^{\circ} \mathrm{C}$ to r.t., 16 hours, $11 \%$; (b): $\mathbf{M e O H}$, r.t., 72 hours, 59\% for $\mathbf{2 6}$, see experimental for $\mathbf{2 9}$ and $\mathbf{3 0}$, As an exception, 29c and $\mathbf{3 0 c}$ were separately prepared from $\mathbf{2 8 c}$; (c): difluoroacetic acid (20 eq), $\mathrm{MeCN}-\mathrm{H}_{2} \mathrm{O}$, r.t., 24 hours, $70 \%$ for 27, see experimental for $\mathbf{3 1}$ and 32; (d): 2-phenylethylamine (1.1 eq), $\mathrm{NaB}(\mathrm{CN}) \mathrm{H}_{3}$ (3.9 eq), $\mathrm{AcOH}(15 \mathrm{eq}), \mathrm{EtOH}, 0^{\circ} \mathrm{C}$ to r.t., 16 hours, $7.3 \%$ for $\mathbf{2 8 a}$, see experimental for $\mathbf{2 8 b} \sim \mathbf{2 8 d}$.

Scheme 2 Synthesis of $N$-phenylalkyl-azalides with a variety of spacer length.

hydroxyl analogues 32a $\sim$ 32d are generally stronger than those of 9-acetoxy analogues 31a 31c. In addition, it was found that the spacer length was optimized to $\mathrm{C}_{3}$ or $\mathrm{C}_{4}$ among these phenylalkyl analogues. We thus decided to confirm an appropriate template for further medicinal chemistry before fundamental optimization of 15-membered 11-azalide.

\section{Appropriate Template and Fundamental Optimization}

At this stage, we had to confirm antibacterial activities of 15-membered azalide which did not possess a neutral sugar, 41, as shown in Scheme 3. In general, a neutral sugar enhances its antibacterial activities and sometimes plays an important role to exhibit activities against resistant bacteria as several examples reported [26]. But recent ketolides do not have a neutral sugar, so we had to prepare 15 membered azalide without a neutral sugar and measure 


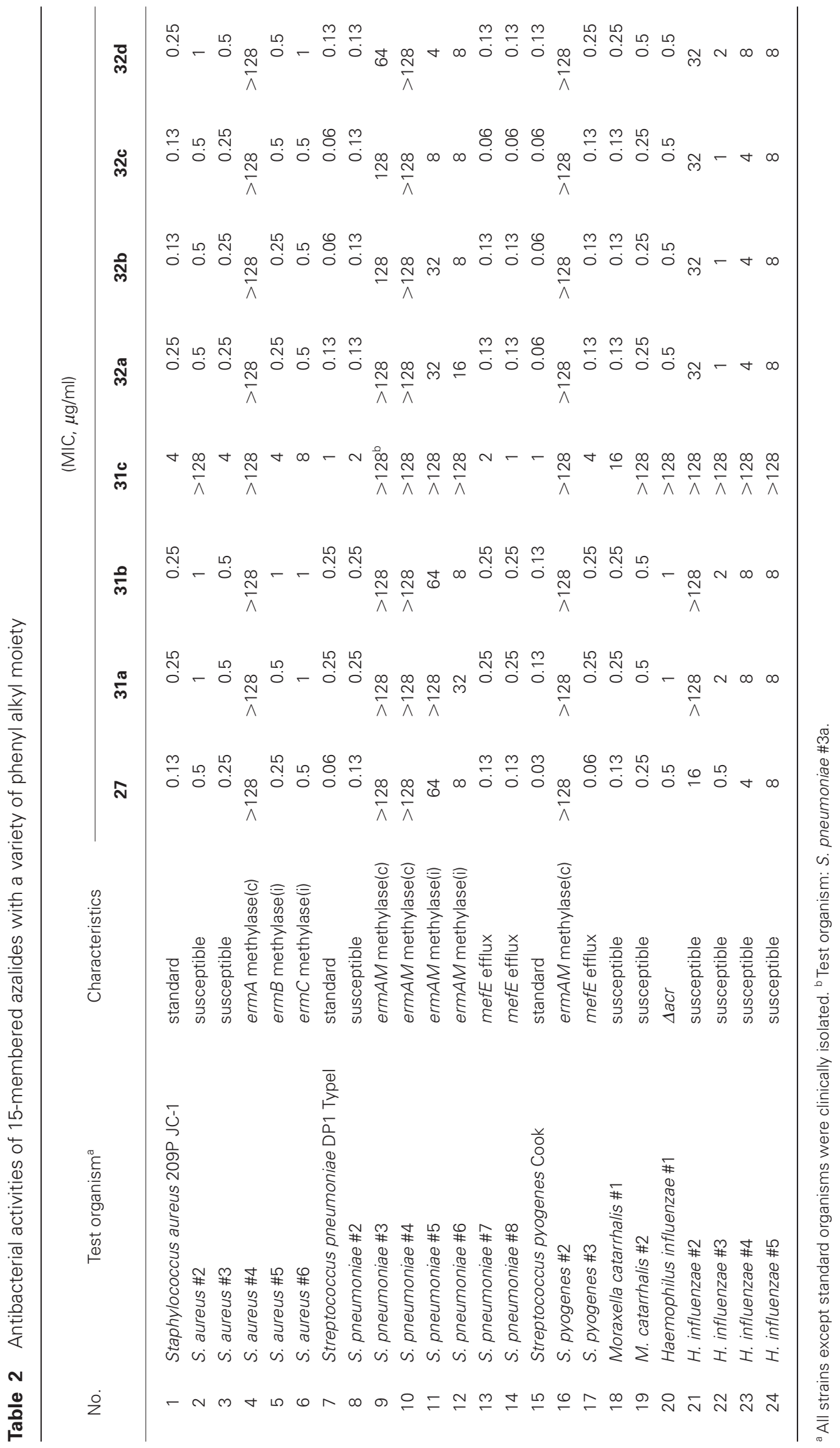




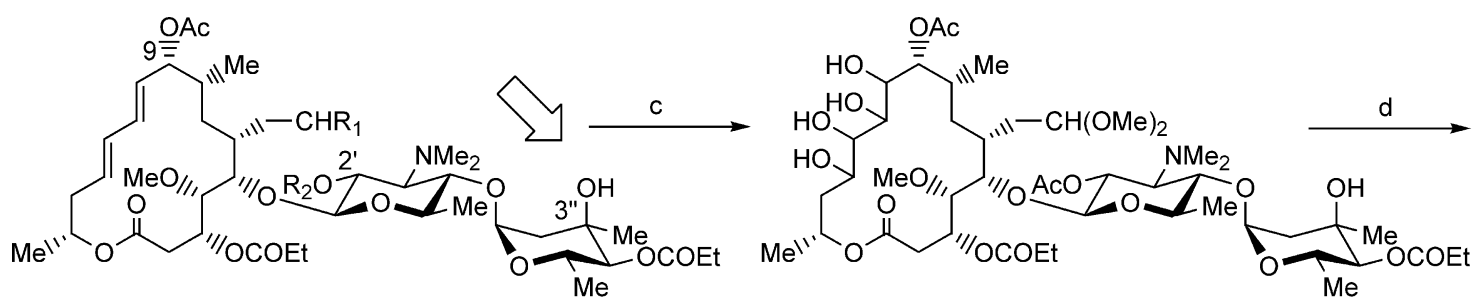

36

a 33: $R_{1}=O, R_{2}=H$
$b \longrightarrow 34: R_{1}=(O M e)_{2}, R_{2}=H$
$b 35: R_{1}=(O M e)_{2}, R_{2}=A c$

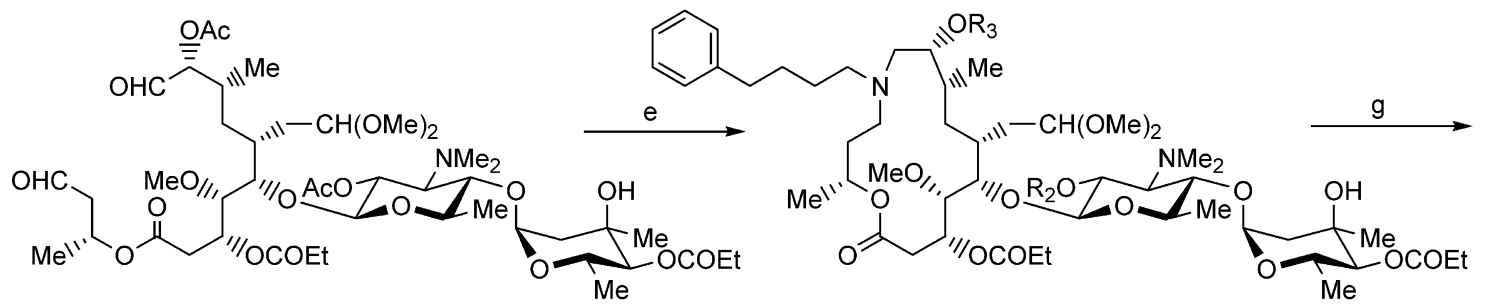

37

f 38: $R_{2}=R_{3}=A c$

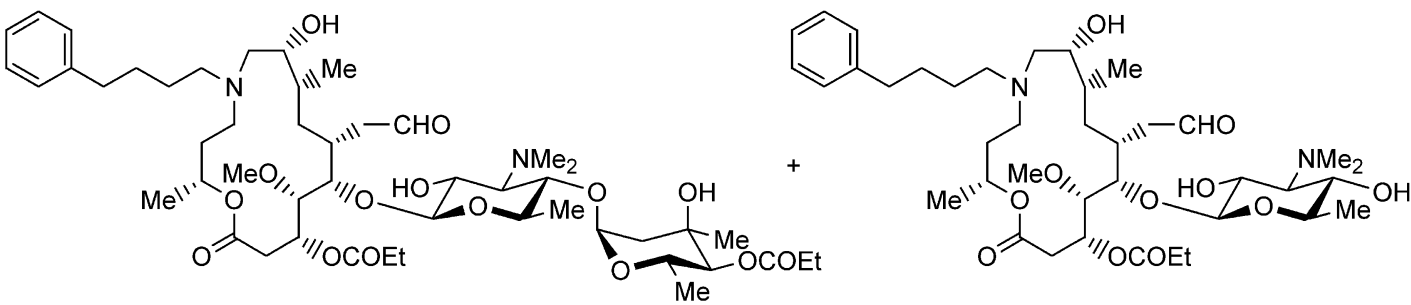

40

41

Reagents and conditions: (a): $(\mathrm{MeO})_{3} \mathrm{CH}(80 \mathrm{eq}), \mathrm{PPTS}(1.2 \mathrm{eq}), \mathrm{MeOH}, 40 \sim 50^{\circ} \mathrm{C}, 4$ days; (b): $\mathrm{Ac}_{2} \mathrm{O}\left(5\right.$ eq), $\mathrm{MeCN}, 40^{\circ} \mathrm{C}, 16 \mathrm{hours}$; (c): $\mathrm{OsO}_{4}(0.15 \mathrm{eq})$, $\mathrm{NMO}(2 \mathrm{eq})$, aq. acetone, r.t., 24 hours, $41 \%$ for $\mathbf{3 6}, 30 \%$ in 3 steps based on $\mathbf{3 3}, 34 \%$ for $\mathbf{4 5}$; (d): $\mathrm{Pb}(\mathrm{OAc})_{4}(2.1 \mathrm{eq}), \mathrm{PhH}$, $\mathrm{Na}_{2} \mathrm{CO}_{3}$ (8 eq), r.t., 10 minutes; (e): 4-phenylbutylamine (1.1 eq), $\mathrm{NaB}(\mathrm{CN}) \mathrm{H}_{3}\left(3.9\right.$ eq), $\mathrm{AcOH}(15 \mathrm{eq}), \mathrm{EtOH}, 0^{\circ} \mathrm{C}$ to r.t., $15 \sim 20$ hours, $8.4 \%$ for $\mathbf{3 8}$ in 2 steps, $9.8 \%$ for $\mathbf{4 7}$ in 2 steps; (f): $\mathbf{M e O H}$, r.t., 48 hours, $88 \%$ for $\mathbf{3 9}$ and $74 \%$ for $\mathbf{4 8}$, (g): difluoroacetic acid (20 eq), $\mathrm{MeCN}-\mathrm{H}_{2} \mathrm{O}$, r.t., 24 hours, $37 \%$ for $\mathbf{4 0}$ and $\mathbf{4 3 \%}$ for $\mathbf{4 1}, 98 \%$ for $\mathbf{4 9}$.

Scheme 3 Synthesis of monosaccharide (41) and rokitamycin-type azalide (50).

its biological activities. As we previously mentioned in this article, a glycosidic bond at the neutral sugar can be cleaved under acidic conditions, if the C- 3 " position is a free hydroxyl group. Thus, we used 9-Oacetylmidecamycin $\mathrm{A}_{1}$ (33) [27] possessing a hydroxyl group at the C-3" position as a starting material. 33 was sequentially converted to a key intermediate, dialdehyde (37) which was macrocyclized with 4-phenylbutylamine to afford 38. Complete methanolysis of $\mathbf{3 8}$ followed by treatment with difluoroacetic acid gave monosaccharide (41) accompanied with disaccharide (40).

Generally speaking, antibacterial activities of a 3hydroxyl derivative tend to be stronger than those of corresponding 3-acyloxy analogue in 16-membered macrolides. We therefore designed a synthetic route for the 3-hydroxyl analogue of 15-membered azalide (50) as shown in Scheme 3. We usually apply the 3,18-O-silylhemiacetal protection $[7 \mathrm{~b}, 28,29]$ for compounds which possess a free aldehyde group at C-18 and a free hydroxyl group at C-3. We decided, however, to apply dimethyl acetal protection at $\mathrm{C}-18$ and no protection at $\mathrm{C}-3$, because there seemed to be interference by a seven-membered ring of 3,18-O-silyl-hemiacetal toward the formation of 15 membered azalide. Sequentially protected rokitamycin [26] (44) [22b, 32] was oxidized to give a key intermediate (46), which was cyclized and then sequentially deprotected to afford the 3-hydroxyl analogue (50).

Preliminarily expected, $\mathbf{4 1}$ did not exhibit strong antibacterial activities as shown in Table 3. On the other hand, 50 showed strong activities, but there was not any big improvement compared to 32c in spite of more reaction steps required. Then we performed fundamental 


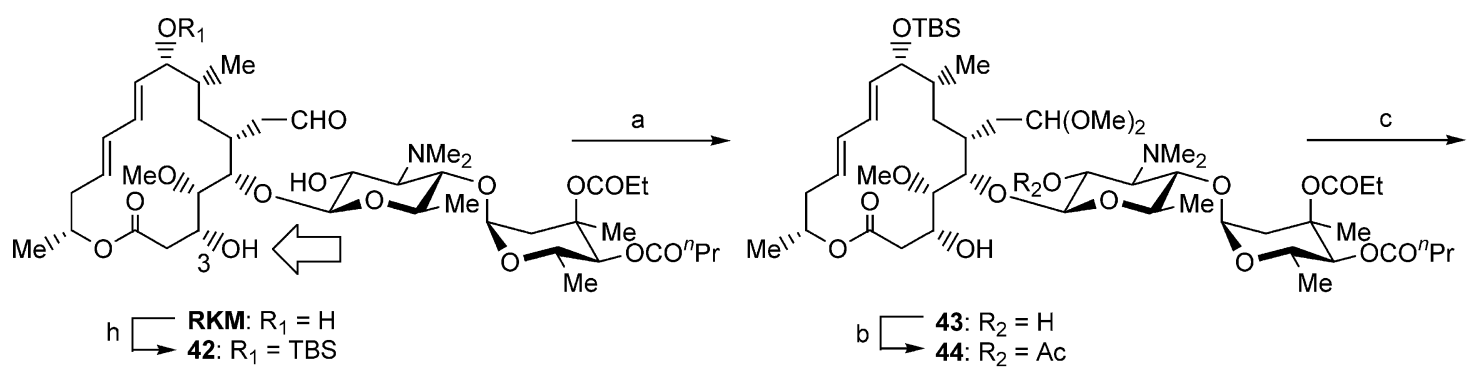

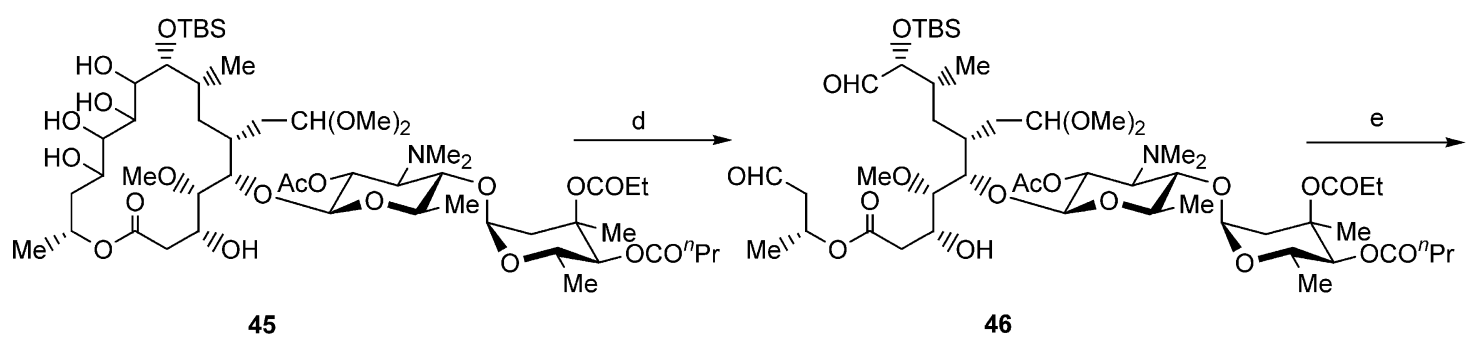

45

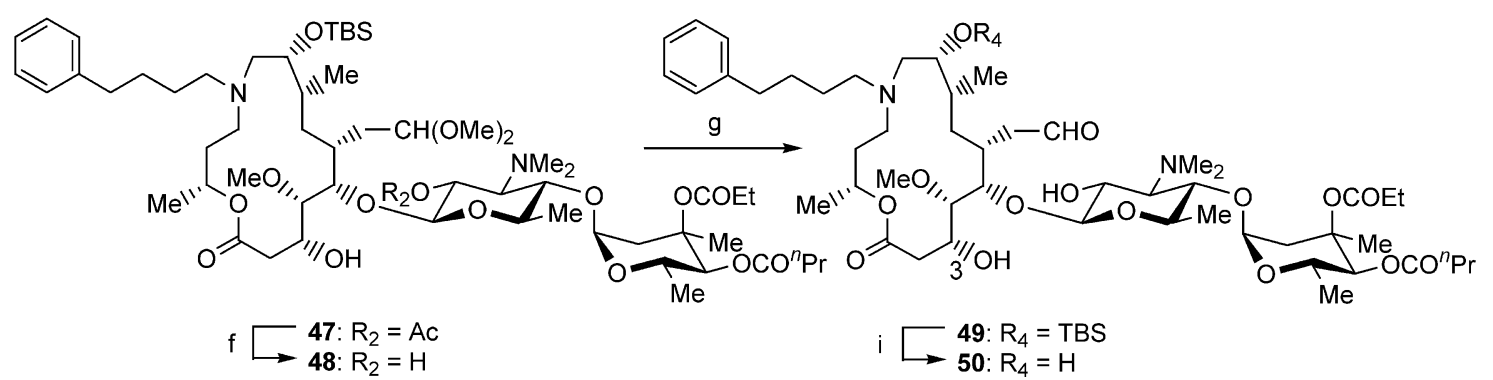

Reagents and conditions: (h): 1) t-butyldimethylsilyl chloride (1.5 eq), imidazole (3.3 eq), DMF, $45^{\circ} \mathrm{C}, 17$ hours, 2 ) TBAF (1.4 eq), THF, r.t, 3 hours, $90 \%$; (i): $\operatorname{TBAF}(5 \mathrm{eq}), \mathrm{AcOH}-\operatorname{THF}(1: 1), 60^{\circ} \mathrm{C}, 48$ hours, $54 \%$.

Scheme 3 Synthesis of monosaccharide (41) and rokitamycin-type azalide (50). (continued)

optimization of 15 -membered azalide focusing on the chemical structure of $\mathbf{3 2} \mathbf{b}$ as a lead compound as shown in Fig. 5. Synthesis of $\mathbf{5 2}$ was performed as in the case of 32b. Compound 54, an alternative hydrazine-based 15membered azalide, was prepared via macrocyclization of 12 and 1-methyl-1-(3-phenylpropyl)hydrazine. The acetoxy group at the C-9 position was, however, unexpectedly reduced in the course of macrocyclization. Syntheses of $\mathbf{5 1}, \mathbf{5 3}$, and $\mathbf{5 5}$ to $\mathbf{5 8}$ were completed by $N$-alkylation of $\mathbf{2 2}$ (Scheme 1) or $N$-acylation (for 57) and deprotection. Among these partially optimized derivatives, quinoline analogues with a saturated alkyl chain (52 and 53) exhibited the strongest antibacterial activities in this series as shown in Table 4, and were especially effective against resistant bacteria of $S$. pneumoniae which had the erm gene controlling inducible methylation of bacterial ribosome. Moreover, it is notable that these novel azalides are almost not affected by efflux pump function in S. pneumoniae.

\section{Sustainable Chemistry for Synthesis of Azalides}

So far we described the synthesis of novel 15-membered azalides starting from 16-memberd macrolides, leucomycin analogues including MOM, MDM, and rokitamycin (RKM). We, however, used osmium tetroxide and lead (IV) acetate in order to synthesize the key intermediates, dialdehydes $(\mathbf{1 2}, \mathbf{3 7}$, and 46$)$. We have to pay attention to human health and environmental preservation when we use these reagents in a large scale.

Thus, we applied direct oxidation method using ozone to afford the dialdehyde from the diene, $\mathbf{1 4}$ or $\mathbf{1 5}$. Because the dialdehyde was very mobile as we have already mentioned, we confirmed the completion of this approach by detection of 30c [30] as shown in Scheme 4. 30c prepared with this ozone route was fully identified with that synthesized by the original route shown in Scheme 2 by FAB-MS and ${ }^{1} \mathrm{H}-$ NMR. As a result, we could omit the isolation process of the tetraol. In addition, we could reduce the number of total 
Table 3 Antibacterial activities of 15-membered azalides with a variety of template

\begin{tabular}{|c|c|c|c|c|c|c|c|}
\hline \multirow{2}{*}{ No. } & \multirow{2}{*}{ Test organismª } & \multirow{2}{*}{ Characteristics } & \multicolumn{5}{|c|}{ (MIC, $\mu \mathrm{g} / \mathrm{ml})$} \\
\hline & & & 40 & 41 & MDM & 50 & RKM \\
\hline 1 & Staphylococcus aureus 209P JC-1 & standard & 0.25 & 1 & 0.25 & 0.25 & 0.06 \\
\hline 2 & S. aureus \#2 & susceptible & 0.5 & 2 & 0.5 & 0.5 & 0.25 \\
\hline 3 & S. aureus \#3 & susceptible & 0.25 & 1 & 0.25 & 0.25 & 0.13 \\
\hline 4 & S. aureus \#4 & ermA methylase(c) & $>128$ & $>128$ & $>128$ & $>128$ & $>128$ \\
\hline 5 & S. aureus \#5 & ermB methylase(i) & 0.25 & 2 & 0.25 & 0.25 & 0.13 \\
\hline 6 & S. aureus \#6 & ermC methylase(i) & 0.5 & 2 & 0.5 & 0.5 & 0.25 \\
\hline 7 & $\begin{array}{l}\text { Streptococcus pneumoniae DP1 } \\
\text { Typel }\end{array}$ & standard & 0.06 & 0.5 & 0.06 & 0.06 & 0.03 \\
\hline 8 & S. pneumoniae \#2 & susceptible & 0.13 & 0.5 & 0.13 & 0.06 & 0.03 \\
\hline 9 & S. pneumoniae \#3a & ermAM methylase $(c)+$ mefE & $>128$ & $>128$ & $>128$ & $>128$ & $>128$ \\
\hline 10 & S. pneumoniae \#4 & ermAM methylase(c) & $>128$ & $>128$ & $>128$ & 64 & $>128$ \\
\hline 11 & S. pneumoniae \#6 & ermAM methylase(i) & 32 & $>128$ & 128 & 1 & 0.5 \\
\hline 12 & S. pneumoniae \#7 & mefE efflux & 0.06 & 0.5 & 0.06 & 0.06 & 0.03 \\
\hline 13 & S. pneumoniae \#8 & mefE efflux & 0.06 & 0.5 & 0.13 & 0.13 & 0.06 \\
\hline 14 & Streptococcus pyogenes Cook & standard & N.T. & N.T. & N.T. & N.T. & N.T. \\
\hline 15 & S. pyogenes \#2 & ermAM methylase(c) & N.T. & N.T. & N.T. & N.T. & N.T. \\
\hline 16 & S. pyogenes \#3 & mefE efflux & 0.13 & 1 & 0.13 & 0.06 & 0.03 \\
\hline 17 & Moraxella catarrhalis \#1 & susceptible & 0.5 & 2 & 2 & 0.25 & 0.06 \\
\hline 18 & M. catarrhalis \#2 & susceptible & 0.5 & 2 & 2 & 0.25 & 0.13 \\
\hline 19 & Haemophilus influenzae \#1 & $\Delta a c r$ & 0.5 & 1 & 0.5 & 0.5 & 0.25 \\
\hline 20 & H. influenzae \#2 & susceptible & 32 & 64 & 32 & 16 & 8 \\
\hline 21 & H. influenzae \#3 & susceptible & 1 & 8 & 2 & 1 & 1 \\
\hline 22 & H. influenzae \#4 & susceptible & 8 & 32 & 16 & 4 & 4 \\
\hline 23 & H. influenzae \#5 & susceptible & 16 & 128 & 16 & 8 & 4 \\
\hline
\end{tabular}

${ }^{\text {a }}$ All strains except standard organisms were clinically isolated. N.T.: Not tested.

reaction steps, since this methodology did not require protection of the 2 '-hydroxyl group. Our original synthetic route required five steps for $30 \mathrm{c}$ based on 14 via $15,13,12$ and 28c, and a five-step yield was $0.43 \%$. However, this ozone route can provide 30c in $8.0 \%$ based on $\mathbf{1 4}$ in two steps. On the other hand, ozone oxidation can be applicable in "ton scale", [31] so these preliminary results regarding sustainable chemistry might be an acceptable solution for further experiments focusing on our azalide chemistry.

\section{Conclusions}

Novel 15-membered 11-azalides and 16-membered 11,12diazalide starting from 16-membered macrolides were designed and synthesized. 12 was isolated via $\mathbf{1 3}$ which was prepared by osmium oxidation of a conjugated diene. One-pot macrocyclization of $\mathbf{1 2}$ with benzylamine or 1,2-dimethylhydrazine followed by deprotections afforded corresponding $\mathbf{1 1}$ or $\mathbf{2 0}$, although the reaction conditions could not be optimized. When we used an unsymmetrical hydrazine as a diamine for macrocyclization with the dialdehyde, it was impossible to readily isolate regioisomers. We thus focused on chemical modification of 15 -membered azalide.

In optimization of the spacer length, it became clear that $\mathrm{C}_{3}$ to $\mathrm{C}_{4}$ methylene exhibited strong antibacterial activities. As for a template for further medicinal chemistry, we chose the MOM framework which possessed 3"-O-acetyl-3-Opropionyl-pharmacophore. As a result of fundamental optimization of an aryl moiety at the 11-position, $\mathbf{5 2}$ and $\mathbf{5 3}$ exhibited the strongest antibacterial activities in this series, and were especially effective against resistant bacteria of $S$. pneumoniae which had the erm gene controlled inducible methylation of bacterial ribosome. Fifteen-membered 11azalides disclosed their potentiality as a lead molecule for further drug discovery research.

For environmental preservation, the sustainable chemistry 

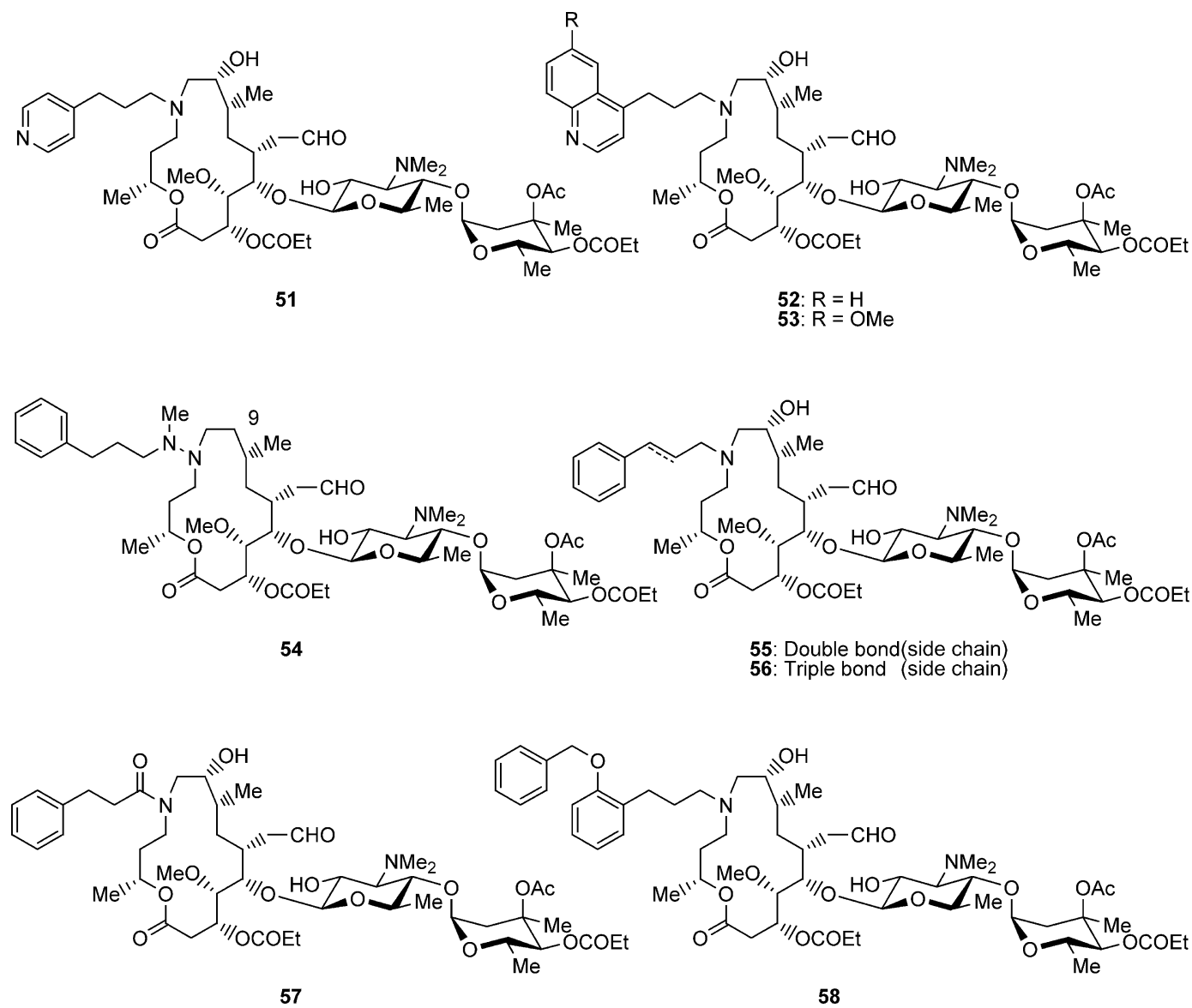

Fig. 5 Fundamental optimization of 15-membered azalides.

in application of ozone oxidation for synthesis of the key intermediate, dialdehyde, was also introduced as a preliminary solution for process chemistry of 15 -membered 11-azalides. This approach practically decreased reaction steps and remarkably improved the synthetic yield.

\section{Experimental}

\section{General Methods}

Optical rotations were measured on a Perkin-Elmer 241 Polarimeter or Jasco P-1030 Polarimeter. Fast-atom bombardment (FAB) mass spectra were recorded on a JEOL JMS-700 instrument. ${ }^{1} \mathrm{H}-\mathrm{NMR}$ spectra were recorded on Varian Gemini-300 spectrometers with chemical shifts reported in ppm relative to internal tetramethylsilane. ${ }^{13} \mathrm{C}-\mathrm{NMR}$ spectra were measured with a Jeol JNMGSX 400 NMR spectrometer for $100 \mathrm{MHz}$. Silica gel chromatography and preparative TLC were performed on Waco C-200 or C-300 and Merck TLC $60 \mathrm{~F}_{254}$ Art. 5744, respectively and visualized with a UV lamp or $10 \% \mathrm{H}_{2} \mathrm{SO}_{4}$ containing $2.0 \%$ sodium molybdate and $2.0 \%$ phosphoric acid. Evaporation was carried out under reduced pressure below $35^{\circ} \mathrm{C}$, unless otherwise noted.

9,2',3"-Tri- $O$-acetyl-10,11,12,13-tetrahydro-10,11,12,13tetrahydroxymidecamycin $\mathrm{A}_{1}$ 18-dimethylacetal (13)

To a solution of 9,3"-di-O-acetylmidecamycin $\mathrm{A}_{1}$ 18dimethylacetal (14) [8b] $(64.2 \mathrm{~g})$ in acetonitrile $(610 \mathrm{ml})$ was added acetic anhydride $(7.8 \mathrm{ml})$, and the mixture was stirred at $40^{\circ} \mathrm{C}$ for 16 hours. After the reaction mixture was concentrated under reduced pressure, ethyl acetate $(660 \mathrm{ml})$ was added, and the organic layer was successively washed twice with saturated aqueous sodium hydrogencarbonate solution $(300 \mathrm{ml})$, and saturated brine $(300 \mathrm{ml})$. The organic layer was dried over anhydrous sodium sulfate, and then filtered, and the filtrate was concentrated under reduced pressure to obtain $9,2^{\prime}, 3^{\prime \prime}$-tri- $O$-acetylmidecamycin $\mathrm{A}_{1} 18$ dimethylacetal (15) (67.0 g).

To a solution of $\mathbf{1 5}(20.0 \mathrm{~g})$ in acetone $(500 \mathrm{ml})$ and water $(77 \mathrm{ml}), N$-methylmorpholine- $N$-oxide $(9.5 \mathrm{ml})$ and $4.0 \%$ aqueous osmium tetraoxide $(19.5 \mathrm{ml})$ were added and the mixture was stirred at room temperature. After 20 hours, $N$-methylmorpholine- $N$-oxide $(2.4 \mathrm{ml})$ was added, 


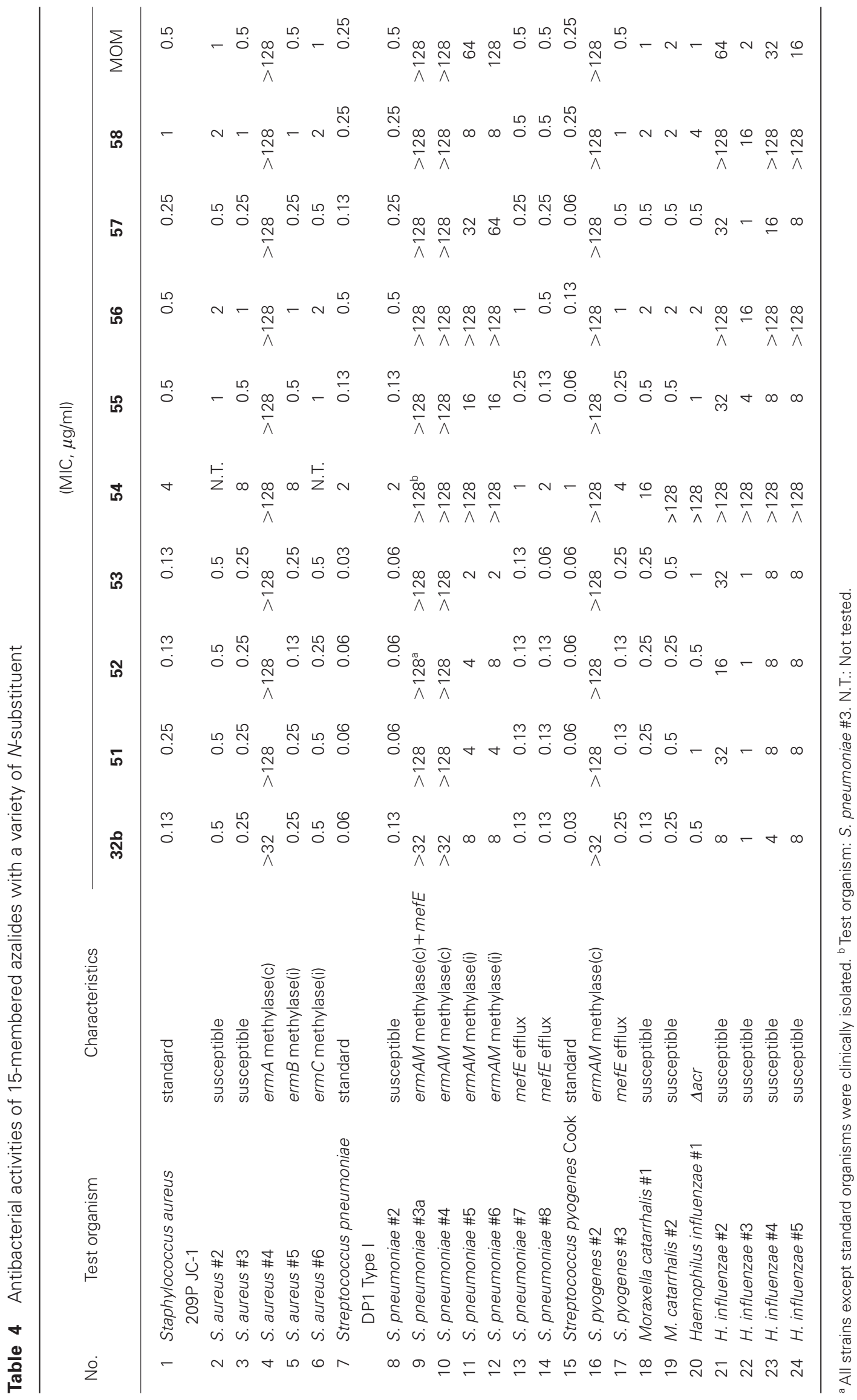



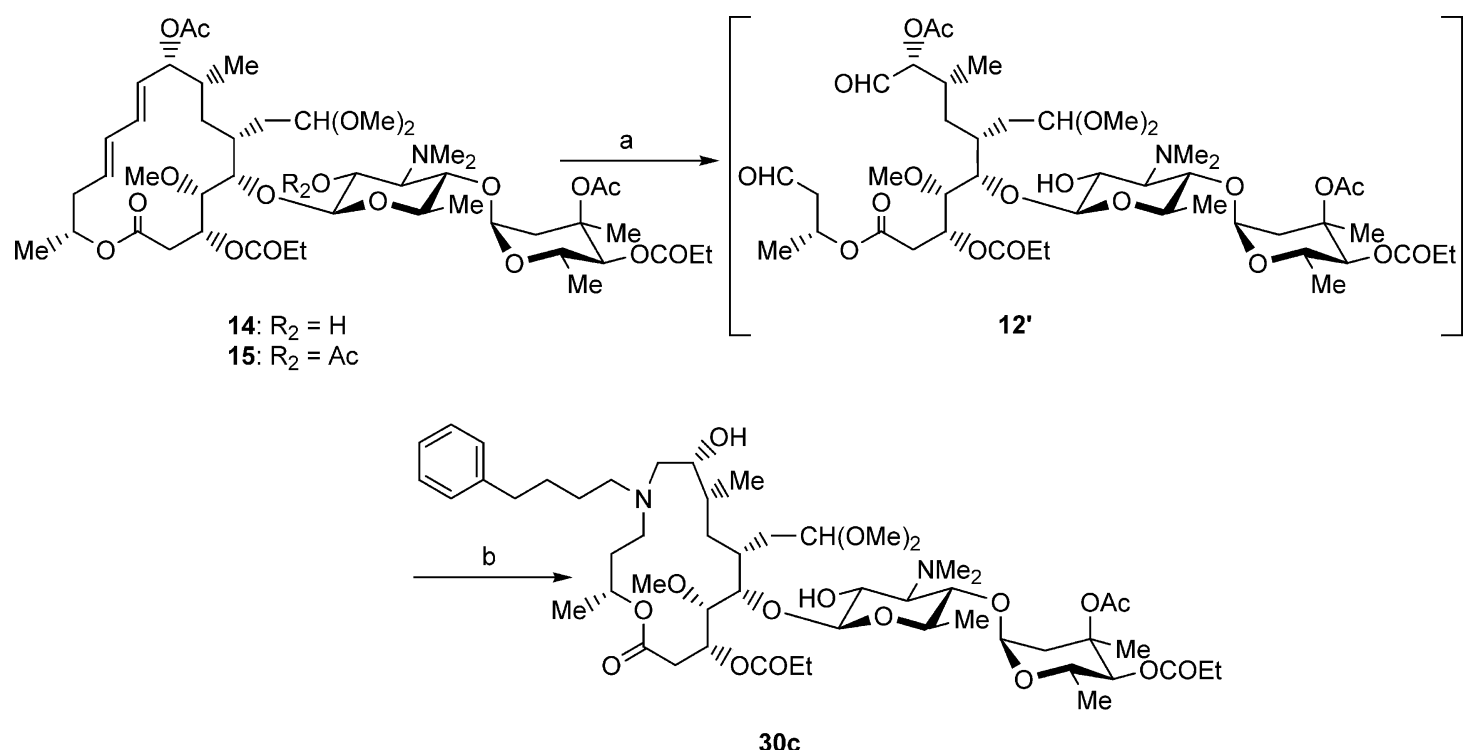

Reagents and conditions: (a): $\mathrm{O}_{3}$, abs. $\mathrm{MeOH},-78^{\circ} \mathrm{C}, 15$ minutes, then, $\mathrm{O}_{2}$ bubbling for $5 \sim 10$ minutes, $\mathrm{Me}_{2} \mathrm{~S},-78^{\circ} \mathrm{C}, 30$ minutes; $(\mathrm{b})$ : 4-phenylbutylamine (1.1 eq), $\mathrm{NaB}(\mathrm{OAc})_{3} \mathrm{H}(3.0 \mathrm{eq}), \mathrm{AcOH}$, r.t. Two-step yield is $8.0 \%$ from 14 to $30 \mathrm{c}$.

Scheme 4 Sustainable chemistry for construction of 15-membered azalide.

and the mixture was further stirred for 4 hours. After the reaction mixture was concentrated under reduced pressure, ethyl acetate $(600 \mathrm{ml})$ was added, and the organic layer was successively washed with water $(200 \mathrm{ml}), 5.0 \%$ aqueous sodium thiosulfate solution $(300 \mathrm{ml})$ and saturated brine $(300 \mathrm{ml})$. The organic layer was dried over anhydrous sodium sulfate, and filtered, and then the filtrate was concentrated under reduced pressure. The resulting residue was roughly purified by flash silica gel column chromatography (chloroform/methanol $(25: 1 \sim 15: 1)$ ) and further purified by flash silica gel column chromatography (chloroform/ethyl acetate/methanol $(30: 30: 1 \sim 25: 25: 1)$ ) to obtain 13 ( $8.46 \mathrm{~g}, 40 \%$ based on 14 ) as a colorless solid.

In the case of sequential reactions without purification of 14, three-step yield of $\mathbf{1 3}$ based on MOM was $30 \%$.

13: $[\alpha]_{\mathrm{D}}^{21}-81^{\circ}\left(c \quad 1.0, \mathrm{CHCl}_{3}\right)$; FAB-MS $m / z 1054$ $(\mathrm{M}+\mathrm{H})^{+}$as $\mathrm{C}_{49} \mathrm{H}_{83} \mathrm{NO}_{23} ;{ }^{1} \mathrm{H}-\mathrm{NMR}\left(300 \mathrm{MHz}, \mathrm{CDCl}_{3}\right) \delta$ : 0.96 (d, 19-H), 1.07 (d, 6"-H), 1.13 (t, 3-OCOCH $\mathrm{CH}_{3}$ ), 1.19 (t, 4"-OCOCH$\left.{ }_{2} \mathrm{CH}_{3}\right), 1.20$ (d, 6'-H), 1.27 (d, 16-H), 1.41 (s, 3"- $\left.\mathrm{CH}_{3}\right), 1.50$ (br dd, 14-H), 1.68 (dd, 2"-Hax), 1.88 (br dd, 17-H), 2.03 (s, 9-OCOCH ${ }_{3}$ ), 2.04 (s, 3"--OCOCH ${ }_{3}$ ), $2.17\left(\mathrm{~s}, 2^{\prime}-\mathrm{OCOCH}_{3}\right), 2.35(\mathrm{~m}, 8-\mathrm{H}), 2.44\left(\mathrm{~s}, 3^{\prime}-\mathrm{N}\left(\mathrm{CH}_{3}\right)_{2}\right)$, 2.60 (t, 3'-H), 2.73 (dd, 2-H), 3.15 (t, 4'-H), 3.18 (s, 18$\left.\mathrm{OCH}_{3}\right), 3.20$ (d, 2"-Heq), $3.22\left(\mathrm{~s}, 18-\mathrm{OCH}_{3}\right), 3.27$ (dq, 5'$\mathrm{H}), 3.39$ ( br d, 4-H), 3.57 (s, 4- $\left.\mathrm{OCH}_{3}\right), 3.63(\mathrm{~m}, 12-\mathrm{H}), 3.83$ (br d, 5-H), 3.91 (dd, 10-H), 4.09 (brt, 13-H), 4.38 (dd, 18H), 4.48 (dq, 5"-H), 4.57 (d, 4"-H), 4.70 (d, 1'-H), 4.82 (d, $\left.1^{\prime \prime}-\mathrm{H}\right), 4.96$ (dd, 2'-H), $5.02(\mathrm{~m}, 9-\mathrm{H}), 5.04(\mathrm{~m}, 15-\mathrm{H}), 5.34$ (br d, 3-H).
(-)-(1R)-1-Methyl-3-oxopropyl( $3 R, 4 S, 5 S, 6 R, 8 R, 9 R)$-9acetoxy-5-[2- $O$-acetyl-4- $O-(3-O$-acetyl-2,6-dideoxy-3methyl-4- $O$-propionyl- $\alpha$-L-ribo-hexopyranosyl)-3,6dideoxy-3-dimethylamino- $\beta$-D-glucopyranosyloxy]-6-(2,2dimethoxyethyl)-4-methoxy-8-methyl-10-oxo-3-propionyloxydecanoate (12)

To a solution of $\mathbf{1 3}(30 \mathrm{mg})$ in benzene $(1.0 \mathrm{ml})$ was added sodium carbonate $(18 \mathrm{mg})$, and then lead tetraacetate $(29 \mathrm{mg})$ divided into 5 portions were added over 20 minutes. After the reaction mixture was stirred at room temperature for 1 hour, the supernatant was transferred into a separatory funnel. To the residue was added benzene $(5.0 \mathrm{ml})$, and the supernatant was transferred into the separatory funnel, and then the same operations were repeated three times. Water $(10 \mathrm{ml})$ and saturated aqueous sodium hydrogencarbonate solution $(15 \mathrm{ml})$ were added to the separatory funnel to wash the organic layer. The organic layer was further washed with saturated brine $(15 \mathrm{ml})$, dried over anhydrous sodium sulfate, and then filtered. The filtrate was concentrated under reduced pressure, and the resulting residue was purified by preparative TLC (hexane/acetone $(2: 3))$ to obtain $12(6.5 \mathrm{mg}, 23 \%)$ as a colorless solid.

12: FAB-MS $m / z 992(\mathrm{M}+\mathrm{H})^{+}$as $\mathrm{C}_{47} \mathrm{H}_{77} \mathrm{NO}_{21} ;{ }^{1} \mathrm{H}-\mathrm{NMR}$ $\left(300 \mathrm{MHz}, \mathrm{CDCl}_{3}\right) \delta: 1.05\left(\mathrm{~d}, 8-\mathrm{CH}_{3}\right), 1.08\left(\mathrm{~d}, 6^{\prime \prime}-\mathrm{H}\right), 1.14$ (t, 3-OCOCH $\mathrm{CH}_{3}$ ), 1.20 (t, 4"-OCOCH $\mathrm{CH}_{3}$ ), 1.21 (d, 6'$\mathrm{H}), 1.33\left(\mathrm{~d}, \mathrm{OCH}\left(\mathrm{CH}_{3}\right) \mathrm{CH}_{2} \mathrm{CHO}\right), 1.42\left(\mathrm{~s}, 3^{\prime \prime}-\mathrm{CH}_{3}\right), 1.68$ (dd, 2"-Hax), 1.85 (m, 6-H), 2.03 (s, 3"-OCOCH ${ }_{3}$ ), 2.07 (s, $\left.2^{\prime}-\mathrm{OCOCH}_{3}\right), 2.20\left(\mathrm{~s}, 9-\mathrm{OCOCH}_{3}\right), 2.36,2.37\left(2 \times \mathrm{q}, 4^{\prime \prime}\right.$ - 
$\left.\mathrm{OCOCH}_{2} \mathrm{CH}_{3}\right), 2.44\left(\mathrm{~s}, 3^{\prime}-\mathrm{N}\left(\mathrm{CH}_{3}\right)_{2}\right), 2.62\left(\mathrm{t}, 3^{\prime}-\mathrm{H}\right), 2.64$ (brd, 2-H), 2.76 (dd, 2-H), 3.17 (t, 4'-H), 3.19 (s, 6$\left.\mathrm{CH}_{2} \mathrm{CH}\left(\mathrm{OCH}_{3}\right)_{2}\right), \quad 3.23$ (d, 2"-Heq), $3.26 \quad$ (s, 6$\left.\mathrm{CH}_{2} \mathrm{CH}\left(\mathrm{OCH}_{3}\right)_{2}\right), 3.46$ (dd, 4-H), $3.52\left(\mathrm{~s}, 4-\mathrm{OCH}_{3}\right), 3.82$ (br d, 5-H), 4.48 (dq, 5"-H), 4.58 (d, 4"-H), 4.66 (d, 1'-H), 4.81 (d, 1"-H), 4.92 (br d, 9-H), 4.96 (dd, 2'-H), 5.27 (br dd, 3-H), 5.39 (ddq, $\left.\mathrm{OCH}\left(\mathrm{CH}_{3}\right) \mathrm{CH}_{2} \mathrm{CHO}\right), 9.59$ (d, 10-H), 9.75 (t, $\left.\mathrm{OCH}\left(\mathrm{CH}_{3}\right) \mathrm{CH}_{2} \mathrm{CHO}\right)$.

(-)-(3R,4S,5S,6R,8R,9R,14R)-9-Acetoxy-5-[2-O-acetyl-4$O$-(3-O-acetyl-2,6-dideoxy-3-C-methyl-4- $O$-propionyl- $\alpha$-Lribo-hexopyranosyl)-3,6-dideoxy-3-dimethylamino- $\beta$-Dglucopyranosyloxy]-11-benzyl-6-(2,2-dimethoxyethyl)-4methoxy-8-methyl-3-propionyloxy-11-aza-pentadecan-14olide (16)

To a solution of $12(457 \mathrm{mg})$ in ethanol $(46 \mathrm{ml})$ were added acetic acid $(395 \mathrm{ml})$, benzylamine $(32 \mathrm{mg})$ and sodium cyanoborohydride $(75 \mathrm{mg})$ under ice cooling, and the mixture was stirred for 18 hours. Then sodium cyanoborohydride $(75 \mathrm{mg}$ ) was added, and the mixture was stirred at room temperature for 15 hours. The reaction mixture was diluted with ethyl acetate $(180 \mathrm{ml})$, washed successively with water $(50 \mathrm{ml})$, saturated aqueous sodium hydrogencarbonate solution $(50 \mathrm{ml})$ and saturated brine $(50 \mathrm{ml})$. The organic layer was dried over anhydrous sodium sulfate, and then filtered. The filtrate was concentrated under reduced pressure, and the resulting residue was purified by silica gel column chromatography (chloroform/ methanol $(60: 1 \sim 50: 1))$ to obtain $\mathbf{1 6}(98.3 \mathrm{mg}, 20 \%)$ as a colorless solid.

In the case of sequential reactions without purification of $\mathbf{1 2}$, two-step yield of $\mathbf{1 6}$ based on $\mathbf{1 3}$ was $10 \%$.

16: $[\alpha]_{\mathrm{D}}^{22}-79^{\circ}\left(c \quad 0.64, \mathrm{CHCl}_{3}\right)$; FAB-MS $m / z 1067$ $(\mathrm{M}+\mathrm{H})^{+}$as $\mathrm{C}_{54} \mathrm{H}_{86} \mathrm{~N}_{2} \mathrm{O}_{19} ;{ }^{1} \mathrm{H}-\mathrm{NMR}\left(300 \mathrm{MHz}, \mathrm{CDCl}_{3}\right) \delta$ : 0.90 (d, 18-H), 1.08 (d, 6"-H), $1.16\left(\mathrm{t}, 3-\mathrm{OCOCH}_{2} \mathrm{CH}_{3}\right.$ ), 1.19 (d, 15-H), 1.20 (t, 4"-OCOCH $\left.\mathrm{CH}_{3}\right), 1.25$ (d, 6'-H), $1.43\left(\mathrm{~s}, 3^{\prime \prime}-\mathrm{CH}_{3}\right), 1.59(\mathrm{~m}, 16-\mathrm{H}), 1.69$ (dd, 2"-Hax), 1.85 (m, 16-H), $2.02\left(\mathrm{~s}, 9-\mathrm{OCOCH}_{3}\right), 2.03\left(\mathrm{~s}, 3^{\prime \prime}-\mathrm{OCOCH}_{3}\right)$, $2.06\left(\mathrm{~s}, 2^{\prime}-\mathrm{OCOCH}_{3}\right), 2.45\left(\mathrm{~s}, 3^{\prime}-\mathrm{N}\left(\mathrm{CH}_{3}\right)_{2}\right), 2.84(\mathrm{dd}, 2-\mathrm{H})$, $3.16\left(\mathrm{~s}, 17-\mathrm{OCH}_{3}\right), 3.21$ (d, 2"-Heq), $3.27\left(\mathrm{~s}, 17-\mathrm{OCH}_{3}\right)$, 3.48 (br d, 4-H), 3.56 (s, 4- $\left.-\mathrm{OCH}_{3}\right), 3.59$ (d, $\left.\mathrm{C}_{6} \mathrm{H}_{5} \mathrm{CH}_{2}\right), 3.71$ (d, $\mathrm{C}_{6} \mathrm{H}_{5} \mathrm{CH}_{2}$ ), 3.91 (br d, 5-H), 4.52 (dq, 5"-H), 4.58 (d, 4"H), 4.69 (d, 1'-H), 4.82 (d, 1"-H), 4.90 (m, 9-H), 4.90 (m, 14-H), 4.99 (dd, 2'-H), 5.19 (br dd, 3-H), 7.29 (m, $\mathrm{C}_{6} \mathrm{H}_{5}$ ); ${ }^{13} \mathrm{C}-\mathrm{NMR}\left(100 \mathrm{MHz}, \mathrm{CDCl}_{3}\right) \delta$ : 17.0 (18-C), $17.3\left(6^{\prime \prime}-\mathrm{C}\right)$, 18.0 (6'-C), 20.2 (15-C), $22.1\left(3^{\prime \prime}-\mathrm{CH}_{3}\right), 30.4$ (7-C), 31.7 (16-C), 32.0 (13-C), 33.6 (8-C), 34.0 (6-C), 36.2 (2"-C), $36.8(2-\mathrm{C}), 49.2$ (12-C), $53.8(10-\mathrm{C}), 58.6\left(11-\mathrm{CH}_{2}\right), 61.3$ $\left(4-\mathrm{OCH}_{3}\right), 63.0\left(5^{\prime \prime}-\mathrm{C}\right), 68.0\left(3^{\prime}-\mathrm{C}\right), 69.9$ (3-C), 70.4 (14C), 70.8 (2'-C), 72.7 (5'-C), 75.1 (9-C), 75.8 (5-C), 77.7 (4"-C), 78.1 (3"-C), 79.2 (4'-C), 97.8 (1"-C), 100.6 (1'-C),
101.5 (17-C), 169.4 (1-C).

(-)-(3R,4S,5S,6R,8R,9R,14R)-9-Acetoxy-5-[4-O-(3-O-acetyl2,6-dideoxy-3-C-methyl-4- $O$-propionyl- $\alpha$-L-ribo-hexopyranosyl)-3,6-dideoxy-3-dimethylamino- $\beta$-D-glucopyranosyloxy]-11-benzyl-6-(2,2-dimethoxyethyl)-4-methoxy-8methyl-3-propionyloxy-11-aza-pentadecan-14-olide (17)

$16(20.0 \mathrm{mg})$ was dissolved in methanol and water $(9: 1)$ $(2.0 \mathrm{ml})$, and the mixture was stirred at $55^{\circ} \mathrm{C}$ for 24 hours. The reaction mixture was concentrated under reduced pressure, and the resulting residue was purified by preparative TLC (chloroform/methanol $(20: 1)$ ) to obtain $17(17.3 \mathrm{mg}, 90 \%)$ as a colorless solid.

17: $[\alpha]_{\mathrm{D}}^{21}-60^{\circ}\left(c \quad 0.61, \mathrm{CHCl}_{3}\right)$; FAB-MS $m / z 1025$ $(\mathrm{M}+\mathrm{H})^{+}$as $\mathrm{C}_{52} \mathrm{H}_{84} \mathrm{~N}_{2} \mathrm{O}_{18} ;{ }^{1} \mathrm{H}-\mathrm{NMR}\left(300 \mathrm{MHz}, \mathrm{CDCl}_{3}\right) \delta$ : 0.90 (d, 18-H), 1.10 (d, 6"-H), 1.15 (t, 3-OCOCH${ }_{2} \mathrm{CH}_{3}$ ), $1.16(\mathrm{~d}, 15-\mathrm{H}), 1.20\left(\mathrm{t}, 4^{\prime \prime}-\mathrm{OCOCH}_{2} \mathrm{CH}_{3}\right), 1.24\left(\mathrm{~d}, 6^{\prime}-\mathrm{H}\right)$, $1.43\left(\mathrm{~s}, 3^{\prime \prime}-\mathrm{CH}_{3}\right), 1.56(\mathrm{~m}, 16-\mathrm{H}), 1.69$ (dd, 2"-Hax), 2.03 (s, 9- $\left.-\mathrm{OCOCH}_{3}\right), 2.03\left(\mathrm{~s}, 3^{\prime \prime}-\mathrm{OCOCH}_{3}\right), 2.56\left(\mathrm{~s}, 3^{\prime}-\mathrm{N}\left(\mathrm{CH}_{3}\right)_{2}\right)$, $2.87(\mathrm{dd}, 2-\mathrm{H}), 3.17\left(\mathrm{~s}, 17-\mathrm{OCH}_{3}\right), 3.27\left(\mathrm{~s}, 17-\mathrm{OCH}_{3}\right), 3.44$ (dd, 2'-H), 3.54 (d, $\mathrm{C}_{6} \mathrm{H}_{5} \mathrm{CH}_{2}$ ), 3.57 (br d, 4-H), 3.62 (s, 4$\left.\mathrm{OCH}_{3}\right), 3.72\left(\mathrm{~d}, \mathrm{C}_{6} \mathrm{H}_{5} \mathrm{CH}_{2}\right), 3.92$ (br d, 5-H), 4.51 (d, 1'-H), 4.54 (dq, 5"-H), 4.60 (d, 4"-H), 4.87 (d, 1"-H), 4.87 (m, 14H), $4.91(\mathrm{~m}, 9-\mathrm{H}), 5.24$ (brdd, 3-H), $7.24\left(\mathrm{~m}, \mathrm{C}_{6} \mathrm{H}_{5}\right), 7.29$ (m, $\mathrm{C}_{6} \mathrm{H}_{5}$ ).

(-)-(3R,4S,5S,6R,8R,9R,14R)-9-Acetoxy-5-[4- $O-(3-O-$ acetyl-2,6-dideoxy-3- $C$-methyl-4- $O$-propionyl- $\alpha$-L-ribohexopyranosyl)-3,6-dideoxy-3-dimethylamino- $\beta$-D-glucopyranosyloxy]-11-benzyl-6-formylmethyl-4-methoxy-8methyl-3-propionyloxy-11-aza-pentadecan-14-olide (11)

To a solution of $\mathbf{1 7}(25 \mathrm{mg})$ in acetonitrile and water $(1: 1)$ $(0.75 \mathrm{ml})$ was added difluoroacetic acid $(35 \mu \mathrm{l})$, and the mixture was stirred at room temperature for 25 hours. The reaction mixture was diluted with chloroform $(40 \mathrm{ml})$, and washed with saturated aqueous sodium hydrogencarbonate solution $(25 \mathrm{ml})$. Further, the organic layer was successively washed with saturated aqueous sodium hydrogencarbonate solution $(35 \mathrm{ml})$ and saturated brine $(35 \mathrm{ml})$, dried over anhydrous sodium sulfate, and then filtered. The filtrate was concentrated under reduced pressure, and the resulting residue was purified by preparative TLC (chloroform/ methanol/aqueous ammonia $(15: 1: 0.1))$ to obtain 11 (20 mg, 84\%).

11: $[\alpha]_{\mathrm{D}}^{21}-70^{\circ}\left(c \quad 0.47, \mathrm{CHCl}_{3}\right)$; FAB-MS $m / z 979$ $(\mathrm{M}+\mathrm{H})^{+}$as $\mathrm{C}_{50} \mathrm{H}_{78} \mathrm{~N}_{2} \mathrm{O}_{17} ;{ }^{1} \mathrm{H}-\mathrm{NMR}\left(300 \mathrm{MHz}, \mathrm{CDCl}_{3}\right) \delta$ : 0.90 (d, 18-H), 1.10 (d, 6"-H), 1.16 (d, 6'-H), 1.17 (t, 3$\left.\mathrm{OCOCH}_{2} \mathrm{CH}_{3}\right), 1.19$ (d, 15-H), 1.20 (t, 4"-OCOCH $\mathrm{CH}_{3}$ ), 1.43 (s, 3"- $\mathrm{CH}_{3}$ ), 1.48 (br dd, 7-H), 1.70 (dd, 2"-Hax), 2.02 (s, 9- $\mathrm{OCOCH}_{3}$ ), 2.03 (s, 3"--OCOCH$\left.{ }_{3}\right), 2.15(\mathrm{~m}, 6-\mathrm{H}), 2.58$ (s, 3'-N( $\left.\left(\mathrm{CH}_{3}\right)_{2}\right), 2.85$ (dd, 2-H), 2.95 (dd, 16-H), 3.24 (d, 2"- 
Heq), 3.25 (m, 4'-H), 3.25 (m, 5'-H), 3.38 (dd, 2'-H), 3.55 (d, $\mathrm{C}_{6} \mathrm{H}_{5} \mathrm{CH}_{2}$ ), 3.61 (s, 4-OCH $\left.{ }_{3}\right), 3.64$ (br d, 4-H), 3.73 (d, $\mathrm{C}_{6} \mathrm{H}_{5} \mathrm{CH}_{2}$ ), 3.94 (br d, 5-H), 4.48 (d, 1'-H), 4.48 (dq, 5"-H), 4.60 (d, 4"-H), 4.78 (br q, 14-H), 4.89 (d, 1"-H), 4.89 (m, 9$\mathrm{H}), 5.38$ (br dd, 3-H), $7.25\left(\mathrm{~m}, \mathrm{C}_{6} \mathrm{H}_{5}\right), 7.29\left(\mathrm{~m}, \mathrm{C}_{6} \mathrm{H}_{5}\right), 9.65$ (s, 17-H).

$(-)-(3 R, 4 S, 5 S, 6 R, 8 R, 9 R, 15 R)-9$-Acetoxy-5-[2-O-acetyl-4$O$-(3-O-acetyl-2,6-dideoxy-3- $C$-methyl-4- $O$-propionyl- $\alpha$-Lribo-hexopyranosyl)-3,6-dideoxy-3-dimethylamino- $\beta$-Dglucopyranosyloxy]-6-(2,2-dimethoxyethyl)-4-methoxy8,11,12-trimethyl-3-propionyloxy-11,12-diaza-hexadecan15-olide (18)

Reaction of 12 with 1,2-dimethylhydrazine dihydrochloride gave $\mathbf{1 8}$ in $13 \%$ yield by a similar procedure to $\mathbf{1 6}$.

18: $[\alpha]_{\mathrm{D}}^{24}-71^{\circ}\left(c 0.76, \mathrm{CHCl}_{3}\right)$; FAB-MS $m / z 1019(\mathrm{M})^{+}$ as $\mathrm{C}_{49} \mathrm{H}_{85} \mathrm{~N}_{3} \mathrm{O}_{19} ;{ }^{1} \mathrm{H}-\mathrm{NMR}\left(300 \mathrm{MHz}, \mathrm{CDCl}_{3}\right) \delta: 0.93(\mathrm{~d}$, 19-H), 1.07 (d, 6"-H), 1.15 (t, 3-OCOCH${ }_{2} \mathrm{CH}_{3}$ ), 1.19 (t, 4"$\left.\mathrm{OCOCH}_{2} \mathrm{CH}_{3}\right), 1.20\left(\mathrm{~d}, 6^{\prime}-\mathrm{H}\right), 1.26(\mathrm{~d}, 16-\mathrm{H}), 1.41\left(\mathrm{~s}, 3^{\prime \prime}-\right.$ $\left.\mathrm{CH}_{3}\right), 1.52$ (m, 17-H), 1.67 (dd, 2"-Hax), $1.71(\mathrm{~m}, 8-\mathrm{H})$, $2.03\left(\mathrm{~s}, 9-\mathrm{OCOCH}_{3}\right), 2.03\left(\mathrm{~s}, 3^{\prime \prime}-\mathrm{OCOCH}_{3}\right), 2.05\left(\mathrm{~s}, 2^{\prime}-\right.$ $\left.\mathrm{OCOCH}_{3}\right), 2.22\left(\mathrm{~s}, 11-\mathrm{NCH}_{3}\right), 2.22\left(\mathrm{~s}, 12-\mathrm{NCH}_{3}\right), 2.43(\mathrm{~s}$, $\left.3^{\prime}-\mathrm{N}\left(\mathrm{CH}_{3}\right)_{2}\right), 2.61\left(\mathrm{t}, 3^{\prime}-\mathrm{H}\right), 2.75(\mathrm{dd}, 2-\mathrm{H}), 3.14\left(\mathrm{t}, 4^{\prime}-\mathrm{H}\right)$, 3.19 (d, 2"-Heq), 3.20 (s, 18- $\left.\mathrm{OCH}_{3}\right), 3.25$ (s, 18- $\left.\mathrm{OCH}_{3}\right)$, 3.43 (br d, 4-H), 3.57 (s, 4-- $\mathrm{OCH}_{3}$ ), 3.85 (br d, 5-H), 4.45 (t, 18-H), 4.48 (dq, 5"-H), 4.57 (d, 4"'-H), 4.67 (d, 1'-H), 4.81 (d, 1"-H), 4.90 (m, 15-H), 4.98 (dd, 2'-H), 5.05 (br dd, 9-H), $5.22(\mathrm{~m}, 3-\mathrm{H})$.

(-)- $(3 R, 4 S, 5 S, 6 R, 8 R, 9 R, 15 R)-9$-Acetoxy-5-[4-O-(3-Oacetyl-2,6-dideoxy-3-C-methyl-4- $O$-propionyl- $\alpha$-L-ribohexopyranosyl)-3,6-dideoxy-3-dimethylamino- $\beta$-Dglucopyranosyloxy]-6-(2,2-dimethoxyethyl)-4-methoxy8,11,12-trimethyl-3-propionyloxy-11,12-diaza-hexadecan15-olide (19)

Reaction of 18 with aqueous methanol gave 19 in $79 \%$ yield by a similar procedure to 17 .

19: $[\alpha]_{\mathrm{D}}^{22}-52^{\circ}\left(c \quad 0.77, \mathrm{CHCl}_{3}\right)$; FAB-MS $m / z 978$ $(\mathrm{M}+\mathrm{H})^{+}$as $\mathrm{C}_{47} \mathrm{H}_{83} \mathrm{~N}_{3} \mathrm{O}_{18} ;{ }^{1} \mathrm{H}-\mathrm{NMR}\left(300 \mathrm{MHz}, \mathrm{CDCl}_{3}\right) \delta$ : 0.95 (d, 19-H), 1.09 (d, 6"-H), 1.15 (t, 3-OCOCH $\mathrm{CH}_{3}$ ), 1.19 (t, 4"-OCOCH $\left.\mathrm{CH}_{3}\right), 1.21\left(\mathrm{~d}, 6^{\prime}-\mathrm{H}\right), 1.26(\mathrm{~d}, 16-\mathrm{H})$, 1.42 (s, 3"-- $\left.\mathrm{CH}_{3}\right), 1.59$ (m, 17-H), 1.70 (dd, 2"-Hax), 2.02 (s, 9- $\left.\mathrm{OCOCH}_{3}\right), 2.05\left(\mathrm{~s}, 3^{\prime \prime}-\mathrm{OCOCH}_{3}\right), 2.37,2.38$ (each q, 4"$\left.\mathrm{OCOCH}_{2} \mathrm{CH}_{3}\right), 2.42\left(\mathrm{~s}, 11-\mathrm{NCH}_{3}\right), 2.42\left(\mathrm{~s}, 12-\mathrm{NCH}_{3}\right), 2.43$ (br q, 3-OCOCH$\left.{ }_{2} \mathrm{CH}_{3}\right), 2.54$ (s, 3'-N( $\left.\left(\mathrm{CH}_{3}\right)_{2}\right), 2.78$ (dd, 2$\mathrm{H}), 3.22$ (s, 18- $\left.\mathrm{OCH}_{3}\right), 3.24$ (d, 2"-Heq), 3.27 (s, 18- $\mathrm{OCH}_{3}$ ), $3.46\left(\mathrm{dd}, 2^{\prime}-\mathrm{H}\right), 3.50(\mathrm{brd}, 4-\mathrm{H}), 3.63\left(\mathrm{~s}, 4-\mathrm{OCH}_{3}\right), 3.88$ (br d, 5-H), 4.45 (t, 18-H), $4.46\left(\mathrm{~d}, 1^{\prime}-\mathrm{H}\right), 4.56\left(\mathrm{~m}, 5^{\prime \prime}-\mathrm{H}\right)$, $4.59\left(\mathrm{~d}, 4^{\prime \prime}-\mathrm{H}\right), 4.85\left(\mathrm{~d}, \mathrm{l}^{\prime \prime}-\mathrm{H}\right), 4.94(\mathrm{~m}, 15-\mathrm{H}), 5.05$ (m, 9H), $5.31(\mathrm{~m}, 3-\mathrm{H})$.
(-)-(3R,4S,5S,6R,8R,9R,15R)-9-Acetoxy-5-[4-O-(3-Oacetyl-2,6-dideoxy-3- $C$-methyl-4- $O$-propionyl- $\alpha$-L-ribohexopyranosyl)-3,6-dideoxy-3-dimethylamino- $\beta$-D-glucopyranosyloxy]-6-formylmethyl-4-methoxy-8,11,12-trimethyl3-propionyloxy-11,12-diaza-hexadecan-15-olide (20)

Reaction of $\mathbf{1 9}$ with aqueous difluoroacetic acid gave $\mathbf{2 0}$ in $94 \%$ yield by a similar procedure to 11 .

20: $[\alpha]_{\mathrm{D}}^{22}-74^{\circ}\left(c \quad 0.42, \mathrm{CHCl}_{3}\right)$; FAB-MS $\mathrm{m} / \mathrm{z} 932$ $(\mathrm{M}+\mathrm{H})^{+}$as $\mathrm{C}_{45} \mathrm{H}_{77} \mathrm{~N}_{3} \mathrm{O}_{17} ;{ }^{1} \mathrm{H}-\mathrm{NMR}\left(300 \mathrm{MHz}, \mathrm{CDCl}_{3}\right) \delta$ : 0.97 (d, 19-H), 1.09 (d, 6"-H), 1.15 (d, 6'-H), 1.17 (t, 3$\mathrm{OCOCH}_{2} \mathrm{CH}_{3}$ ), 1.19 (t, 4"-OCOCH${ }_{2} \mathrm{CH}_{3}$ ), 1.26 (d, 16-H), $1.42\left(\mathrm{~s}, 3^{\prime \prime}-\mathrm{CH}_{3}\right), 1.70$ (dd, 2"-Hax), 1.76 (m, 8-H), 2.02 (s, 9- $\left.\mathrm{OCOCH}_{3}\right), 2.05\left(\mathrm{~s}, 3^{\prime \prime}-\mathrm{OCOCH}_{3}\right), 2.22\left(\mathrm{~s}, 11-\mathrm{NCH}_{3}\right)$, $2.25\left(\mathrm{~s}, 12-\mathrm{NCH}_{3}\right), 2.56\left(\mathrm{~s}, 3^{\prime}-\mathrm{N}\left(\mathrm{CH}_{3}\right)_{2}\right), 3.04(\mathrm{dd}, 17-\mathrm{H})$, 3.23 (m, 4'-H), 3.23 (m, 5'-H), 3.23 (d, 2"'-Heq), 3.40 (dd, $\left.2^{\prime}-\mathrm{H}\right), 3.58\left(\mathrm{~s}, 4-\mathrm{OCH}_{3}\right), 3.99$ (br d, 5-H), 4.42 (d, 1'-H), 4.50 (dq, 5"-H), 4.59 (d, 4"-H), 4.86 (d, 1"'-H), 4.90 (m, 15H), 5.00 (m, 9-H), 5.38 (br dd, 3-H), 9.64 (s, 18-H).

(-)-(3R,4S,5S,6R,8R,9R,14R)-9-Acetoxy-5-[2-O-acetyl-4$O$-(3-O-acetyl-2,6-dideoxy-3- $C$-methyl-4- $O$-propionyl- $\alpha$-Lribo-hexopyranosyl)-3,6-dideoxy-3-dimethylamino- $\beta$-Dglucopyranosyloxy]-6-(2,2-dimethoxyethyl)-4-methoxy-11(4-methoxybenzyl)-8-methyl-3-propionyloxy-11-azapentadecan-14-olide (21)

Reaction of 12 with 4-methoxybenzylamine gave $\mathbf{2 1}$ in $8.8 \%$ yield by a similar procedure to $\mathbf{1 6}$.

21: $[\alpha]_{\mathrm{D}}^{25}-87^{\circ}$ (c 1.0, $\mathrm{CHCl}_{3}$ ); FAB-MS $\mathrm{m} / \mathrm{z} 1097$ $(\mathrm{M}+\mathrm{H})^{+}$as $\mathrm{C}_{55} \mathrm{H}_{88} \mathrm{~N}_{2} \mathrm{O}_{20} ;{ }^{1} \mathrm{H}-\mathrm{NMR}\left(300 \mathrm{MHz}, \mathrm{CDCl}_{3}\right) \delta$ : 0.89 (d, 18-H), 1.07 (d, 6"-H), 1.15 (t, 3-OCOCH${ }_{2} \mathrm{CH}_{3}$ ), $1.17(\mathrm{~d}, 15-\mathrm{H}), 1.19\left(\mathrm{t}, 4^{\prime \prime}-\mathrm{OCOCH}_{2} \mathrm{CH}_{3}\right), 1.21\left(\mathrm{~d}, 6^{\prime}-\mathrm{H}\right)$, $1.42\left(\mathrm{~s}, 3^{\prime \prime}-\mathrm{CH}_{3}\right), 1.54$ (br dd, 16-H), 1.68 (dd, 2"-Hax), 2.02

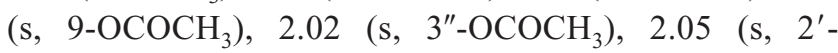
$\left.\mathrm{OCOCH}_{3}\right), 2.44\left(\mathrm{~s}, 3^{\prime}-\mathrm{N}\left(\mathrm{CH}_{3}\right)_{2}\right), 2.83(\mathrm{dd}, 2-\mathrm{H}), 3.14(\mathrm{~s}$, 17- $\mathrm{OCH}_{3}$ ), 3.15 (m, 4'-H), 3.15 (m, 5'-H), 3.19 (d, 2"-Heq), $3.26\left(\mathrm{~s}, 17-\mathrm{OCH}_{3}\right), 3.48$ (br d, 4-H), $3.50\left(\mathrm{~d}, \mathrm{C}_{6} \mathrm{H}_{4} \mathrm{CH}_{2}\right.$ ), $3.55\left(\mathrm{~s}, 4-\mathrm{OCH}_{3}\right), 3.64\left(\mathrm{~d}, \mathrm{C}_{6} \mathrm{H}_{4} \mathrm{CH}_{2}\right), 3.79\left(\mathrm{~s}, \mathrm{CH}_{3} \mathrm{OC}_{6} \mathrm{H}_{4}\right)$, 3.90 (br d, 5-H), 4.49 (dq, 5"-H), 4.57 (d, 4"-H), 4.68 (d, 1'H), 4.81 (d, 1"-H), 4.89 (m, 9-H), 4.89 (m, 14-H), 4.98 (dd, $\left.2^{\prime}-\mathrm{H}\right), 5.19(\mathrm{~m}, 3-\mathrm{H}), 6.83\left(\mathrm{~d}, \mathrm{C}_{6} \mathrm{H}_{4}\right), 7.19\left(\mathrm{~d}, \mathrm{C}_{6} \mathrm{H}_{4}\right)$.

(-)-(3R,4S,5S,6R,8R,9R,14R)-5-[4-O-(3-O-Acetyl-2,6dideoxy-3-C-methyl-4- $O$-propionyl- $\alpha$-L-ribo-hexopyranosyl)-3,6-dideoxy-3-dimethylamino- $\beta$-D-glucopyranosyloxy]-6-(2,2-dimethoxyethyl)-9-hydroxy-4-methoxy-8methyl-3-propionyloxy-11-aza-pentadecan-14-olide (22)

Reaction of $\mathbf{2 1}$ with aqueous methanol gave $N$-(4methoxybenzyl) derivative of $\mathbf{2 2}$ in $52 \%$ yield by a similar procedure to 17.

$N$-(4-Methoxybenzyl) derivative of 22: $[\alpha]_{\mathrm{D}}^{26}-68^{\circ}(c$ $\left.0.60, \mathrm{CHCl}_{3}\right)$; FAB-MS $m / z 1013(\mathrm{M}+\mathrm{H})^{+}$as $\mathrm{C}_{51} \mathrm{H}_{84} \mathrm{~N}_{2} \mathrm{O}_{18}$; 
${ }^{1} \mathrm{H}-\mathrm{NMR}\left(300 \mathrm{MHz}, \mathrm{CDCl}_{3}\right) \delta: 0.93(\mathrm{~d}, 18-\mathrm{H}), 1.10$ (d, 6"$\mathrm{H}), 1.14$ (t, 3-OCOCH $\left.\mathrm{CH}_{3}\right), 1.20$ (t, 4"-OCOCH $\mathrm{CH}_{3}$ ), $1.21\left(\mathrm{~d}, 6^{\prime}-\mathrm{H}\right), 1.22(\mathrm{~d}, 15-\mathrm{H}), 1.43\left(\mathrm{~s}, 3^{\prime \prime}-\mathrm{CH}_{3}\right), 1.61$ (br dd, 16-H), 1.70 (dd, 2"-Hax), 2.02 (s, 3"-OCOCH 3 ), 2.56 (s, 3'$\left.\mathrm{N}\left(\mathrm{CH}_{3}\right)_{2}\right), 2.60$ (dd, 2-H), 2.84 (dd, 2-H), 3.13 (s, 17$\mathrm{OCH}_{3}$ ), 3.22 (m, 4'-H), 3.22 (m, 5'-H), 3.23 (d, 2"-Heq), $3.27\left(\mathrm{~s}, 17-\mathrm{OCH}_{3}\right), 3.41\left(\mathrm{dd}, 2^{\prime}-\mathrm{H}\right), 3.43\left(\mathrm{~d}, \mathrm{C}_{6} \mathrm{H}_{4} \mathrm{CH}_{2}\right), 3.61$ (s, 4- $\left.\mathrm{OCH}_{3}\right), 3.63(\mathrm{dd}, 4-\mathrm{H}), 3.73\left(\mathrm{~d}, \mathrm{C}_{6} \mathrm{H}_{4} \mathrm{CH}_{2}\right), 3.81$ (s, $\left.\mathrm{C}_{6} \mathrm{H}_{4} \mathrm{OMe}\right), 3.90$ (br d, 5-H), 4.49 (d, 1'-H), 4.50 (t, 17-H), 4.55 (dq, 5"-H), 4.59 (d, 4"-H), $4.82(\mathrm{~m}, 14-\mathrm{H}), 4.86$ (d, 1"$\mathrm{H}), 5.24$ (br dd, 3-H), $6.86\left(\mathrm{~d}, \mathrm{C}_{6} \mathrm{H}_{4}\right), 7.20\left(\mathrm{~d}, \mathrm{C}_{6} \mathrm{H}_{4}\right)$.

To a solution of $\mathrm{N}$-(4-methoxybenzyl) derivative of $\mathbf{2 2}$ $(63.0 \mathrm{mg})$ in 1,4-dioxane $(1.5 \mathrm{ml})$ was added $10 \%$ Pd-C catalyst $(6.3 \mathrm{mg})$ suspended in ethanol $(1.0 \mathrm{ml})$. The atmosphere in the reaction vessel was replaced with hydrogen, and the mixture was stirred at room temperature for 135 minutes. $10 \%$ Pd-C catalyst $(12.6 \mathrm{mg})$ suspended in ethanol $(0.5 \mathrm{ml})$ was added, and the reaction mixture was stirred for 165 minutes. Then, $10 \%$ Pd-C catalyst $(6.3 \mathrm{mg})$ suspended in ethanol $(0.5 \mathrm{ml})$ was added, and the mixture was stirred for 1 hour, and the catalyst was removed by filtration. The filtrate was concentrated under reduced pressure, and the resulting residue was purified by preparative TLC (chloroform/methanol/aqueous ammonia (10:1:0.1)) to obtain 22 (32.0 mg, 58\%).

Two-step yield of $\mathbf{2 2}$ based on $\mathbf{2 1}$ was $30 \%$.

22: $[\alpha]_{\mathrm{D}}^{25}-67^{\circ}\left(c \quad 0.80, \mathrm{CHCl}_{3}\right.$ ); FAB-MS $\mathrm{m} / \mathrm{z} 893$ $(\mathrm{M}+\mathrm{H})^{+}$as $\mathrm{C}_{43} \mathrm{H}_{76} \mathrm{~N}_{2} \mathrm{O}_{17} ;{ }^{1} \mathrm{H}-\mathrm{NMR}\left(300 \mathrm{MHz}, \mathrm{CDCl}_{3}\right) \delta$ : $0.91(\mathrm{~d}, 18-\mathrm{H}), 1.08\left(\mathrm{~d}, 6\right.$ "'-H), 1.14 (t, 3- $\left.\mathrm{OCOCH}_{2} \mathrm{CH}_{3}\right)$, 1.19 (t, 4"-OCOCH$\left.{ }_{2} \mathrm{CH}_{3}\right), 1.21$ (d, 6'-H), 1.27 (d, 15-H), $1.41\left(\mathrm{~s}, 3^{\prime \prime}-\mathrm{CH}_{3}\right), 1.69$ (dd, 2"-Hax), 2.01 (s, 3"-OCOCH${ }_{3}$ ), $2.54\left(\mathrm{~s}, 3^{\prime}-\mathrm{N}\left(\mathrm{CH}_{3}\right)_{2}\right), 2.60$ (dd, 2-H), 2.72 (dd, 2-H), 2.80 (br d, 10-H), 2.91 (m, 12-H), 3.15 (s, 17- $\left.\mathrm{OCH}_{3}\right), 3.22$ (d, 2"Heq), 3.25 (s, 17- $\left.-\mathrm{OCH}_{3}\right), 3.41$ (dd, 2'-H), $3.62\left(\mathrm{~s}, 4-\mathrm{OCH}_{3}\right)$, 3.86 (br d, 5-H), 4.46 (brt, 17-H), 4.46 (d, 1'-H), 4.52 (dq, 5"-H), 4.58 (d, 4"-H), 4.85 (d, 1"-H), 4.96 (m, 14-H), 5.17 (br dd, 3-H).

(-)-(3R,4S,5S,6R,8R,9R,14R)-5-[4-O-(3-O-Acetyl-2,6dideoxy-3-C-methyl-4- $O$-propionyl- $\alpha$-L-ribo-hexopyranosyl)-3,6-dideoxy-3-dimethylamino- $\beta$-D-glucopyranosyloxy]-9-O,11-carbonyl-6-(2,2-dimethoxyethyl)-4-methoxy8-methyl-3-propionyloxy-11-aza-pentadecan-14-olide (23)

To a solution of $22(30.0 \mathrm{mg})$ in dichloromethane $(1.0 \mathrm{ml})$ were added triethylamine $(42 \mu \mathrm{l})$ and a solution of triphosgene $(11 \mathrm{mg})$ in dichloromethane $(0.50 \mathrm{ml})$ under ice cooling, and the mixture was stirred for 1.5 hours at the same temperature. Chloroform $(20 \mathrm{ml})$ and saturated aqueous sodium hydrogencarbonate solution $(15 \mathrm{ml})$ were added to the resulting mixture, and the organic layer was separated. The organic layer was washed with saturated brine $(15 \mathrm{ml})$, and dried over anhydrous sodium sulfate, and then filtered. The filtrate was concentrated under reduced pressure, and the resulting residue was purified by preparative TLC (ethyl acetate/methanol/aqueous ammonia ( $35: 1: 0.1)$ ) to obtain 23 (23.0 $\mathrm{mg}, 74 \%)$.

23: $[\alpha]_{\mathrm{D}}^{26}-48^{\circ}\left(c \quad 0.61, \mathrm{CHCl}_{3}\right)$; FAB-MS $m / z 919$ $(\mathrm{M}+\mathrm{H})^{+}$as $\mathrm{C}_{44} \mathrm{H}_{74} \mathrm{~N}_{2} \mathrm{O}_{18} ;{ }^{1} \mathrm{H}-\mathrm{NMR}\left(300 \mathrm{MHz}, \mathrm{CDCl}_{3}\right) \delta$ : 1.02 (d, 18-H), 1.08 (d, 6"-H), 1.13 (t, 3- $-\mathrm{OCOCH}_{2} \mathrm{CH}_{3}$ ), 1.19 (t, 4"-OCOCH$\left.{ }_{2} \mathrm{CH}_{3}\right), 1.20$ (d, 6'-H), 1.32 (d, 15-H), 1.41 (s, 3"- $\left.-\mathrm{CH}_{3}\right), 1.48$ (m, 16-H), 1.68 (dd, 2"-Hax), 1.82 (m, 13-H), $2.01\left(\mathrm{~s}, 3^{\prime \prime}-\mathrm{OCOCH}_{3}\right), 2.08(\mathrm{~m}, 13-\mathrm{H}), 2.37$ (q, 4"-OCOCH${ }_{2} \mathrm{CH}_{3}$ ), 2.40 (t, 3'-H), 2.42 (q, 3-OCOCH${ }_{2} \mathrm{CH}_{3}$ ), $2.54\left(\mathrm{~s}, 3^{\prime}-\mathrm{N}\left(\mathrm{CH}_{3}\right)_{2}\right), 2.62$ (dd, 2-H), 2.79 (dd, 2-H), 3.04 (br dd, 12-H), 3.15 (t, 4'-H), 3.20 (d, 2"-Heq), 3.21 (s, 17$\left.\mathrm{OCH}_{3}\right), 3.25$ (s, 17- $\left.-\mathrm{OCH}_{3}\right), 3.39$ (dd, 4-H), 3.46 (dd, 10-H), 3.54 (dd, 2'-H), 3.56 (s, 4- $\left.-\mathrm{OCH}_{3}\right), 3.81$ (br d, 5-H), 3.83 (m, 12-H), 4.26 (br dd, 9-H), 4.46 (t, 17-H), 4.47 (d, 1'-H), 4.57 (m, 4"-H), 4.57 (m, 5"-H), 4.83 (d, 1"-H), 5.04 (br dq, 14H), 5.35 (br dd, 3-H).

(-)-(3R,4S,5S,6R,8R,9R,14R)-5-[4-O-(3-O-Acetyl-2,6dideoxy-3-C-methyl-4- $O$-propionyl- $\alpha$-L-ribo-hexopyranosyl)-3,6-dideoxy-3-dimethylamino- $\beta$-D-glucopyranosyloxy]-9-O,11-carbonyl-6-formylmethyl-4-methoxy-8-methyl3-propionyloxy-11-aza-pentadecan-14-olide (24)

Reaction of $\mathbf{2 3}$ with aqueous difluoroacetic acid gave $\mathbf{2 4}$ in $86 \%$ yield by a similar procedure to 11 .

24: $[\alpha]_{\mathrm{D}}^{26}-55^{\circ}(c) 0.51, \mathrm{CHCl}_{3}$ ); FAB-MS $m / z 873$ $(\mathrm{M}+\mathrm{H})^{+}$as $\mathrm{C}_{42} \mathrm{H}_{68} \mathrm{~N}_{2} \mathrm{O}_{17} ;{ }^{1} \mathrm{H}-\mathrm{NMR}\left(300 \mathrm{MHz}, \mathrm{CDCl}_{3}\right) \delta$ : $1.03(\mathrm{~d}, 18-\mathrm{H}), 1.08$ (d, 6"-H), $1.14\left(\mathrm{~d}, 6^{\prime}-\mathrm{H}\right), 1.17$ (t, 3$\mathrm{OCOCH}_{2} \mathrm{CH}_{3}$ ), 1.19 (t, 4"-OCOCH${ }_{2} \mathrm{CH}_{3}$ ), 1.33 (d, 15-H), $1.41\left(\mathrm{~s}, 3^{\prime \prime}-\mathrm{CH}_{3}\right), 1.63(\mathrm{~m}, 8-\mathrm{H}), 1.68$ (dd, 2"-Hax), 1.81 (m, 6-H), 1.85 (m, 13-H), 2.01 (s, 3"-OCOCH $\left.{ }_{3}\right), 2.09$ (m, 13H), 2.24 (dd, 16-H), 2.40 (q, 3-OCOCH${ }_{2} \mathrm{CH}_{3}$ ), 2.40 (t, 3'$\mathrm{H}), 2.45$ (q, 4"-OCOCH $\left.\mathrm{CH}_{3}\right), 2.55\left(\mathrm{~s}, 3^{\prime}-\mathrm{N}\left(\mathrm{CH}_{3}\right)_{2}\right), 2.67$ (dd, 2-H), 2.80 (dd, 2-H), 3.02 (m, 12-H), 3.09 (dd, 16-H), 3.14 (t, 4'-H), 3.21 (d, 2"'-Heq), 3.38 (dd, 4-H), 3.49 (dd, 2'H), $3.51(\mathrm{~m}, 10-\mathrm{H}), 3.57\left(\mathrm{~s}, 4-\mathrm{OCH}_{3}\right), 3.90(\mathrm{~m}, 12-\mathrm{H}), 3.92$ (br d, 5-H), 4.21 (br dd, 9-H), 4.44 (d, 1'-H), 4.55 (m, 5"-H), 4.57 (m, 4"-H), 4.83 (d, 1"-H), 5.03 (m, 14-H), 5.43 (br dd, 3-H), 9.64 (s, 17-H).

(-)-(3R,4S,5S,6R,8R,9R,14R)-9-Acetoxy-5-[2-O-acetyl-4$O$-(3-O-acetyl-2,6-dideoxy-3- $C$-methyl-4- $O$-propionyl- $\alpha$-Lribo-hexopyranosyl)-3,6-dideoxy-3-dimethylamino- $\beta$-Dglucopyranosyloxy]-6-(2,2-dimethoxyethyl)-4-methoxy8,11-dimethyl-3-propionyloxy-11-aza-pentadecan-14-olide (25)

Reaction of $\mathbf{1 2}$ with methylamine hydrochloride gave $\mathbf{2 5}$ in $11 \%$ yield by a similar procedure to 16 .

25: $\left.[\alpha]_{\mathrm{D}}^{22}-72^{\circ}(c) 1.0, \mathrm{CHCl}_{3}\right)$; FAB-MS $m / z 991$ 
$(\mathrm{M}+\mathrm{H})^{+}$as $\mathrm{C}_{48} \mathrm{H}_{82} \mathrm{~N}_{2} \mathrm{O}_{19} ;{ }^{1} \mathrm{H}-\mathrm{NMR}\left(300 \mathrm{MHz}, \mathrm{CDCl}_{3}\right) \delta$ : 0.94 (d, 18-H), 1.07 (d, 6"-H), 1.14 (t, 3- $-\mathrm{OCOCH}_{2} \mathrm{CH}_{3}$ ), 1.19 (t, 4"-OCOCH${ }_{2} \mathrm{CH}_{3}$ ), 1.20 (d, 6'-H), 1.24 (d, 15-H), 1.41 (s, 3"-- $\mathrm{CH}_{3}$ ), 1.53 (br dd, 16-H), 1.67 (dd, 2"-Hax), 1.82 (br s, 16-H), $2.02\left(\mathrm{~s}, 9-\mathrm{OCOCH}_{3}\right), 2.03\left(\mathrm{~s}, 3^{\prime \prime}-\mathrm{OCOCH}_{3}\right)$, $2.04\left(\mathrm{~s}, 2^{\prime}-\mathrm{OCOCH}_{3}\right), 2.26\left(\mathrm{~s}, 11-\mathrm{NCH}_{3}\right), 2.43\left(\mathrm{~s}, 3^{\prime}-\right.$ $\left.\mathrm{N}\left(\mathrm{CH}_{3}\right)_{2}\right), 2.58(\mathrm{dd}, 2-\mathrm{H}), 2.62\left(\mathrm{t}, 3^{\prime}-\mathrm{H}\right), 2.82(\mathrm{dd}, 2-\mathrm{H})$, 3.14 (t, 4'-H), 3.17 (s, 17- $\left.-\mathrm{OCH}_{3}\right), 3.19$ (d, 2"-Heq), 3.25 (s, $\left.17-\mathrm{OCH}_{3}\right), 3.35$ (br d, 4-H), 3.57 (s, 4- $\mathrm{OCH}_{3}$ ), 3.86 (br d, 5H), 4.48 (dq, 5"-H), 4.56 (d, 4"-H), 4.68 (d, 1'-H), 4.81 (d, 1"-H), 4.98 (dd, 2'-H), 5.12 (m, 14-H), 5.16 (m, 3-H).

(-)-(3R,4S,5S,6R,8R,9R,14R)-5-[4-O-(3-O-Acetyl-2,6dideoxy-3-C-methyl-4- $O$-propionyl- $\alpha$-L-ribo-hexopyranosyl)-3,6-dideoxy-3-dimethylamino- $\beta$-D-glucopyranosyloxy]-6-(2,2-dimethoxyethyl)-9-hydroxy-4-methoxy-8,11dimethyl-3-propionyloxy-11-aza-pentadecan-14-olide (26) $25(50.0 \mathrm{mg})$ was dissolved in methanol $(2.0 \mathrm{ml})$, and the mixture was stirred at room temperature for 72 hours. The reaction mixture was concentrated under reduced pressure, and the resulting residue was purified by preparative TLC (chloroform/methanol/aqueous ammonia $(10: 1: 0.1)$ ) to obtain 26 (27.2 mg, 59\%).

26: $[\alpha]_{\mathrm{D}}^{23}-60^{\circ}\left(c \quad 0.4, \mathrm{CHCl}_{3}\right)$; FAB-MS $m / z 907$ $(\mathrm{M}+\mathrm{H})^{+}$as $\mathrm{C}_{44} \mathrm{H}_{78} \mathrm{~N}_{2} \mathrm{O}_{17} ;{ }^{1} \mathrm{H}-\mathrm{NMR}\left(300 \mathrm{MHz}, \mathrm{CDCl}_{3}\right) \delta$ : 0.97 (d, 18-H), 1.09 (d, 6"-H), 1.13 (t, 3-OCOCH${ }_{2} \mathrm{CH}_{3}$ ), 1.20 (t, 4"-OCOCH $\left.\mathrm{CH}_{3}\right), 1.21\left(\mathrm{~d}, 6^{\prime}-\mathrm{H}\right), 1.27(\mathrm{~d}, 15-\mathrm{H})$, $1.42\left(\mathrm{~s}, 3^{\prime \prime}-\mathrm{CH}_{3}\right), 1.53(\mathrm{~m}, 8-\mathrm{H}), 1.60(\mathrm{~m}, 16-\mathrm{H}), 1.69$ (dd, 2"-Hax), 1.90 (br dd, 16-H), 2.02 (s, 3"-OCOCH $), 2.39$ (s, $\left.11-\mathrm{NCH}_{3}\right), 2.55\left(\mathrm{~s}, 3^{\prime}-\mathrm{N}\left(\mathrm{CH}_{3}\right)_{2}\right), 2.62(\mathrm{dd}, 2-\mathrm{H}), 2.79$ (dd, 2-H), 3.16 (s, 17- $\mathrm{OCH}_{3}$ ), 3.23 (d, 2"-Heq), 3.27 (s, 17$\mathrm{OCH}_{3}$ ), 3.43 (dd, 2'-H), 3.54 (br d, 4-H), 3.60 (s, 4- $\mathrm{OCH}_{3}$ ), 3.87 (br d, 5-H), 4.47 (d, 1'-H), 4.57 (d, 4"-H), 4.85 (d, 1"H), 4.90 (m, 14-H), 5.27 (br dd, 3-H).

(-)-(3R,4S,5S,6R,8R,9R,14R)-5-[4-O-(3-O-Acetyl-2,6dideoxy-3-C-methyl-4- $O$-propionyl- $\alpha$-L-ribo-hexopyranosyl)-3,6-dideoxy-3-dimethylamino- $\beta$-D-glucopyranosyloxy]-6-formylmethyl-9-hydroxy-4-methoxy-8,11-dimethyl-3-propionyloxy-11-aza-pentadecan-14-olide (27) Reaction of $\mathbf{2 6}$ with aqueous difluoroacetic acid gave $\mathbf{2 7}$ in $70 \%$ yield by a similar procedure to $\mathbf{1 1}$.

27: $[\alpha]_{\mathrm{D}}^{25}-72^{\circ}\left(c \quad 0.48, \mathrm{CHCl}_{3}\right.$ ); FAB-MS $m / z 861$ $(\mathrm{M}+\mathrm{H})^{+}$as $\mathrm{C}_{42} \mathrm{H}_{72} \mathrm{~N}_{2} \mathrm{O}_{16} ;{ }^{1} \mathrm{H}-\mathrm{NMR}\left(300 \mathrm{MHz}, \mathrm{CDCl}_{3}\right) \delta$ : $0.99(\mathrm{~d}, 18-\mathrm{H}), 1.10\left(\mathrm{~d}, 6^{\prime \prime}-\mathrm{H}\right), 1.15\left(\mathrm{~d}, 6^{\prime}-\mathrm{H}\right), 1.17$ (t, 3$\left.\mathrm{OCOCH}_{2} \mathrm{CH}_{3}\right), 1.20\left(\mathrm{t}, 4^{\prime \prime}-\mathrm{OCOCH}_{2} \mathrm{CH}_{3}\right), 1.28(\mathrm{~d}, 15-\mathrm{H})$, 1.42 (s, 3"- $\mathrm{CH}_{3}$ ), 1.56 (br dd, 7-H), 1.70 (dd, 2"-Hax), 1.88 (m, 13-H), 2.01 (s, 3"-OCOCH ${ }_{3}$ ), 2.07 (brt, 6-H), 2.34 (s, $\left.11-\mathrm{NCH}_{3}\right), 2.56\left(\mathrm{~s}, 3^{\prime}-\mathrm{N}\left(\mathrm{CH}_{3}\right)_{2}\right), 2.80(\mathrm{dd}, 2-\mathrm{H}), 2.96(\mathrm{dd}$, 16-H), 3.22 (m, 4'-H), 3.22 (m, 5'-H), 3.23 (d, 2"-Heq), $3.37\left(\mathrm{dd}, 2^{\prime}-\mathrm{H}\right), 3.60\left(\mathrm{~s}, 4-\mathrm{OCH}_{3}\right), 3.62(\mathrm{dd}, 4-\mathrm{H}), 3.87$ (br d, 5-H), 4.43 (d, 1'-H), 4.53 (dq, 5"-H), 4.59 (d, 4"-H), 4.86 (d, 1"-H), 4.88 (m, 14-H), 5.40 (br dd, 3-H), 9.66 (s, 17-H).

$(-)-(3 R, 4 S, 5 S, 6 R, 8 R, 9 R, 14 R)-9$-Acetoxy-5-[2-O-acetyl-4$O$-(3- $O$-acetyl-2,6-dideoxy-3- $C$-methyl-4- $O$-propionyl- $\alpha$-Lribo-hexopyranosyl)-3,6-dideoxy-3-dimethylamino- $\beta$-Dglucopyranosyloxy]-6-(2,2-dimethoxyethyl)-4-methoxy-8methyl-11-(2-phenylethyl)-3-propionyloxy-11-aza-pentadecan-14-olide (28a)

Reaction of $\mathbf{1 2}$ with 2-phenylethylamine gave 28a in 7.3\% yield by a similar procedure to $\mathbf{1 6}$.

28a: $[\alpha]_{\mathrm{D}}^{25}-86^{\circ}\left(c\right.$ 1.0, $\left.\mathrm{CHCl}_{3}\right)$; FAB-MS $m / z 1081$ $(\mathrm{M}+\mathrm{H})^{+}$as $\mathrm{C}_{55} \mathrm{H}_{88} \mathrm{~N}_{2} \mathrm{O}_{19} ;{ }^{1} \mathrm{H}-\mathrm{NMR}\left(300 \mathrm{MHz}, \mathrm{CDCl}_{3}\right) \delta$ : 0.93 (d, 18-H), 1.08 (d, 6"-H), 1.15 (t, 3-OCOCH $\mathrm{CH}_{3}$ ), 1.20 (t, 4"-OCOCH $\left.\mathrm{CH}_{3}\right), 1.21\left(\mathrm{~d}, 6^{\prime}-\mathrm{H}\right), 1.23(\mathrm{~d}, 15-\mathrm{H})$, 1.42 (s, 3"- $\left.\mathrm{CH}_{3}\right), 1.55$ (br dd, 16-H), 1.68 (dd, 2"-Hax), 1.82 (m, 16-H), $2.02\left(\mathrm{~s}, 9-\mathrm{OCOCH}_{3}\right), 2.03\left(\mathrm{~s}, 3^{\prime \prime}-\mathrm{OCOCH}_{3}\right)$, $2.05\left(\mathrm{~s}, 2^{\prime}-\mathrm{OCOCH}_{3}\right), 2.44\left(\mathrm{~s}, 3^{\prime}-\mathrm{N}\left(\mathrm{CH}_{3}\right)_{2}\right), 2.83(\mathrm{dd}, 2-\mathrm{H})$, $3.15\left(\mathrm{~s}, 17-\mathrm{OCH}_{3}\right), 3.20$ (d, 2"-Heq), $3.26\left(\mathrm{~s}, 17-\mathrm{OCH}_{3}\right)$, 3.43 (br d, 4-H), 3.56 (s, 4- $\mathrm{OCH}_{3}$ ), 3.90 (br d, 5-H), 4.50 (dq, 5"-H), 4.57 (d, 4"-H), 4.68 (d, 1'-H), 4.78 (m, 9-H), 4.82 (d, 1"-H), 4.94 (ddq, 14-H), 4.99 (dd, 2'-H), 5.17 (m, 3-H), $7.19\left(\mathrm{~m}, \mathrm{C}_{6} \mathrm{H}_{5}\right), 7.27\left(\mathrm{~m}, \mathrm{C}_{6} \mathrm{H}_{5}\right)$.

(-)-(3R,4S,5S,6R,8R,9R,14R)-9-Acetoxy-5-[4-O-(3-Oacetyl-2,6-dideoxy-3- $C$-methyl-4- $O$-propionyl- $\alpha$-L-ribohexopyranosyl)-3,6-dideoxy-3-dimethylamino- $\beta$-D-glucopyranosyloxy]-6-(2,2-dimethoxyethyl)-4-methoxy-8-methyl11-(2-phenylethyl)-3-propionyloxy-11-aza-pentadecan-14olide (29a) and (-)-(3R,4S,5S,6R,8R,9R,14R)-5-[4-O(3-O-Acetyl-2,6-dideoxy-3-C-methyl-4- $O$-propionyl- $\alpha$-Lribo-hexopyranosyl)-3,6-dideoxy-3-dimethylamino- $\beta$-Dglucopyranosyloxy]-6-(2,2-dimethoxyethyl)-9-hydroxy-4methoxy-8-methyl-11-(2-phenylethyl)-3-propionyloxy-11aza-pentadecan-14-olide (30a)

Reaction of 28a with methanol gave 29a in 54\% yield and $\mathbf{3 0 a}$ in $32 \%$ yield, respectively, by a similar procedure to $\mathbf{2 6}$. Total recovery was $86 \%$ in this reaction.

29a: $[\alpha]_{\mathrm{D}}^{24}-65^{\circ}\left(c\right.$ 1.0, $\mathrm{CHCl}_{3}$ ); FAB-MS $m / z 1039$ $(\mathrm{M}+\mathrm{H})^{+}$as $\mathrm{C}_{53} \mathrm{H}_{86} \mathrm{~N}_{2} \mathrm{O}_{18} ;{ }^{1} \mathrm{H}-\mathrm{NMR}\left(300 \mathrm{MHz}, \mathrm{CDCl}_{3}\right) \delta$ : 0.93 (d, 18-H), 1.10 (d, 6"-H), 1.15 (t, 3-OCOCH $\mathrm{CH}_{3}$ ), 1.20 (t, 4"-OCOCH$\left.{ }_{2} \mathrm{CH}_{3}\right), 1.22\left(\mathrm{~d}, 6^{\prime}-\mathrm{H}\right), 1.24(\mathrm{~d}, 15-\mathrm{H})$, 1.42 (s, 3"- $\left.\mathrm{CH}_{3}\right), 1.55$ (m, 16-H), 1.70 (dd, 2"-Hax), 2.03 (s, 9- $\left.\mathrm{OCOCH}_{3}\right), 2.03\left(\mathrm{~s}, 3^{\prime \prime}-\mathrm{OCOCH}_{3}\right), 2.56\left(\mathrm{~s}, 3^{\prime}-\mathrm{N}\left(\mathrm{CH}_{3}\right)_{2}\right)$, $2.85(\mathrm{dd}, 2-\mathrm{H}), 3.18\left(\mathrm{~s}, 17-\mathrm{OCH}_{3}\right), 3.20\left(\mathrm{~m}, 4^{\prime}-\mathrm{H}\right), 3.20(\mathrm{~m}$, $\left.5^{\prime}-\mathrm{H}\right), 3.23$ (d, 2'-Heq), 3.27 (s, 17-- $\mathrm{OCH}_{3}$ ), 3.44 (dd, 2'-H), 3.50 (br d, 4-H), $3.62\left(\mathrm{~s}, 4-\mathrm{OCH}_{3}\right), 3.91$ (br d, 5-H), 4.50 (d, $\left.1^{\prime}-\mathrm{H}\right), 4.54$ (dq, 5"-H), 4.59 (d, 4"-H), 4.81 (m, 9-H), 4.86 (d, 1"-H), 4.95 (m, 14-H), $5.23(\mathrm{~m}, 3-\mathrm{H}), 7.19$ (m, $\left.\mathrm{C}_{6} \mathrm{H}_{5}\right)$, $7.27\left(\mathrm{~m}, \mathrm{C}_{6} \mathrm{H}_{5}\right)$. 
30a: $[\alpha]_{\mathrm{D}}^{24}-67^{\circ}\left(c \quad 0.71, \mathrm{CHCl}_{3}\right)$; FAB-MS $\mathrm{m} / \mathrm{z} 997$ $(\mathrm{M}+\mathrm{H})^{+}$as $\mathrm{C}_{51} \mathrm{H}_{84} \mathrm{~N}_{2} \mathrm{O}_{17} ;{ }^{1} \mathrm{H}-\mathrm{NMR}\left(300 \mathrm{MHz}, \mathrm{CDCl}_{3}\right) \delta$ : 0.95 (d, 18-H), 1.10 (d, 6"-H), 1.13 (t, 3-OCOCH$\left.{ }_{2} \mathrm{CH}_{3}\right)$, $1.20\left(\mathrm{t}, 4^{\prime \prime}-\mathrm{OCOCH}_{2} \mathrm{CH}_{3}\right), 1.22\left(\mathrm{~d}, 6^{\prime}-\mathrm{H}\right), 1.24(\mathrm{~d}, 15-\mathrm{H})$, 1.40 (br dd, 7-H), 1.43 (s, 3"- $\left.\mathrm{CH}_{3}\right), 1.61$ (m, 8-H), 1.61 (m, 16-H), 1.70 (dd, 2"-Hax), 1.89 (ddd, 16-H), 2.02 (s, 3"$\left.\mathrm{OCOCH}_{3}\right), 2.40\left(\mathrm{t}, 3^{\prime}-\mathrm{H}\right), 2.55\left(\mathrm{~s}, 3^{\prime}-\mathrm{N}\left(\mathrm{CH}_{3}\right)_{2}\right), 2.59$ (dd, 2$\mathrm{H}), 2.82(\mathrm{dd}, 2-\mathrm{H}), 3.14\left(\mathrm{~s}, 17-\mathrm{OCH}_{3}\right), 3.22\left(\mathrm{~m}, 4^{\prime}-\mathrm{H}\right), 3.22$ (m, 5'-H), 3.23 (d, 2"-Heq), 3.27 (s, 17- $\left.\mathrm{OCH}_{3}\right), 3.41$ (dd, $\left.2^{\prime}-\mathrm{H}\right), 3.55$ (dd, 4-H), 3.60 (s, 4-- $\left.\mathrm{OCH}_{3}\right), 3.90$ (br d, 5-H), 4.48 (d, 1'-H), 4.50 (dd, 17-H), 4.55 (dq, 5"-H), 4.59 (d, 4" H), 4.77 (ddq, 14-H), 4.86 (d, 1"-H), 5.20 (br dd, 3-H), 7.20 $\left(\mathrm{m}, \mathrm{C}_{6} \mathrm{H}_{5}\right), 7.30\left(\mathrm{~m}, \mathrm{C}_{6} \mathrm{H}_{5}\right)$.

(-)-(3R,4S,5S,6R,8R,9R,14R)-9-Acetoxy-5-[4-O-(3-Oacetyl-2,6-dideoxy-3-C-methyl-4- $O$-propionyl- $\alpha$-L-ribohexopyranosyl)-3,6-dideoxy-3-dimethylamino- $\beta$-D-glucopyranosyloxy]-6-formylmethyl-4-methoxy-8-methyl-11(2-phenylethyl)-3-propionyloxy-11-aza-pentadecan-14olide (31a)

Reaction of 29a with aqueous difluoroacetic acid gave 31a in $95 \%$ yield by a similar procedure to 11 .

31a: $[\alpha]_{\mathrm{D}}^{23}-65^{\circ}\left(c \quad 0.98, \mathrm{CHCl}_{3}\right)$; FAB-MS $m / z 993$ $(\mathrm{M}+\mathrm{H})^{+}$as $\mathrm{C}_{51} \mathrm{H}_{80} \mathrm{~N}_{2} \mathrm{O}_{17} ;{ }^{1} \mathrm{H}-\mathrm{NMR}\left(300 \mathrm{MHz}, \mathrm{CDCl}_{3}\right) \delta$ : $0.93(\mathrm{~d}, 18-\mathrm{H}), 1.10\left(\mathrm{~d}, 6^{\prime \prime}-\mathrm{H}\right), 1.15\left(\mathrm{~d}, 6^{\prime}-\mathrm{H}\right), 1.18$ (t, 3$\mathrm{OCOCH}_{2} \mathrm{CH}_{3}$ ), 1.20 (t, 4"-OCOCH $\left.\mathrm{CH}_{3}\right), 1.27$ (d, 15-H), 1.42 (s, 3"- $\mathrm{CH}_{3}$ ), 1.48 (br dd, 7-H), 1.70 (dd, 2"-Hax), 2.02 $\left(\mathrm{s}, 9-\mathrm{OCOCH}_{3}\right), 2.02\left(\mathrm{~s}, 3^{\prime \prime}-\mathrm{OCOCH}_{3}\right), 2.20(\mathrm{~m}, 6-\mathrm{H}), 2.57$ (s, 3'-N( $\left.\left(\mathrm{CH}_{3}\right)_{2}\right), 2.95(\mathrm{dd}, 16-\mathrm{H}), 3.23\left(\mathrm{~m}, 4^{\prime}-\mathrm{H}\right), 3.23(\mathrm{~m}$, $\left.5^{\prime}-\mathrm{H}\right), 3.23$ (d, 2"'-Heq), 3.27 (dd, 2'-H), 3.58 (br d, 4-H), $3.60\left(\mathrm{~s}, 4-\mathrm{OCH}_{3}\right), 3.94$ (br d, 5-H), $4.46\left(\mathrm{~d}, 1^{\prime}-\mathrm{H}\right), 4.52$ (dq, 5"-H), 4.59 (d, 4"-H), 4.79 (m, 9-H), 4.87 (d, 1"'-H), 4.87 (m, 14-H), 5.37 (m, 3-H), $7.19\left(\mathrm{~m}, \mathrm{C}_{6} \mathrm{H}_{5}\right), 7.27\left(\mathrm{~m}, \mathrm{C}_{6} \mathrm{H}_{5}\right)$, $9.65(\mathrm{~s}, 17-\mathrm{H})$.

(-)-(3R,4S,5S,6R,8R,9R,14R)-5-[4-O-(3-O-Acetyl-2,6dideoxy-3-C-methyl-4- $O$-propionyl- $\alpha$-L-ribo-hexopyranosyl)-3,6-dideoxy-3-dimethylamino- $\beta$-D-glucopyranosyloxy]-6-formylmethyl-9-hydroxy-4-methoxy-8-methyl-11(2-phenylethyl)-3-propionyloxy-11-aza-pentadecan-14olide (32a)

Reaction of 30a with aqueous difluoroacetic acid gave 32a in $90 \%$ yield by a similar procedure to $\mathbf{1 1}$.

32a: $[\alpha]_{\mathrm{D}}^{22}-73^{\circ}\left(c \quad 0.60, \mathrm{CHCl}_{3}\right)$; FAB-MS $m / z 951$ $(\mathrm{M}+\mathrm{H})^{+}$as $\mathrm{C}_{49} \mathrm{H}_{78} \mathrm{~N}_{2} \mathrm{O}_{16} ;{ }^{1} \mathrm{H}-\mathrm{NMR}\left(300 \mathrm{MHz}, \mathrm{CDCl}_{3}\right) \delta$ : $0.96(\mathrm{~d}, 18-\mathrm{H}), 1.10\left(\mathrm{~d}, 6^{\prime \prime}-\mathrm{H}\right), 1.15\left(\mathrm{~d}, 6^{\prime}-\mathrm{H}\right), 1.17$ (t, 3$\left.\mathrm{OCOCH}_{2} \mathrm{CH}_{3}\right), 1.20$ (t, 4"-OCOCH $\left.\mathrm{CH}_{3}\right), 1.27$ (d, 15-H), 1.42 (s, 3"- $\left.\mathrm{CH}_{3}\right), 1.46$ (br dd, 7-H), 1.71 (dd, 2"-Hax), 2.01 (s, 3"- $\left.\mathrm{OCOCH}_{3}\right), 2.01(\mathrm{~m}, 6-\mathrm{H}), 2.56\left(\mathrm{~s}, 3^{\prime}-\mathrm{N}\left(\mathrm{CH}_{3}\right)_{2}\right), 2.60$ (dd, 2-H), 2.81 (dd, 2-H), 2.96 (dd, 16-H), 3.23 (m, 4'-H), 3.23 (m, 5'-H), 3.23 (d, 2"-Heq), 3.34 (dd, 2'-H), 3.60 (s, 4-
$\mathrm{OCH}_{3}$ ), 3.64 (br d, 4-H), 3.89 (br d, 5-H), 4.44 (d, 1'-H), 4.51 (dq, 5"-H), 4.59 (d, 4"-H), 4.76 (ddq, 14-H), 4.86 (d, 1"-H), 5.33 (br dd, 3-H), $7.20\left(\mathrm{~m}, \mathrm{C}_{6} \mathrm{H}_{5}\right), 7.30\left(\mathrm{~m}, \mathrm{C}_{6} \mathrm{H}_{5}\right)$, $9.65(\mathrm{~s}, 17-\mathrm{H})$.

$(-)-(3 R, 4 S, 5 S, 6 R, 8 R, 9 R, 14 R)-9$-Acetoxy-5-[2-O-acetyl-4$O$-(3-O-acetyl-2,6-dideoxy-3- $C$-methyl-4- $O$-propionyl- $\alpha$-Lribo-hexopyranosyl)-3,6-dideoxy-3-dimethylamino- $\beta$-Dglucopyranosyloxy]-6-(2,2-dimethoxyethyl)-4-methoxy-8methyl-11-(3-phenylpropyl)-3-propionyloxy-11-aza-pentadecan-14-olide (28b)

Reaction of $\mathbf{1 2}$ with 3-phenylpropylamine gave $\mathbf{2 8 b}$ in $7.3 \%$ yield by a similar procedure to $\mathbf{1 6}$.

28b: $[\alpha]_{\mathrm{D}}^{26}-86^{\circ}$ (c 1.0, $\mathrm{CHCl}_{3}$ ); FAB-MS $m / z 1095$ $(\mathrm{M}+\mathrm{H})^{+}$as $\mathrm{C}_{56} \mathrm{H}_{90} \mathrm{~N}_{2} \mathrm{O}_{19} ;{ }^{1} \mathrm{H}-\mathrm{NMR}\left(300 \mathrm{MHz}, \mathrm{CDCl}_{3}\right) \delta$ : $0.92(\mathrm{~d}, 18-\mathrm{H}), 1.07$ (d, 6"-H), 1.14 (t, 3-OCOCH$\left.{ }_{2} \mathrm{CH}_{3}\right)$, 1.19 (t, 4"-OCOCH$\left.{ }_{2} \mathrm{CH}_{3}\right), 1.20$ (d, 6'-H), 1.24 (d, 15-H), 1.42 (s, 3"- $-\mathrm{CH}_{3}$ ), 1.54 (br dd, 16-H), 1.67 (dd, 2"-Hax), 1.70 (m, $\left.\mathrm{Ph}\left(\mathrm{CH}_{2}\right)_{3}\right), 1.85(\mathrm{~m}, 16-\mathrm{H}), 1.99\left(\mathrm{~s}, 9-\mathrm{OCOCH}_{3}\right), 2.02$ $\left(\mathrm{s}, 3^{\prime \prime}-\mathrm{OCOCH}_{3}\right), 2.04\left(\mathrm{~s}, 2^{\prime}-\mathrm{OCOCH}_{3}\right), 2.44\left(\mathrm{~s}, 33^{\prime}-\right.$ $\left.\mathrm{N}\left(\mathrm{CH}_{3}\right)_{2}\right), 2.84$ (dd, 2-H), $3.14\left(\mathrm{~s}, 17-\mathrm{OCH}_{3}\right), 3.14$ (m, 4' H), 3.15 (d, 2"-Heq), 3.25 (s, 17--OCH $), 3.46$ (br d, 4-H), $3.55\left(\mathrm{~s}, 4-\mathrm{OCH}_{3}\right), 3.90$ (br d, 5-H), 4.49 (dq, 5"-H), 4.57 (d, 4"-H), 4.68 (d, 1'-H), 4.79 (m, 9-H), 4.81 (d, 1"'-H), 4.93 (m, 14-H), 4.98 (dd, 2'-H), $5.16(\mathrm{~m}, 3-\mathrm{H}), 7.17$ (m, $\mathrm{C}_{6} \mathrm{H}_{5}$ ), $7.27\left(\mathrm{~m}, \mathrm{C}_{6} \mathrm{H}_{5}\right)$.

(-)-(3R,4S,5S,6R,8R,9R,14R)-9-Acetoxy-5-[4-O-(3-Oacetyl-2,6-dideoxy-3-C-methyl-4- $O$-propionyl- $\alpha$-L-ribohexopyranosyl)-3,6-dideoxy-3-dimethylamino- $\beta$-D-glucopyranosyloxy]-6-(2,2-dimethoxyethyl)-4-methoxy-8-methyl11-(3-phenylpropyl)-3-propionyloxy-11-aza-pentadecan14-olide (29b) and (-)-(3R,4S,5S,6R,8R,9R,14R)-5-[4-O(3-O-Acetyl-2,6-dideoxy-3- $C$-methyl-4- $O$-propionyl- $\alpha$-Lribo-hexopyranosyl)-3,6-dideoxy-3-dimethylamino- $\beta$-Dglucopyranosyloxy]-6-(2,2-dimethoxyethyl)-9-hydroxy-4methoxy-8-methyl-11-(3-phenylpropyl)-3-propionyloxy11-aza-pentadecan-14-olide (30b)

Reaction of $\mathbf{2 8 b}$ with methanol gave $\mathbf{2 9 b}$ in $38 \%$ yield and 30b in 33\% yield, respectively, by a similar procedure to 26. Total recovery was $71 \%$ in this reaction.

29b: $[\alpha]_{\mathrm{D}}^{24}-58^{\circ}\left(c 0.90, \mathrm{CHCl}_{3}\right)$; FAB-MS $m / z 1053$ $(\mathrm{M}+\mathrm{H})^{+}$as $\mathrm{C}_{54} \mathrm{H}_{88} \mathrm{~N}_{2} \mathrm{O}_{18} ;{ }^{1} \mathrm{H}-\mathrm{NMR}\left(300 \mathrm{MHz}, \mathrm{CDCl}_{3}\right) \delta$ : $0.96(\mathrm{~d}, 18-\mathrm{H}), 1.10\left(\mathrm{~d}, 6^{\prime \prime}-\mathrm{H}\right), 1.13\left(\mathrm{t}, 3-\mathrm{OCOCH}_{2} \mathrm{CH}_{3}\right)$, 1.20 (t, 4"-OCOCH$\left.{ }_{2} \mathrm{CH}_{3}\right), 1.22\left(\mathrm{~d}, 6^{\prime}-\mathrm{H}\right), 1.26(\mathrm{~d}, 15-\mathrm{H})$, $1.43\left(\mathrm{~s}, 3^{\prime \prime}-\mathrm{CH}_{3}\right), 1.44$ (br dd, 7-H), 1.60 (m, 8-H), 1.60 (m, 16-H), 1.70 (dd, 2"-Hax), 1.86 (m, $\left.\mathrm{Ph}\left(\mathrm{CH}_{2}\right)_{3}\right), 1.90$ (m, 16$\mathrm{H}), 2.02\left(\mathrm{~s}, 3^{\prime \prime}-\mathrm{OCOCH}_{3}\right), 2.55\left(\mathrm{~s}, 3^{\prime}-\mathrm{N}\left(\mathrm{CH}_{3}\right)_{2}\right), 2.83(\mathrm{dd}, 2-$ $\mathrm{H}), 3.13\left(\mathrm{~s}, 17-\mathrm{OCH}_{3}\right), 3.22\left(\mathrm{~m}, 4^{\prime}-\mathrm{H}\right), 3.22\left(\mathrm{~m}, 5^{\prime}-\mathrm{H}\right), 3.23$ (d, 2"-Heq), 3.27 (s, 17- $-\mathrm{OCH}_{3}$ ), 3.41 (dd, 2'-H), 3.58 (br d, 4-H), 3.60 (s, 4- $\left.\mathrm{OCH}_{3}\right), 3.90($ br d, 5-H), 4.49 (d, 1'-H), 
4.52 (dq, 5"-H), 4.59 (d, 4"-H), 4.83 (ddq, 14-H), 4.86 (d, 1"-H), 5.22 (br dd, 3-H), $7.19\left(\mathrm{~m}, \mathrm{C}_{6} \mathrm{H}_{5}\right), 7.29\left(\mathrm{~m}, \mathrm{C}_{6} \mathrm{H}_{5}\right)$.

30b: $[\alpha]_{\mathrm{D}}^{26}-60^{\circ}\left(c \quad 0.76, \mathrm{CHCl}_{3}\right)$; FAB-MS $m / z 1011$ $(\mathrm{M}+\mathrm{H})^{+}$as $\mathrm{C}_{52} \mathrm{H}_{86} \mathrm{~N}_{2} \mathrm{O}_{17} ;{ }^{1} \mathrm{H}-\mathrm{NMR}\left(300 \mathrm{MHz}, \mathrm{CDCl}_{3}\right) \delta$ : $0.96(\mathrm{~d}, 18-\mathrm{H}), 1.10\left(\mathrm{~d}, 66^{\prime \prime}-\mathrm{H}\right), 1.13\left(\mathrm{t}, 3-\mathrm{OCOCH}_{2} \mathrm{CH}_{3}\right)$, $1.20\left(\mathrm{t}, 4^{\prime \prime}-\mathrm{OCOCH}_{2} \mathrm{CH}_{3}\right), 1.22\left(\mathrm{~d}, 6^{\prime}-\mathrm{H}\right), 1.26(\mathrm{~d}, 15-\mathrm{H})$, $1.43\left(\mathrm{~s}, 3^{\prime \prime}-\mathrm{CH}_{3}\right), 1.44$ (br dd, 7-H), $1.60(\mathrm{~m}, 8-\mathrm{H}), 1.60$ (m, 16-H), 1.70 (dd, 2"-Hax), 1.86 (m, $\left.\mathrm{Ph}\left(\mathrm{CH}_{2}\right)_{3}\right), 1.90$ (m, 16$\mathrm{H}), 2.02\left(\mathrm{~s}, 3^{\prime \prime}-\mathrm{OCOCH}_{3}\right), 2.55\left(\mathrm{~s}, 3^{\prime}-\mathrm{N}\left(\mathrm{CH}_{3}\right)_{2}\right), 2.83(\mathrm{dd}, 2-$ $\mathrm{H}), 3.13\left(\mathrm{~s}, 17-\mathrm{OCH}_{3}\right), 3.22\left(\mathrm{~m}, 4^{\prime}-\mathrm{H}\right), 3.22\left(\mathrm{~m}, 5^{\prime}-\mathrm{H}\right), 3.23$ (d, 2"-Heq), 3.27 (s, 17- $\left.\mathrm{OCH}_{3}\right), 3.41$ (dd, 2'-H), 3.58 (br d, 4-H), 3.60 (s, 4-- $\mathrm{OCH}_{3}$ ), 3.90 (br d, 5-H), 4.49 (d, 1'-H), 4.52 (dq, 5"-H), 4.59 (d, 4"-H), 4.83 (ddq, 14-H), 4.86 (d, 1"-H), 5.22 (br dd, 3-H), $7.19\left(\mathrm{~m}, \mathrm{C}_{6} \mathrm{H}_{5}\right), 7.29\left(\mathrm{~m}, \mathrm{C}_{6} \mathrm{H}_{5}\right)$.

(-)-(3R,4S,5S,6R,8R,9R,14R)-9-Acetoxy-5-[4-O-(3-Oacetyl-2,6-dideoxy-3- $C$-methyl-4- $O$-propionyl- $\alpha$-L-ribohexopyranosyl)-3,6-dideoxy-3-dimethylamino- $\beta$-Dglucopyranosyloxy]-6-formylmethyl-4-methoxy-8-methyl11-(3-phenylpropyl)-3-propionyloxy-11-aza-pentadecan14-olide (31b)

Reaction of 29b with aqueous difluoroacetic acid gave 31b in $70 \%$ yield by a similar procedure to $\mathbf{1 1}$.

31b: $[\alpha]_{\mathrm{D}}^{25}-70^{\circ}\left(c 0.61, \mathrm{CHCl}_{3}\right)$; FAB-MS $m / z 1007$ $(\mathrm{M}+\mathrm{H})^{+}$as $\mathrm{C}_{52} \mathrm{H}_{82} \mathrm{~N}_{2} \mathrm{O}_{17} ;{ }^{1} \mathrm{H}-\mathrm{NMR}\left(300 \mathrm{MHz}, \mathrm{CDCl}_{3}\right) \delta$ : 0.93 (d, 18-H), 1.10 (d, 6"-H), 1.16 (d, 6'-H), 1.18 (t, 3$\left.\mathrm{OCOCH}_{2} \mathrm{CH}_{3}\right), 1.20\left(\mathrm{t}, 4^{\prime \prime}-\mathrm{OCOCH}_{2} \mathrm{CH}_{3}\right), 1.26(\mathrm{~d}, 15-\mathrm{H})$, 1.43 (s, 3"- $\mathrm{CH}_{3}$ ), 1.45 (br dd, 7-H), 1.71 (dd, 2"-Hax), 2.00 (s, 9-OCOCH 3 ), $2.02\left(\mathrm{~s}, 3^{\prime \prime}-\mathrm{OCOCH}_{3}\right), 2.20(\mathrm{~m}, 6-\mathrm{H}), 2.57$ (s, 3'-N( $\left.\left.\mathrm{CH}_{3}\right)_{2}\right), 2.84(\mathrm{dd}, 2-\mathrm{H}), 2.95(\mathrm{dd}, 16-\mathrm{H}), 3.23(\mathrm{~m}$, $\left.4^{\prime}-\mathrm{H}\right), 3.23$ (m, 5'-H), 3.23 (d, 2'-Heq), 3.26 (dd, 2'-H), $3.59\left(\mathrm{~s}, 4-\mathrm{OCH}_{3}\right), 3.94$ (dd, 5-H), 4.46 (d, 1'-H), 4.49 (dq, 5"-H), 4.60 (d, 4"-H), 4.79 (m, 14-H), 4.82 (m, 9-H), 4.87 (d, 1"-H), $5.36(\mathrm{~m}, 3-\mathrm{H}), 7.18\left(\mathrm{~m}, \mathrm{C}_{6} \mathrm{H}_{5}\right), 7.28\left(\mathrm{~m}, \mathrm{C}_{6} \mathrm{H}_{5}\right)$, $9.64(\mathrm{~s}, 17-\mathrm{H})$.

(-)-(3R,4S,5S,6R,8R,9R,14R)-5-[4-O-(3-O-Acetyl-2,6dideoxy-3-C-methyl-4- $O$-propionyl- $\alpha$-L-ribo-hexopyranosyl)-3,6-dideoxy-3-dimethylamino- $\beta$-D-glucopyranosyloxy]-6-formylmethyl-9-hydroxy-4-methoxy-8-methyl-11(3-phenylpropyl)-3-propionyloxy-11-aza-pentadecan-14olide (32b)

Reaction of 30b with aqueous difluoroacetic acid gave 32b in $84 \%$ yield by a similar procedure to 11 .

32b: $[\alpha]_{\mathrm{D}}^{26}-72^{\circ}\left(c 0.61, \mathrm{CHCl}_{3}\right)$; FAB-MS m/z 965 $(\mathrm{M}+\mathrm{H})^{+}$as $\mathrm{C}_{50} \mathrm{H}_{80} \mathrm{~N}_{2} \mathrm{O}_{16} ;{ }^{1} \mathrm{H}-\mathrm{NMR}\left(300 \mathrm{MHz}, \mathrm{CDCl}_{3}\right) \delta$ : 0.98 (d, 18-H), 1.10 (d, 6"-H), 1.15 (d, 6'-H), 1.17 (t, 3$\left.\mathrm{OCOCH}_{2} \mathrm{CH}_{3}\right), 1.20\left(\mathrm{t}, 4^{\prime \prime}-\mathrm{OCOCH} \mathrm{CH}_{3}\right), 1.27(\mathrm{~d}, 15-\mathrm{H})$, 1.43 (s, 3"- $\left.\mathrm{CH}_{3}\right), 1.47$ (m, 8-H), 1.71 (dd, 2"-Hax), 2.01 (s, 3"- $\left.-\mathrm{OCOCH}_{3}\right), 2.56\left(\mathrm{~s}, 3^{\prime}-\mathrm{N}\left(\mathrm{CH}_{3}\right)_{2}\right), 2.63\left(\mathrm{t}, \mathrm{Ph}\left(\mathrm{CH}_{2}\right)_{3}\right), 2.82$ (dd, 2-H), 2.97 (dd, 16-H), 3.23 (m, 4'-H), 3.23 (m, 5'-H),
3.27 (d, 2"-Heq), 3.53 (dd, 2'-H), 3.60 (s, 4- $\left.\mathrm{OCH}_{3}\right), 3.65$ (dd, 4-H), 3.89 (br d, 5-H), 4.44 (d, 1'-H), 4.50 (dq, 5"-H), 4.60 (d, 4"-H), 4.79 (ddq, 14-H), 4.86 (d, 1"-H), 5.34 (br dd, 3-H), $7.19\left(\mathrm{~m}, \mathrm{C}_{6} \mathrm{H}_{5}\right), 7.29\left(\mathrm{~m}, \mathrm{C}_{6} \mathrm{H}_{5}\right), 9.66(\mathrm{~s}, 17-\mathrm{H})$.

$(-)-(3 R, 4 S, 5 S, 6 R, 8 R, 9 R, 14 R)-9$-Acetoxy-5-[2-O-acetyl-4$O$-(3- $O$-acetyl-2,6-dideoxy-3- $C$-methyl-4- $O$-propionyl- $\alpha$-Lribo-hexopyranosyl)-3,6-dideoxy-3-dimethylamino- $\beta$-Dglucopyranosyloxy]-6-(2,2-dimethoxyethyl)-4-methoxy-8methyl-11-(4-phenylbutyl)-3-propionyloxy-11-aza-pentadecan-14-olide (28c)

Reaction of $\mathbf{1 2}$ with 4-phenylbutylamine gave $\mathbf{2 8 c}$ in $7.7 \%$ yield by a similar procedure to $\mathbf{1 6}$.

28c: $[\alpha]_{\mathrm{D}}^{23}-69^{\circ}\left(c \quad 0.53, \mathrm{CHCl}_{3}\right)$; FAB-MS $m / z 1109$ $(\mathrm{M}+\mathrm{H})^{+}$as $\mathrm{C}_{57} \mathrm{H}_{92} \mathrm{~N}_{2} \mathrm{O}_{19} ;{ }^{1} \mathrm{H}-\mathrm{NMR}\left(300 \mathrm{MHz}, \mathrm{CDCl}_{3}\right) \delta$ : 0.92 (d, 18-H), 1.08 (d, 6"-H), 1.14 (t, 3-OCOCH${ }_{2} \mathrm{CH}_{3}$ ), 1.20 (t, 4"-OCOCH$\left.{ }_{2} \mathrm{CH}_{3}\right), 1.21\left(\mathrm{~d}, 6^{\prime}-\mathrm{H}\right), 1.25(\mathrm{~d}, 15-\mathrm{H})$, 1.42 (s, 3"- $\left.\mathrm{CH}_{3}\right), 1.58$ (m, 16-H), 1.68 (dd, 2"-Hax), 1.81 (m, 16-H), 1.99 (s, 9-OCOCH $\left.{ }_{3}\right), 2.02\left(\mathrm{~s}, 3^{\prime \prime}-\mathrm{OCOCH}_{3}\right)$, $2.05\left(\mathrm{~s}, 2^{\prime}-\mathrm{OCOCH}_{3}\right), 2.44\left(\mathrm{~s}, 3^{\prime}-\mathrm{N}\left(\mathrm{CH}_{3}\right)_{2}\right), 2.83(\mathrm{dd}, 2-\mathrm{H})$, 3.14 (s, 17- $\left.\mathrm{OCH}_{3}\right), 3.20$ (d, 2"-Heq), 3.26 (s, 17- $\mathrm{OCH}_{3}$ ), 3.45 (br d, 4-H), 3.55 (s, 4- $\mathrm{OCH}_{3}$ ), 3.89 (br d, 5-H), 4.50 (dq, 5"-H), 4.57 (d, 4"-H), 4.68 (d, 1'-H), 4.78 (m, 9-H), 4.81 (d, 1"-H), 4.94 (m, 14-H), 4.98 (dd, 2'-H), 5.16 (m, 3$\mathrm{H}), 7.18\left(\mathrm{~m}, \mathrm{C}_{6} \mathrm{H}_{5}\right), 7.27\left(\mathrm{~m}, \mathrm{C}_{6} \mathrm{H}_{5}\right)$.

(-)- $(3 R, 4 S, 5 S, 6 R, 8 R, 9 R, 14 R)-9$-Acetoxy-5-[4- $O-(3-O-$ acetyl-2,6-dideoxy-3- $C$-methyl-4- $O$-propionyl- $\alpha$-L-ribohexopyranosyl)-3,6-dideoxy-3-dimethylamino- $\beta$-D-glucopyranosyl]-6-(2,2-dimethoxyethyl)-4-methoxy-8-methyl11-(4-phenylbutyl)-3-propionyloxy-11-aza-pentadecan-14olide (29c)

Reaction of $28 \mathrm{c}$ with methanol gave $29 \mathrm{c}$ in $14 \%$ yield by a similar procedure to $\mathbf{2 6}$.

29c: $[\alpha]_{\mathrm{D}}^{27}-65^{\circ}\left(c 0.61, \mathrm{CHCl}_{3}\right)$; FAB-MS $m / z 1067$ $(\mathrm{M}+\mathrm{H})^{+}$as $\mathrm{C}_{55} \mathrm{H}_{90} \mathrm{~N}_{2} \mathrm{O}_{18} ;{ }^{1} \mathrm{H}-\mathrm{NMR}\left(300 \mathrm{MHz}, \mathrm{CDCl}_{3}\right) \delta$ : 0.90 (d, 18-H), 1.07 (d, 6"-H), 1.12 (t, 3- $\left.\mathrm{OCOCH}_{2} \mathrm{CH}_{3}\right)$, 1.17 (t, 4"-OCOCH$\left.{ }_{2} \mathrm{CH}_{3}\right), 1.19$ (d, 6'-H), 1.23 (d, 15-H), $1.40\left(\mathrm{~s}, 3^{\prime \prime}-\mathrm{CH}_{3}\right), 1.68$ (dd, 2"-Hax), 1.97 (s, 9-- $\left.\mathrm{OCOCH}_{3}\right)$, $2.00\left(\mathrm{~s}, 3^{\prime \prime}-\mathrm{OCOCH}_{3}\right), 2.53\left(\mathrm{~s}, 3^{\prime}-\mathrm{N}\left(\mathrm{CH}_{3}\right)_{2}\right), 2.59$ (t, $\left.\mathrm{Ph}\left(\mathrm{CH}_{2}\right)_{4}\right), 2.83$ (dd, 2-H), $3.15\left(\mathrm{~s}, 17-\mathrm{OCH}_{3}\right), 3.21$ (d, 2"Heq), 3.25 (s, 17- $\mathrm{OCH}_{3}$ ), 3.41 (dd, 2'-H), 3.51 (br d, 4-H), 3.59 (s, 4- $\left.\mathrm{OCH}_{3}\right), 3.88$ (br d, 5-H), $4.47\left(\mathrm{~d}, \mathrm{1}^{\prime}-\mathrm{H}\right), 4.57$ (d, 4"-H), 4.77 (m, 9-H), 4.84 (d, 1"-H), 4.91 (m, 14-H), 5.20 (m, 3-H), $7.15\left(\mathrm{~m}, \mathrm{C}_{6} \mathrm{H}_{5}\right), 7.25\left(\mathrm{~m}, \mathrm{C}_{6} \mathrm{H}_{5}\right)$.

(-)-(3R,4S,5S,6R,8R,9R,14R)-9-Acetoxy-5-[4-O-(3-Oacetyl-2,6-dideoxy-3-C-methyl-4- $O$-propionyl- $\alpha$-L-ribohexopyranosyl)-3,6-dideoxy-3-dimethylamino- $\beta$-D-glucopyranosyloxy]-6-formylmethyl-4-methoxy-8-methyl-11(4-phenylbutyl)-3-propionyloxy-11-aza-pentadecan-14- 
olide (31c)

Reaction of 29c with aqueous difluoroacetic acid gave 31c in $51 \%$ yield by a similar procedure to 11 .

31c: $[\alpha]_{\mathrm{D}}^{26}-60^{\circ}\left(c 1.55, \mathrm{CHCl}_{3}\right)$; FAB-MS $m / z 1021$ $(\mathrm{M}+\mathrm{H})^{+}$as $\mathrm{C}_{53} \mathrm{H}_{84} \mathrm{~N}_{2} \mathrm{O}_{17} ;{ }^{1} \mathrm{H}-\mathrm{NMR}\left(300 \mathrm{MHz}, \mathrm{CDCl}_{3}\right) \delta$ : $0.91(\mathrm{~d}, 18-\mathrm{H}), 1.08\left(\mathrm{~d}, 6^{\prime \prime}-\mathrm{H}\right), 1.13\left(\mathrm{~d}, 6^{\prime}-\mathrm{H}\right), 1.16$ (t, 3$\left.\mathrm{OCOCH}_{2} \mathrm{CH}_{3}\right), 1.18\left(\mathrm{t}, 4^{\prime \prime}-\mathrm{OCOCH} \mathrm{CH}_{3}\right), 1.25(\mathrm{~d}, 15-\mathrm{H})$, 1.40 (s, 3"- $\left.\mathrm{CH}_{3}\right), 1.69$ (dd, 2"-Hax), 1.93 (s, 9-OCOCH ${ }_{3}$ ), 2.00 (s, 3"- $\left.-\mathrm{OCOCH}_{3}\right), 2.16(\mathrm{~m}, 6-\mathrm{H}), 2.54\left(\mathrm{~s}, 3^{\prime}-\mathrm{N}\left(\mathrm{CH}_{3}\right)_{2}\right)$, 2.81 (dd, 2-H), 2.93 (dd, 16-H), 3.21 (m, 4'-H), 3.21 (m, 5'H), 3.21 (d, 2"-Heq), 3.34 (dd, 2'-H), 3.58 (s, 4- $\left.\mathrm{OCH}_{3}\right)$, 3.91 (br d, 5-H), 4.44 (d, 1'-H), 4.49 (dq, 5"-H), 4.57 (d, 4"H), 4.75 (br dd, 9-H), 4.82 (m, 14-H), 4.84 (d, 1"-H), 5.34 (m, 3-H), $7.16\left(\mathrm{~m}, \mathrm{C}_{6} \mathrm{H}_{5}\right), 7.25\left(\mathrm{~m}, \mathrm{C}_{6} \mathrm{H}_{5}\right), 9.62(\mathrm{~s}, 17-\mathrm{H})$.

(-)-(3R,4S,5S,6R,8R,9R,14R)-5-[4-O-(3-O-Acetyl-2,6dideoxy-3-C-methyl-4- $O$-propionyl- $\alpha$-L-ribo-hexopyranosyl)-3,6-dideoxy-3-dimethylamino- $\beta$-D-glucopyranosyloxy]-6-(2,2-dimethoxyethyl)-9-hydroxy-4-methoxy-8methyl-11-(4-phenylbutyl)-3-propionyloxy-11-aza-pentadecan-14-olide (30c)

Reaction of 28c with methanol gave $30 \mathrm{c}$ in $62 \%$ yield by a similar procedure to $\mathbf{2 6}$.

30c: $[\alpha]_{\mathrm{D}}^{21}-68^{\circ}\left(c 0.60, \mathrm{CHCl}_{3}\right)$; FAB-MS $m / z 1025$ $(\mathrm{M}+\mathrm{H})^{+}$as $\mathrm{C}_{53} \mathrm{H}_{88} \mathrm{~N}_{2} \mathrm{O}_{17} ;{ }^{1} \mathrm{H}-\mathrm{NMR}\left(300 \mathrm{MHz}, \mathrm{CDCl}_{3}\right) \delta$ : 0.95 (d, 18-H), 1.07 (d, 6"-H), 1.11 (t, 3-OCOCH$\left.{ }_{2} \mathrm{CH}_{3}\right)$, 1.18 (t, 4"-OCOCH${ }_{2} \mathrm{CH}_{3}$ ), 1.19 (d, 6'-H), 1.24 (d, 15-H), 1.40 (s, 3"-- $\mathrm{CH}_{3}$ ), 1.68 (dd, 2"-Hax), 1.87 (br dd, 16-H), 2.00 (s, 3"-OCOCH$\left.{ }_{3}\right), 2.53\left(\mathrm{~s}, 3^{\prime}-\mathrm{N}\left(\mathrm{CH}_{3}\right)_{2}\right), 2.79$ (dd, 2-H), 3.11 (s, 17- $\left.\mathrm{OCH}_{3}\right), 3.21$ (d, 2"-Heq), 3.24 (s, 17- $\left.\mathrm{OCH}_{3}\right), 3.38$ (dd, 2'-H), 3.54 (br d, 4-H), 3.58 (s, 4-OCH ${ }_{3}$ ), 3.87 (br d, 5H), 4.47 (d, 1'-H), 4.51 (dq, 5"'-H), 4.57 (d, 4"'-H), 4.78 (m, 14-H), 4.84 (d, 1"-H), 5.20 (br dd, 3-H), $7.16\left(\mathrm{~m}, \mathrm{C}_{6} \mathrm{H}_{5}\right.$ ), $7.26\left(\mathrm{~m}, \mathrm{C}_{6} \mathrm{H}_{5}\right)$.

(-)-(3R,4S,5S,6R,8R,9R,14R)-5-[4-O-(3-O-Acetyl-2,6dideoxy-3-C-methyl-4- $O$-propionyl- $\alpha$-L-ribo-hexopyranosyl)-3,6-dideoxy-3-dimethylamino- $\beta$-D-glucopyranosyloxy]-6-formylmethyl-9-hydroxy-4-methoxy-8-methyl-11(4-phenylbutyl)-3-propionyloxy-11-aza-pentadecan-14olide (32c)

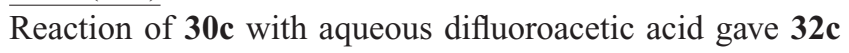
in $87 \%$ yield by a similar procedure to $\mathbf{1 1}$.

32c: $[\alpha]_{\mathrm{D}}^{22}-72^{\circ}\left(c 0.75, \mathrm{CHCl}_{3}\right)$; FAB-MS m/z 979 $(\mathrm{M}+\mathrm{H})^{+}$as $\mathrm{C}_{51} \mathrm{H}_{82} \mathrm{~N}_{2} \mathrm{O}_{16} ;{ }^{1} \mathrm{H}-\mathrm{NMR}\left(300 \mathrm{MHz}, \mathrm{CDCl}_{3}\right) \delta$ : 0.98 (d, 18-H), 1.10 (d, 6"-H), 1.15 (d, 6'-H), 1.17 (t, 3$\left.\mathrm{OCOCH}_{2} \mathrm{CH}_{3}\right), 1.20$ (t, 4"-OCOCH$\left.{ }_{2} \mathrm{CH}_{3}\right), 1.27(\mathrm{~d}, 15-\mathrm{H})$, 1.42 (s, 3"- $\left.-\mathrm{CH}_{3}\right), 1.49$ (m, $\left.\mathrm{Ph}\left(\mathrm{CH}_{2}\right)_{4}\right), 1.64$ (quint, $\left.\mathrm{Ph}\left(\mathrm{CH}_{2}\right)_{4}\right), 1.70$ (dd, 2"-Hax), 2.01 (s, 3"-OCOCH$\left.)_{3}\right), 2.36$ (dd, 16-H), $2.56\left(\mathrm{~s}, 3^{\prime}-\mathrm{N}\left(\mathrm{CH}_{3}\right)_{2}\right), 2.63\left(\mathrm{t}, \mathrm{Ph}\left(\mathrm{CH}_{2}\right)_{4}\right), 2.81$ (dd, 2-H), 2.97 (dd, 16-H), $3.22\left(\mathrm{~m}, 4^{\prime}-\mathrm{H}\right), 3.22\left(\mathrm{~m}, 5^{\prime}-\mathrm{H}\right)$,
3.23 (d, 2"-Heq), 3.34 (dd, 2'-H), $3.60\left(\mathrm{~s}, 4-\mathrm{OCH}_{3}\right), 3.65$ (dd, 4-H), 3.89 (br d, 5-H), 4.44 (d, 1'-H), 4.51 (dq, 5"-H), 4.59 (d, 4"-H), 4.78 (br dq, 14-H), 4.86 (d, 1"-H), 5.34 (brdd, 3-H), 7.19 (m, $\left.\mathrm{C}_{6} \mathrm{H}_{5}\right), 7.28\left(\mathrm{~m}, \mathrm{C}_{6} \mathrm{H}_{5}\right), 9.65$ (s, 17H).

(-)-(3R,4S,5S,6R,8R,9R,14R)-9-Acetoxy-5-[2-O-acetyl-4$O$-(3-O-acetyl-2,6-dideoxy-3- $C$-methyl-4- $O$-propionyl- $\alpha$-Lribo-hexopyranosyl)-3,6-dideoxy-3-dimethylamino- $\beta$-Dglucopyranosyloxy]-6-(2,2-dimethoxyethyl)-4-methoxy-8methyl-11-(5-phenylpentyl)-3-propionyloxy-11-aza-pentadecan-14-olide (28d)

Reaction of $\mathbf{1 2}$ with 5-phenylpentylamine gave $\mathbf{2 8 d}$ in $4.6 \%$ yield by a similar procedure to $\mathbf{1 6}$.

28d: $[\alpha]_{\mathrm{D}}^{26}-87^{\circ}\left(c 0.50, \mathrm{CHCl}_{3}\right)$; FAB-MS $m / z 1123$ $(\mathrm{M}+\mathrm{H})^{+}$as $\mathrm{C}_{58} \mathrm{H}_{94} \mathrm{~N}_{2} \mathrm{O}_{19} ;{ }^{1} \mathrm{H}-\mathrm{NMR}\left(300 \mathrm{MHz}, \mathrm{CDCl}_{3}\right) \delta$ : $0.92(\mathrm{~d}, 18-\mathrm{H}), 1.07$ (d, 6"-H), $1.14\left(\mathrm{t}, 3-\mathrm{OCOCH}_{2} \mathrm{CH}_{3}\right.$ ), 1.19 (t, 4"-OCOCH $\left.\mathrm{CH}_{3}\right), 1.20\left(\mathrm{~d}, 6^{\prime}-\mathrm{H}\right), 1.26(\mathrm{~d}, 15-\mathrm{H})$, 1.42 (s, 3"- $\left.\mathrm{CH}_{3}\right), 1.54$ (br dd, 16-H), 1.74 (dd, 2"-Hax), 1.82 $(\mathrm{m}, 16-\mathrm{H}), 2.00\left(\mathrm{~s}, 9-\mathrm{OCOCH}_{3}\right), 2.02\left(\mathrm{~s}, 3^{\prime \prime}-\mathrm{OCOCH}_{3}\right)$, $2.05\left(\mathrm{~s}, 2^{\prime}-\mathrm{OCOCH}_{3}\right), 2.44\left(\mathrm{~s}, 3^{\prime}-\mathrm{N}\left(\mathrm{CH}_{3}\right)_{2}\right), 2.60$ (t, $\left.\mathrm{Ph}\left(\mathrm{CH}_{2}\right)_{5}\right), 2.83$ (dd, 2-H), 3.14 (s, 17- $\left.\mathrm{OCH}_{3}\right), 3.16$ (m, 4'$\mathrm{H}), 3.19$ (d, 2"-Heq), 3.25 (m, 5'-H), 3.25 (s, 17- $\left.\mathrm{OCH}_{3}\right)$, 3.45 (br d, 4-H), 3.55 (s, 4-- $\mathrm{OCH}_{3}$ ), 3.89 (br d, 5-H), 4.49 (dq, 5"-H), 4.57 (d, 4"-H), 4.68 (d, 1'-H), 4.76 (m, 9-H), 4.81 (d, 1'-H), 4.95 (m, 14-H), 4.98 (dd, 2'-H), 5.16 (m, 3$\mathrm{H}), 7.17\left(\mathrm{~m}, \mathrm{C}_{6} \mathrm{H}_{5}\right), 7.27\left(\mathrm{~m}, \mathrm{C}_{6} \mathrm{H}_{5}\right)$.

(-)-(3R,4S,5S,6R,8R,9R,14R)-5-[4-O-(3-O-Acetyl-2,6dideoxy-3-C-methyl-4- $O$-propionyl- $\alpha$-L-ribo-hexopyranosyl)-3,6-dideoxy-3-dimethylamino- $\beta$-D-glucopyransyloxy]-6-(2,2-dimethoxyethyl)-9-hydroxy-4-methoxy-8methyl-11-(5-phenylpentyl)-3-propionyloxy-11-aza-pentadecan-14-olide (30d)

Reaction of 28d with methanol gave $\mathbf{3 0 d}$ in $43 \%$ yield by a similar procedure to $\mathbf{2 6}$.

30d: $[\alpha]_{\mathrm{D}}^{26}-66^{\circ}\left(c 1.30, \mathrm{CHCl}_{3}\right)$; FAB-MS $m / z 1039$ $(\mathrm{M}+\mathrm{H})^{+}$as $\mathrm{C}_{54} \mathrm{H}_{90} \mathrm{~N}_{2} \mathrm{O}_{17} ;{ }^{1} \mathrm{H}-\mathrm{NMR}\left(300 \mathrm{MHz}, \mathrm{CDCl}_{3}\right) \delta$ : $0.96(\mathrm{~d}, 18-\mathrm{H}), 1.09$ (d, 6"-H), 1.13 (t, 3-OCOCH $\mathrm{CH}_{3}$ ), $1.20\left(\mathrm{t}, 4^{\prime \prime}-\mathrm{OCOCH}_{2} \mathrm{CH}_{3}\right), 1.21\left(\mathrm{~d}, 6^{\prime}-\mathrm{H}\right), 1.27(\mathrm{~d}, 15-\mathrm{H})$, $1.42\left(\mathrm{~s}, 3^{\prime \prime}-\mathrm{CH}_{3}\right), 1.63$ (m, Ph( $\left.\left(\mathrm{CH}_{2}\right)_{5}\right), 1.70$ (dd, 2"-Hax), 1.89 (brdd, 16-H), 2.02 (s, 3"-OCOCH $\left.{ }_{3}\right), 2.55$ (s, 3'$\left.\mathrm{N}\left(\mathrm{CH}_{3}\right)_{2}\right), 2.61\left(\mathrm{~m}, \mathrm{Ph}\left(\mathrm{CH}_{2}\right)_{5}\right), 2.82(\mathrm{dd}, 2-\mathrm{H}), 3.14$ (s, 17$\left.\mathrm{OCH}_{3}\right), 3.21$ (m, 4'-H), 3.21 (m, 5'-H), 3.23 (d, 2"-Heq), $3.27\left(\mathrm{~s}, 17-\mathrm{OCH}_{3}\right), 3.41$ (dd, 2'-H), 3.45 (br d, 9-H), 3.57 (dd, 4-H), 3.60 (s, 4-OCH $), 3.90$ (br d, 5-H), 4.48 (d, 1'-H), 4.50 (dd, 17-H), 4.53 (dq, 5"-H), 4.59 (d, 4"-H), 4.83 (m, 14-H), 4.86 (d, 1"-H), 5.22 (br dd, 3-H), 7.17 (m, $\mathrm{C}_{6} \mathrm{H}_{5}$ ), $7.28\left(\mathrm{~m}, \mathrm{C}_{6} \mathrm{H}_{5}\right)$.

(-)-(3R,4S,5S,6R,8R,9R,14R)-5-[4-O-(3-O-Acetyl-2,6- 
dideoxy-3-C-methyl-4- $O$-propionyl- $\alpha$-L-ribo-hexopyranosyl)-3,6-dideoxy-3-dimethylamino- $\beta$-D-glucopyranosyloxy]-6-formylmethyl-9-hydroxy-4-methoxy-8-methyl-11(5-phenylpentyl)-3-propionyloxy-11-aza-pentadecan-14olide (32d)

Reaction of 30d with aqueous difluoroacetic acid gave 32d in $85 \%$ yield by a similar procedure to 11 .

32d: $[\alpha]_{\mathrm{D}}^{25}-79^{\circ}\left(c 0.51, \mathrm{CHCl}_{3}\right)$; FAB-MS $m / z 993$ $(\mathrm{M}+\mathrm{H})^{+}$as $\mathrm{C}_{52} \mathrm{H}_{84} \mathrm{~N}_{2} \mathrm{O}_{16} ;{ }^{1} \mathrm{H}-\mathrm{NMR}\left(300 \mathrm{MHz}, \mathrm{CDCl}_{3}\right) \delta$ : $0.98(\mathrm{~d}, 18-\mathrm{H}), 1.10\left(\mathrm{~d}, 6^{\prime \prime}-\mathrm{H}\right), 1.15\left(\mathrm{~d}, 6^{\prime}-\mathrm{H}\right), 1.17$ (t, 3$\left.\mathrm{OCOCH}_{2} \mathrm{CH}_{3}\right), 1.20$ (t, 4"-OCOCH $\left.\mathrm{CH}_{3}\right), 1.27$ (d, 15-H), 1.33 (quint, $\left.\mathrm{Ph}\left(\mathrm{CH}_{2}\right)_{5}\right), 1.42\left(\mathrm{~s}, 3^{\prime \prime}-\mathrm{CH}_{3}\right), 1.45(\mathrm{~m}, 8-\mathrm{H})$, 1.48 (m, 7-H), $1.63\left(\mathrm{~m}, \mathrm{Ph}\left(\mathrm{CH}_{2}\right)_{5}\right), 1.70$ (dd, 2"-Hax), 2.00 (s, 3'--OCOCH$\left.{ }_{3}\right), 2.56\left(\mathrm{~s}, 3^{\prime}-\mathrm{N}\left(\mathrm{CH}_{3}\right)_{2}\right), 2.81(\mathrm{dd}, 2-\mathrm{H}), 2.96$ (dd, 16-H), 3.23 (m, 4'-H), 3.23 (m, 5'-H), 3.23 (d, 2"Heq), 3.35 (dd, 2'-H), 3.60 (s, 4- $\left.\mathrm{OCH}_{3}\right), 3.65$ (dd, 4-H), 3.89 (br d, 5-H), 4.79 (m, 14-H), 4.86 (d, 1"-H), 5.34 (br dd, $3-\mathrm{H}), 7.18\left(\mathrm{~m}, \mathrm{C}_{6} \mathrm{H}_{5}\right), 7.28\left(\mathrm{~m}, \mathrm{C}_{6} \mathrm{H}_{5}\right), 9.66(\mathrm{~s}, 17-\mathrm{H})$.

9,2'-Di- $O$-acetyl-10,11,12,13-tetrahydro-10,11,12,13-tetrahydroxymidecamycin $\mathrm{A}_{1}$ 18-Dimethylacetal (36)

Reaction of 9-O-acetylmidecamycin $\mathrm{A}_{1}$ 18-dimethylacetal (34) [8b] with acetic anhydride gave $\mathbf{3 5}$ quantitatively by a similar procedure to $\mathbf{1 5}$.

Reaction of 9,2'-di-O-acetylmidecamycin $\mathrm{A}_{1} \quad 18$ dimethylacetal (35) with osmium tetraoxide gave 36 in $41 \%$ yield by a similar procedure to $\mathbf{1 3}$.

36: $[\alpha]_{\mathrm{D}}^{21}-74^{\circ}\left(c\right.$ 1.0, $\mathrm{CHCl}_{3}$ ); FAB-MS $m / z 1012$ $(\mathrm{M}+\mathrm{H})^{+}$as $\mathrm{C}_{47} \mathrm{H}_{81} \mathrm{NO}_{21}$; ${ }^{1} \mathrm{H}-\mathrm{NMR}\left(300 \mathrm{MHz}, \mathrm{CDCl}_{3}\right) \delta$ : 0.96 (d, 19-H), $1.12\left(\mathrm{~s}, 3^{\prime \prime}-\mathrm{CH}_{3}\right), 1.13$ (t, 3-OCOCH $\mathrm{CH}_{3}$ ), $1.14\left(\mathrm{~d}, 6^{\prime \prime}-\mathrm{H}\right), 1.18$ (t, 4"-OCOCH $\left.\mathrm{CH}_{3}\right), 1.27(\mathrm{~d}, 16-\mathrm{H})$, 1.27 (d, 6'-H), 1.43 (br dd, 7-H), 1.53 (br dd, 14-H), 1.63 (m, 6-H), 1.69 (br dd, 14-H), 1.72 (m, 17-H), 1.85 (dd, 2"Hax), 2.02 (d, 2"-Heq), 2.05 (s, 9-OCOCH 3 ), 2.17 (s, 2'$\left.\mathrm{OCOCH}_{3}\right), 2.35$ (m, 8-H), $2.42\left(\mathrm{~s}, 3^{\prime}-\mathrm{N}\left(\mathrm{CH}_{3}\right)_{2}\right), 2.49$ (dd, 2$\mathrm{H}), 2.73(\mathrm{dd}, 2-\mathrm{H}), 2.73\left(\mathrm{t}, 3^{\prime}-\mathrm{H}\right), 3.18\left(\mathrm{~s}, 18-\mathrm{OCH}_{3}\right), 3.23$ $\left(\mathrm{s}, 18-\mathrm{OCH}_{3}\right), 3.34\left(\mathrm{t}, 4^{\prime}-\mathrm{H}\right), 3.57\left(\mathrm{~s}, 4-\mathrm{OCH}_{3}\right), 3.62$ (br d, 12-H), 3.86 (br d, 5-H), 3.90 (dd, 10-H), 4.10 (m, 13-H), 4.38 (dq, 5"-H), 4.40 (dd, 18-H), 4.63 (d, 4"-H), 4.74 (d, 1'H), 5.01 (dd, 2'-H), 5.02 (brd, 9-H), 5.07 (m, 15-H), 5.08 (d, 1"-H), 5.35 (br d, 3-H).

(-)-(3R,4S,5S,6R,8R,9R,14R)-9-Acetoxy-5-[2-O-acetyl-4$O$-(2,6-dideoxy-3-C-methyl-4- $O$-propionyl- $\alpha$-L-ribo-hexopyranosyl)-3,6-dideoxy-3-dimethylamino- $\beta$-D-glucopyranosyloxy]-6-(2,2-dimethoxyethyl)-4-methoxy-8-methyl-11(4-phenylbutyl)-3-propionyloxy-11-aza-pentadecan-14olide (38)

Reaction of $36(714 \mathrm{mg})$ with lead tetraacetate gave crude $37(680 \mathrm{mg})$ by a similar procedure to $\mathbf{1 2}$.

Reaction of crude $37(680 \mathrm{mg})$ with 4-phenylbutylamine gave 38 (63 mg, 8.4\% via two steps based on 36) by a similar procedure to $\mathbf{1 6}$.

38: $[\alpha]_{\mathrm{D}}^{21}-61^{\circ}\left(c 1.0, \mathrm{CHCl}_{3}\right)$; FAB-MS $m / z 1067$ $(\mathrm{M}+\mathrm{H})^{+}$as $\mathrm{C}_{55} \mathrm{H}_{90} \mathrm{~N}_{2} \mathrm{O}_{18} ;{ }^{1} \mathrm{H}-\mathrm{NMR}\left(300 \mathrm{MHz}, \mathrm{CDCl}_{3}\right) \delta$ : $0.92(\mathrm{~d}, 18-\mathrm{H}), 1.13\left(\mathrm{~s}, 3^{\prime \prime}-\mathrm{CH}_{3}\right), 1.14\left(\mathrm{~d}, 6^{\prime \prime}-\mathrm{H}\right), 1.14$ (t, 3$\left.\mathrm{OCOCH}_{2} \mathrm{CH}_{3}\right), 1.18$ (t, 4"-O COCH$\left.{ }_{2} \mathrm{CH}_{3}\right), 1.25$ (d, 15-H), 1.28 (d, 6'-H), 1.40 (br dd, 7-H), 1.44 (quint, $\left.\mathrm{Ph}\left(\mathrm{CH}_{2}\right)_{4}\right)$, $1.61\left(\mathrm{~m}, \mathrm{Ph}\left(\mathrm{CH}_{2}\right)_{4}\right), 1.85$ (dd, 2"-Hax), 1.99 (s, 9$\left.\mathrm{OCOCH}_{3}\right), 2.02$ (d, 2"-Heq), $2.06\left(\mathrm{~s}, 2^{\prime}-\mathrm{OCOCH}_{3}\right), 2.42$ (s, $\left.3^{\prime}-\mathrm{N}\left(\mathrm{CH}_{3}\right)_{2}\right), 2.74\left(\mathrm{t}, 3^{\prime}-\mathrm{H}\right), 2.83(\mathrm{dd}, 2-\mathrm{H}), 3.14(\mathrm{~s}, 17-$ $\left.\mathrm{OCH}_{3}\right), 3.26\left(\mathrm{~s}, 17-\mathrm{OCH}_{3}\right), 3.34\left(\mathrm{~m}, 4^{\prime}-\mathrm{H}\right), 3.36\left(\mathrm{~m}, 5^{\prime}-\mathrm{H}\right)$, 3.47 (br d, 4-H), 3.55 (s, 4- $\left.-\mathrm{OCH}_{3}\right), 3.91$ (br d, 5-H), 4.39 (dq, 5"-H), 4.54 (dd, 17-H), 4.63 (d, 4"-H), 4.72 (d, 1'-H), 4.77 (m, 9-H), 4.92 (br q, 14-H), 5.03 (dd, 2'-H), 5.08 (d, $\left.1^{\prime \prime}-\mathrm{H}\right), 5.17$ (m, 3-H), $7.17\left(\mathrm{~m}, \mathrm{C}_{6} \mathrm{H}_{5}\right), 7.27\left(\mathrm{~m}, \mathrm{C}_{6} \mathrm{H}_{5}\right)$.

(-)-(3R,4S,5S,6R,8R,9R,14R)-5-[4-O-(2,6-Dideoxy-3-Cmethyl-4- $O$-propionyl- $\alpha$-L-ribo-hexopyranosyl)-3,6dideoxy-3-dimethylamino- $\beta$-D-glucopyranosyloxy]-6-(2,2dimethoxyethyl)-9-hydroxy-4-methoxy-8-methyl-11-(4phenylbutyl)-3-propionyloxy-11-aza-pentadecan-14-olide (39)

Reaction of $\mathbf{3 8}$ with methanol gave $\mathbf{3 9}$ in $88 \%$ yield by a similar procedure to $\mathbf{2 6}$.

39: $[\alpha]_{\mathrm{D}}^{21}-58^{\circ}\left(c \quad 0.56, \mathrm{CHCl}_{3}\right)$; FAB-MS $m / z 983$ $(\mathrm{M}+\mathrm{H})^{+}$as $\mathrm{C}_{51} \mathrm{H}_{86} \mathrm{~N}_{2} \mathrm{O}_{16} ;{ }^{1} \mathrm{H}-\mathrm{NMR}\left(300 \mathrm{MHz}, \mathrm{CDCl}_{3}\right) \delta$ : 0.97 (d, 18-H), 1.06 (br dd, 7-H), 1.13 (t, 3- $\mathrm{OCOCH}_{2} \mathrm{CH}_{3}$ ), $1.13\left(\mathrm{~s}, 3^{\prime \prime}-\mathrm{CH}_{3}\right), 1.14$ (d, 6"-H), 1.19 (t, 4"-OCOCH $\mathrm{CH}_{3}$ ), $1.26(\mathrm{~d}, 15-\mathrm{H}), 1.28\left(\mathrm{~d}, 6^{\prime}-\mathrm{H}\right), 1.44$ (br dd, 7-H), 1.48 (m, $\left.\mathrm{Ph}\left(\mathrm{CH}_{2}\right)_{4}\right), 1.64\left(\mathrm{~m}, \mathrm{Ph}\left(\mathrm{CH}_{2}\right)_{4}\right), 1.84$ (dd, 2"-Hax), 2.02 (d, 2"-Heq), 2.51 (s, 3'-N( $\left.\left(\mathrm{CH}_{3}\right)_{2}\right), 2.60$ (dd, 2-H), 2.63 (t, $\left.\mathrm{Ph}\left(\mathrm{CH}_{2}\right)_{4}\right), 2.80$ (dd, 2-H), $3.16\left(\mathrm{~s}, 17-\mathrm{OCH}_{3}\right), 3.28$ (s, 17$\left.\mathrm{OCH}_{3}\right), 3.54$ (br d, 4-H), 3.56 (dd, 2'-H), $3.58\left(\mathrm{~s}, 4-\mathrm{OCH}_{3}\right)$, 3.93 (br d, 5-H), 4.45 (d, 1'-H), 4.47 (dq, 5"-H), 4.63 (d, 4"H), 4.85 (m, 14-H), 5.09 (d, 1"-H), 5.24 (br dd, 3-H), 7.19 $\left(\mathrm{m}, \mathrm{C}_{6} \mathrm{H}_{5}\right), 7.28\left(\mathrm{~m}, \mathrm{C}_{6} \mathrm{H}_{5}\right)$.

(-)-(3R,4S,5S,6R,8R,9R,14R)-5-[4-O-(2,6-Dideoxy-3-Cmethyl-4- $O$-propionyl- $\alpha$-L-ribo-hexopyranosyl)-3,6dideoxy-3-dimethylamino- $\beta$-D-glucopyranosyloxy]-6formylmethyl-9-hydroxy-4-methoxy-8-methyl-11-(4phenylbutyl)-3-propionyloxy-11-aza-pentadecan-14-olide (40) and (-)-(3R,4S,5S,6R,8R,9R,14R)-5-(3,6-Dideoxy-3dimethylamino- $\beta$-D-glucopyranosyloxy)-6-formylmethyl-9hydroxy-4-methoxy-8-methyl-11-(4-phenylbutyl)-3propionyloxy-11-aza-pentadecan-14-olide (41)

Reaction of $\mathbf{3 9}$ with aqueous difluoroacetic acid gave $\mathbf{4 0}$ in $37 \%$ yield and $\mathbf{4 1}$ in $43 \%$ yield by a similar procedure to 11.

40: $[\alpha]_{\mathrm{D}}^{21}-68^{\circ}\left(c \quad 0.40, \mathrm{CHCl}_{3}\right)$; FAB-MS $m / z 937$ $(\mathrm{M}+\mathrm{H})^{+}{ }^{2} \mathrm{C}_{49} \mathrm{H}_{80} \mathrm{~N}_{2} \mathrm{O}_{15} ;{ }^{1} \mathrm{H}-\mathrm{NMR}\left(300 \mathrm{MHz}, \mathrm{CDCl}_{3}\right) \delta$ : 
1.00 (d, 18-H), 1.13 (s, 3"- $\left.\mathrm{CH}_{3}\right), 1.16$ (d, 6"-H), 1.16 (t, 3$\left.\mathrm{OCOCH}_{2} \mathrm{CH}_{3}\right), 1.19$ (t, 4"-OCOCH $\left.\mathrm{CH}_{3}\right), 1.21$ (d, 6'-H), $1.26(\mathrm{~d}, 15-\mathrm{H}), 1.48\left(\mathrm{~m}, \mathrm{Ph}\left(\mathrm{CH}_{2}\right)_{4}\right), 1.64$ (quint, $\left.\mathrm{Ph}\left(\mathrm{CH}_{2}\right)_{4}\right)$, 1.79 (m, 13-H), 1.84 (dd, 2"-Hax), 2.02 (d, 2"-Heq), 2.05 (m, 6-H), $2.38(\mathrm{brd}, 16-\mathrm{H}), 2.52\left(\mathrm{~s}, 3^{\prime}-\mathrm{N}\left(\mathrm{CH}_{3}\right)_{2}\right), 2.64$ (t, $\left.\mathrm{Ph}\left(\mathrm{CH}_{2}\right)_{4}\right), 2.79$ (dd, 2-H), 2.94 (dd, 16-H), 3.28 (m, 4'-H), $3.30\left(\mathrm{~m}, 5^{\prime}-\mathrm{H}\right), 3.54$ (dd, 2'-H), 3.57 (s, 4- $\left.-\mathrm{OCH}_{3}\right), 3.64$ (dd, 4-H), 3.90 (br d, 5-H), 4.42 (d, 1'-H), 4.48 (dq, 5'-H), 4.63 (d, 4"-H), 4.80 (m, 14-H), 5.08 (d, 1"-H), 5.36 (br dd, 3-H), $7.18\left(\mathrm{~m}, \mathrm{C}_{6} \mathrm{H}_{5}\right), 7.28\left(\mathrm{~m}, \mathrm{C}_{6} \mathrm{H}_{5}\right), 9.66(\mathrm{~s}, 17-\mathrm{H})$.

41: $[\alpha]_{\mathrm{D}}^{23}-35^{\circ}\left(c \quad 0.44, \mathrm{CHCl}_{3}\right)$; FAB-MS $m / z 737$ $(\mathrm{M}+\mathrm{H})^{+}$as $\mathrm{C}_{39} \mathrm{H}_{64} \mathrm{~N}_{2} \mathrm{O}_{11} ;{ }^{1} \mathrm{H}-\mathrm{NMR}\left(300 \mathrm{MHz}, \mathrm{CDCl}_{3}\right) \delta$ : 1.00 (d, 18-H), 1.06 (brt, 7-H), 1.17 (t, 3-OCOCH${ }_{2} \mathrm{CH}_{3}$ ), 1.25 (d, 6'-H), 1.27 (d, 15-H), $1.49\left(\mathrm{~m}, \mathrm{Ph}\left(\mathrm{CH}_{2}\right)_{4}\right), 1.64$ (quint, $\left.\mathrm{Ph}\left(\mathrm{CH}_{2}\right)_{4}\right), 1.68(\mathrm{~m}, 13-\mathrm{H}), 1.79(\mathrm{~m}, 13-\mathrm{H}), 2.05$ (brt, 6-H), $2.35\left(\mathrm{t}, 3^{\prime}-\mathrm{H}\right), 2.51\left(\mathrm{~s}, 3^{\prime}-\mathrm{N}\left(\mathrm{CH}_{3}\right)_{2}\right), 2.63$ (t, $\left.\mathrm{Ph}\left(\mathrm{CH}_{2}\right)_{4}\right), 2.80$ (dd, 2-H), 2.99 (dd, 16-H), 3.04 (t, 4'-H), 3.30 (dq, $\left.5^{\prime}-\mathrm{H}\right), 3.52$ (dd, 2'-H), $3.58\left(\mathrm{~s}, 4-\mathrm{OCH}_{3}\right), 3.65$ (dd, 4-H), 3.92 (br d, 5-H), 4.44 (d, 1'-H), 4.81 (m, 14-H), 5.37 (brdd, 3-H), $7.19\left(\mathrm{~m}, \mathrm{C}_{6} \mathrm{H}_{5}\right), 7.29\left(\mathrm{~m}, \mathrm{C}_{6} \mathrm{H}_{5}\right), 9.68$ (s, 17$\mathrm{H})$.

2'-O-Acetyl-9-O-tert-butyldimethylsilyl-10,11,12,13-tetrahydro-10,11,12,13-tetrahydroxyrokitamycin 18-dimethylacetal (45)

To a mixture of 2'-O-Acetyl-9-O-tert-butyldimethylsilylrokitamycin 18-dimethylacetal (44) [22b, 32] (1.08 g) in acetone $(27 \mathrm{ml})$ and water $(4.2 \mathrm{ml})$ were added $N$ methylmorpholine- $N$-oxide $(0.49 \mathrm{ml})$ and $4 \%$ aqueous osmium tetraoxide $(1.0 \mathrm{ml})$, and the mixture was stirred at room temperature for 24 hours. After the reaction mixture was concentrated under reduced pressure, the concentrate was extracted with ethyl acetate $(40 \mathrm{ml})$, and then the organic layer was washed with saturated brine $(30 \mathrm{ml})$. The organic layer was dried over anhydrous sodium sulfate and filtered. Then, the filtrate was concentrated under reduced pressure, and the resulting residue was purified by flash silica gel column chromatography (chloroform/methanol $(40: 1 \sim 30: 1))$ to obtain $45(394 \mathrm{mg}, 34 \%)$ as a colorless solid.

45: $[\alpha]_{\mathrm{D}}^{22}-81^{\circ}\left(c\right.$ 1.0, $\mathrm{CHCl}_{3}$ ); FAB-MS $m / z 1098$ $(\mathrm{M}+\mathrm{H})^{+}$as $\mathrm{C}_{52} \mathrm{H}_{95} \mathrm{NO}_{21} \mathrm{Si}$; ${ }^{1} \mathrm{H}-\mathrm{NMR}\left(300 \mathrm{MHz}, \mathrm{CDCl}_{3}\right.$ ) $\delta$ : $0.04\left(\mathrm{~s}, 9-\mathrm{OSi}\left(\mathrm{CH}_{3}\right){ }_{2}{ }^{t} \mathrm{Bu}\right), 0.07\left(\mathrm{~s}, 9-\mathrm{OSi}\left(\mathrm{CH}_{3}\right)_{2}{ }^{t} \mathrm{Bu}\right)$, $0.93(\mathrm{~d}, 19-\mathrm{H}), 0.93\left(\mathrm{~s}, 9-\mathrm{OSi}\left(\mathrm{CH}_{3}\right)_{2}{ }^{t} \mathrm{Bu}\right), 0.98$ (t, 4"$\left.\mathrm{OCO}\left(\mathrm{CH}_{2}\right)_{2} \mathrm{CH}_{3}\right), 1.07$ (d, 6"-H), $1.16\left(\mathrm{t}, 3^{\prime \prime}-\mathrm{OCOCH}_{2} \mathrm{CH}_{3}\right)$, 1.22 (d, 6'-H), 1.29 (d, 16-H), 1.42 (s, 3"- $\left.\mathrm{CH}_{3}\right), 1.55$ (br dd, 14-H), 1.69 (dd, 2"-Hax), 1.70 (sext, 4"-OCO $\left(\mathrm{CH}_{2}\right)_{2} \mathrm{CH}_{3}$ ), $2.02\left(\mathrm{~s}, \quad 2^{\prime}-\mathrm{OCOCH}_{3}\right), \quad 2.29,2.32$ (each q, 3"$\left.\mathrm{OCOCH}_{2} \mathrm{CH}_{3}\right), 2.38$ (t, 4"-OCO $\left.\left(\mathrm{CH}_{2}\right)_{2} \mathrm{CH}_{3}\right), 2.43$ (s, 3'$\left.\mathrm{N}\left(\mathrm{CH}_{3}\right)_{2}\right), 2.59$ (t, 3'-H), 3.15 (t, 4'-H), 3.21 (d, 2"'-Heq), $3.31\left(\mathrm{~s}, 18-\mathrm{OCH}_{3}\right), 3.35\left(\mathrm{~s}, 18-\mathrm{OCH}_{3}\right), 3.48\left(\mathrm{~s}, 4-\mathrm{OCH}_{3}\right)$,
3.63 (m, 12-H), 3.89 (br d, 5-H), 4.49 (dq, 5"-H), 4.58 (d, 4"-H), 4.82 (d, 1"-H), 4.97 (dd, 2'-H).

(-)-(3R,4S,5S,6R,8R,9R,14R)-5-[2-O-Acetyl-4-O-(4-Obutyryl-2,6-dideoxy-3-C-methyl-3- $O$-propionyl- $\alpha$-L-ribohexopyranosyl)-3,6-dideoxy-3-dimethylamino- $\beta$-D-glucopyranosyloxy]-9-O-tert-butyldimethylsilyl-6-(2,2dimethoxyethyl)-3-hydroxy-4-methoxy-8-methyl-11-(4phenylbutyl)-11-aza-pentadecan-14-olide (47)

Reaction of $\mathbf{4 5}(389 \mathrm{mg})$ with lead tetraacetate gave crude $46(370 \mathrm{mg})$ by a similar procedure to $\mathbf{1 2}$.

Reaction of crude $46(370 \mathrm{mg})$ with 4-phenylbutylamine gave 47 (40.0 mg, 9.8\% via two steps based on 45 ) by a similar procedure to $\mathbf{1 6}$.

47: $[\alpha]_{\mathrm{D}}^{21}-76^{\circ}\left(c\right.$ 1.0, $\left.\mathrm{CHCl}_{3}\right)$; FAB-MS $m / z 1153$ $(\mathrm{M}+\mathrm{H})^{+}$as $\mathrm{C}_{60} \mathrm{H}_{104} \mathrm{~N}_{2} \mathrm{O}_{17} \mathrm{Si}$; ${ }^{1} \mathrm{H}-\mathrm{NMR}\left(300 \mathrm{MHz}, \mathrm{CDCl}_{3}\right)$ $\delta$ : 0.03 (s, 9-OSi $\left.\left(\mathrm{CH}_{3}\right)_{2}{ }^{t} \mathrm{Bu}\right), 0.87$ (s, 9-OSi $\left.\left(\mathrm{CH}_{3}\right)_{2}{ }^{t} \mathrm{Bu}\right), 0.90$ (d, 18-H), 0.98 (t, 4"-OCO $\left.\left(\mathrm{CH}_{2}\right)_{2} \mathrm{CH}_{3}\right), 1.07$ (d, 6"-H), 1.15 (t, 3"-OCOCH$\left.{ }_{2} \mathrm{CH}_{3}\right), 1.20\left(\mathrm{~d}, 6^{\prime}-\mathrm{H}\right), 1.22(\mathrm{~d}, 15-\mathrm{H}), 1.42$ (s, 3"- $\left.\mathrm{CH}_{3}\right), 1.55$ (m, $\left.\mathrm{Ph}\left(\mathrm{CH}_{2}\right)_{4}\right), 1.67$ (dd, 2"-Hax), 1.70 (sext, 4"-OCO $\left.\left(\mathrm{CH}_{2}\right)_{2} \mathrm{CH}_{3}\right), 2.04$ (s, 2'-OCOCH$\left.{ }_{3}\right), 2.29,2.32$ (each q, 3"- $\mathrm{OCOCH}_{2} \mathrm{CH}_{3}$ ), 2.38 (t, 4"-OCO $\left(\mathrm{CH}_{2}\right)_{2} \mathrm{CH}_{3}$ ), $2.42\left(\mathrm{~s}, 3^{\prime}-\mathrm{N}\left(\mathrm{CH}_{3}\right)_{2}\right), 3.01(\mathrm{brd}, 4-\mathrm{H}), 3.14\left(\mathrm{t}, 4^{\prime}-\mathrm{H}\right), 3.20$ (d, 2"-Heq), $3.32\left(\mathrm{~s}, 17-\mathrm{OCH}_{3}\right), 3.49\left(\mathrm{~s}, 4-\mathrm{OCH}_{3}\right), 3.96$ (br d, 5-H), 4.48 (dq, 5"-H), 4.49 (d, 1'-H), 4.57 (d, 4"-H), $4.81\left(\mathrm{~d}, 1^{\prime \prime}-\mathrm{H}\right), 4.97$ (dd, 2'-H), $5.12(\mathrm{~m}, 14-\mathrm{H}), 7.17$ (m, $\mathrm{Ph}), 7.28(\mathrm{~m}, \mathrm{Ph})$.

(-)-(3R,4S,5S,6R,8R,9R,14R)-9-O-tert-Butyldimethylsilyl5-[4-O-(4-O-butyryl-2,6-dideoxy-3-C-methyl-3- $O$-propionyl- $\alpha$-L-ribo-hexopyranosyl)-3,6-dideoxy-3-dimethylamino- $\beta$-D-glucopyranosyloxy]-6-(2,2-dimethoxyethyl)-3hydroxy-4-methoxy-8-methyl-11-(4-phenylbutyl)-11-azapentadecan-14-olide (48)

Reaction of $\mathbf{4 7}$ with methanol gave $\mathbf{4 8}$ in $74 \%$ yield by a similar procedure to $\mathbf{2 6}$.

48: $[\alpha]_{\mathrm{D}}^{22}-70^{\circ}\left(c \quad 0.85, \mathrm{CHCl}_{3}\right)$; FAB-MS $m / z 1111$ $(\mathrm{M}+\mathrm{H})^{+}$as $\mathrm{C}_{58} \mathrm{H}_{102} \mathrm{~N}_{2} \mathrm{O}_{16} \mathrm{Si} ;{ }^{1} \mathrm{H}-\mathrm{NMR}\left(300 \mathrm{MHz}, \mathrm{CDCl}_{3}\right)$ $\delta$ : 0.06 (s, 9-OSi $\left.\left(\mathrm{CH}_{3}\right)_{2}{ }^{t} \mathrm{Bu}\right), 0.89$ (s, 9-OSi $\left.\left(\mathrm{CH}_{3}\right)_{2}{ }^{t} \mathrm{Bu}\right), 0.90$ (d, 18-H), 0.99 (t, 4"-OCO $\left.\left(\mathrm{CH}_{2}\right)_{2} \mathrm{CH}_{3}\right), 1.09$ (d, 6"-H), 1.14 (t, 3"-OCOCH$\left.{ }_{2} \mathrm{CH}_{3}\right), 1.22(\mathrm{~d}, 15-\mathrm{H}), 1.22\left(\mathrm{~d}, 6^{\prime}-\mathrm{H}\right), 1.42(\mathrm{~s}$, 3"- $\left.\mathrm{CH}_{3}\right), 1.57$ (m, Ph $\left.\left(\mathrm{CH}_{2}\right)_{4}\right), 1.70$ (dd, 2"-Hax), 1.70 (sext, 4"-OCO $\left(\mathrm{CH}_{2}\right)_{2} \mathrm{CH}_{3}$ ), 2.30, 2.32 (each q, 3"-OCOCH $\mathrm{CH}_{3}$ ), 2.39 (t, 4"-OCO $\left.\left(\mathrm{CH}_{2}\right)_{2} \mathrm{CH}_{3}\right), 2.53\left(\mathrm{~s}, 3^{\prime}-\mathrm{N}\left(\mathrm{CH}_{3}\right)_{2}\right), 2.61(\mathrm{t}$, $\left.\mathrm{Ph}\left(\mathrm{CH}_{2}\right)_{4}\right), 3.10$ (m, 4-H), 3.12 (d, 2"-Heq), 3.32 (s, 17$\left.\mathrm{OCH}_{3}\right), 3.33\left(\mathrm{~s}, 17-\mathrm{OCH}_{3}\right), 3.49\left(\mathrm{dd}, 2^{\prime}-\mathrm{H}\right), 3.61$ (s, 4$\left.\mathrm{OCH}_{3}\right), 3.92(\mathrm{~m}, 5-\mathrm{H}), 4.34\left(\mathrm{~d}, 1^{\prime}-\mathrm{H}\right), 4.47$ (t, 17-H), 4.55 (m, 5"-H), 4.59 (d, 4"-H), 4.83 (d, 1"-H), 5.19 (m, 14-H), $7.17(\mathrm{~m}, \mathrm{Ph}), 7.28$ (m, Ph).

(-)-(3R,4S,5S,6R,8R,9R,14R)-9-O-tert-Butyldimethylsilyl- 
5-[4- $O$-(4- $O$-butyryl-2,6-dideoxy-3- $C$-methyl-3- $O$-propionyl- $\alpha$-L-ribo-hexopyranosyl)-3,6-dideoxy-3-dimethylamino- $\beta$-D-glucopyranosyloxy]-6-formylmethyl-3-hydroxy-4-methoxy-8-methyl-11-(4-phenylbutyl)-11-azapentadecan-14-olide (49)

Reaction of 48 with aqueous difluoroacetic acid gave $\mathbf{4 9}$ in $98 \%$ yield by a similar procedure to 11 .

49: $[\alpha]_{\mathrm{D}}^{20}-61^{\circ}\left(c 0.60, \mathrm{CHCl}_{3}\right)$; FAB-MS $m / z 1065$ $(\mathrm{M}+\mathrm{H})^{+}$as $\mathrm{C}_{56} \mathrm{H}_{96} \mathrm{~N}_{2} \mathrm{O}_{15} \mathrm{Si}$; ${ }^{1} \mathrm{H}-\mathrm{NMR}\left(300 \mathrm{MHz}, \mathrm{CDCl}_{3}\right.$ ) $\delta$ : 0.02 (s, 9-OSi $\left.\left(\mathrm{CH}_{3}\right)_{2}{ }^{t} \mathrm{Bu}\right), 0.03$ (s, 9-OSi $\left.\left(\mathrm{CH}_{3}\right)_{2}{ }^{t} \mathrm{Bu}\right)$, 0.87 (s, 9-OSi $\left.\left(\mathrm{CH}_{3}\right)_{2}{ }^{t} \mathrm{Bu}\right), 0.93(\mathrm{~d}, 18-\mathrm{H}), 0.99$ (t, 4"$\left.\mathrm{OCO}\left(\mathrm{CH}_{2}\right)_{2} \mathrm{CH}_{3}\right), 1.09$ (d, 6"-H), 1.14 (t, 3"-OCOCH${ }_{2} \mathrm{CH}_{3}$ ), $1.19\left(\mathrm{~d}, 6^{\prime}-\mathrm{H}\right), 1.22(\mathrm{~d}, 15-\mathrm{H}), 1.42\left(\mathrm{~s}, 3^{\prime \prime}-\mathrm{CH}_{3}\right), 1.57$ (m, $\left.\mathrm{Ph}\left(\mathrm{CH}_{2}\right)_{4}\right), 1.65$ (dd, 2"-Hax), 1.70 (sext, 4"$\left.\mathrm{OCO}\left(\mathrm{CH}_{2}\right)_{2} \mathrm{CH}_{3}\right), 2.39$ (t, 4"-OCO $\left.\left(\mathrm{CH}_{2}\right)_{2} \mathrm{CH}_{3}\right), 2.53$ (s, 3'$\left.\mathrm{N}\left(\mathrm{CH}_{3}\right)_{2}\right), 2.62$ (t, $\left.\mathrm{Ph}\left(\mathrm{CH}_{2}\right)_{4}\right), 2.74(\mathrm{dd}, 2-\mathrm{H}), 2.97$ (dd, 16H), 3.23 (d, 2"-Heq), 3.43 (dd, 2'-H), 3.59 (s, 4- $\left.\mathrm{OCH}_{3}\right)$, 3.81 (br d, 5-H), 4.17 (m, 3-H), 4.34 (d, 1'-H), 4.53 (dq, 5"H), 4.59 (d, 4"-H), 4.84 (d, 1"-H), 5.14 (m, 14-H), 7.18 (m, $\mathrm{Ph}), 7.28(\mathrm{~m}, \mathrm{Ph}), 9.70(\mathrm{~s}, 17-\mathrm{H})$.

(-)-(3R,4S,5S,6R,8R,9R,14R)-5-[4-O-(4-O-Butyryl-2,6dideoxy-3-C-methyl-3- $O$-propionyl- $\alpha$-L-ribo-hexopyranosyl)-3,6-dideoxy-3-dimethylamino- $\beta$-D-glucopyranosyloxy]-6-formylmethyl-3,9-dihydroxy-4-methoxy-8-methyl11-(4-phenylbutyl)-11-aza-pentadecan-14-olide (50)

To a solution of $49(13.0 \mathrm{mg})$ in tetrahydrofuran $(0.5 \mathrm{ml})$ and acetic acid $(0.5 \mathrm{ml})$ was added $1 \mathrm{M}$ tetrabutylammonium fluoride tetrahydrofuran solution $(60 \mu \mathrm{l})$, and the mixture was stirred at $60^{\circ} \mathrm{C}$ for 48 hours. The reaction mixture was slowly poured into saturated aqueous sodium hydrogencarbonate solution $(10 \mathrm{ml})$, and extracted with ethyl acetate $(25 \mathrm{ml})$. The organic layer was washed successively with water $(15 \mathrm{ml})$, saturated aqueous sodium hydrogencarbonate solution $(15 \mathrm{ml})$ and saturated brine $(15 \mathrm{ml})$, dried over anhydrous sodium sulfate, and then filtered. The filtrate was concentrated under reduced pressure, and the resulting residue was purified by preparative TLC (chloroform/methanol/aqueous ammonia $(20: 1: 0.1))$ to obtain $\mathbf{5 0}(6.3 \mathrm{mg}, 54 \%)$.

50: $[\alpha]_{\mathrm{D}}^{22}-95^{\circ}\left(c \quad 0.38, \mathrm{CHCl}_{3}\right)$; FAB-MS $m / z 951$ $(\mathrm{M}+\mathrm{H})^{+}$as $\mathrm{C}_{50} \mathrm{H}_{82} \mathrm{~N}_{2} \mathrm{O}_{15} ;{ }^{1} \mathrm{H}-\mathrm{NMR}\left(300 \mathrm{MHz}, \mathrm{CDCl}_{3}\right.$ ) $\delta: 0.91(\mathrm{~d}, 18-\mathrm{H}), 0.98\left(\mathrm{t}, 4^{\prime \prime}-\mathrm{OCO}\left(\mathrm{CH}_{2}\right)_{2} \mathrm{CH}_{3}\right), 1.09\left(\mathrm{~d}, 6^{\prime \prime}-\right.$ $\mathrm{H}), 1.13\left(\mathrm{t}, 3^{\prime \prime}-\mathrm{OCOCH}_{2} \mathrm{CH}_{3}\right), 1.17$ (d, 6'-H), 1.26 (d, 15$\mathrm{H}), 1.42\left(\mathrm{~s}, 3^{\prime \prime}-\mathrm{CH}_{3}\right), 1.46\left(\mathrm{~m}, \mathrm{Ph}\left(\mathrm{CH}_{2}\right)_{4}\right), 1.63(\mathrm{~m}$, $\left.\mathrm{Ph}\left(\mathrm{CH}_{2}\right)_{4}\right), 1.69$ (sext, 4"-OCO $\left.\left(\mathrm{CH}_{2}\right)_{2} \mathrm{CH}_{3}\right), 2.38$ (t, 4"$\left.\mathrm{OCO}\left(\mathrm{CH}_{2}\right)_{2} \mathrm{CH}_{3}\right), 2.54\left(\mathrm{~s}, 3^{\prime}-\mathrm{N}\left(\mathrm{CH}_{3}\right)_{2}\right), 2.62\left(\mathrm{t}, \mathrm{Ph}\left(\mathrm{CH}_{2}\right)_{4}\right)$, 2.80 (dd, 2-H), 3.02 (dd, 16-H), 3.19 (m, 4' $\left.{ }^{\prime}-\mathrm{H}\right), 3.19$ (m, $5^{\prime}-$ $\mathrm{H}), 3.23$ (d, 2"-Heq), 3.35 (dd, 2'-H), 3.59 (s, 4- $\mathrm{OCH}_{3}$ ), 3.82 (br d, 5-H), 4.28 (br dd, 3-H), 4.34 (d, 1'-H), 4.52 (dq, 5"-H), 4.59 (d, 4"-H), 4.85 (d, 1"-H), 4.94 (m, 14-H), 7.18 (m, Ph), $7.28(\mathrm{~m}, \mathrm{Ph}), 9.75(\mathrm{~s}, 17-\mathrm{H})$.

(-)-(3R,4S,5S,6R,8R,9R,14R)-5-[4-O-(3-O-Acetyl-2,6dideoxy-3-C-methyl-4- $O$-propionyl- $\alpha$-L-ribo-hexopyranosyl)-3,6-dideoxy-3-dimethylamino- $\beta$-D-glucopyranosyloxy]-6-formylmethyl-9-hydroxy-4-methoxy-8-methyl-3propionyloxy-11-(3-(pyridin-4-yl)propyl)-11-aza-pentadecan-14-olide (51)

To a solution of $22(30.0 \mathrm{mg})$ in methanol $(0.60 \mathrm{ml})$ were added acetic acid $(9.6 \mu 1)$ and 3-(pyridin-4-yl)propanal $(6.3 \mu \mathrm{l})$ under ice cooling, and the mixture was stirred for 30 minutes. To the resulting solution was added sodium cyanoborohydride $(6.3 \mathrm{mg})$, and the mixture was stirred for 12 hours with gradually warming to room temperature. Ethyl acetate $(3.0 \mathrm{ml})$ and saturated aqueous sodium hydrogencarbonate solutiom $(3.0 \mathrm{ml})$ were added, and the mixture was stirred at room temperature for 30 minutes. The organic layer was separated, and then the aqueous layer was twice extracted with ethyl acetate $(5.0 \mathrm{ml})$. The organic layers were combined and washed successively with saturated aqueous sodium hydrogencarbonate solution $(10 \mathrm{ml})$ and saturated brine $(10 \mathrm{ml})$, dried over anhydrous sodium sulfate, and then filtered. The filtrate was concentrated under reduced pressure, and the resulting residue was purified by preparative TLC (chloroform/ methanol/aqueous ammonia $(20: 1: 0.1))$ to obtain 18 dimethylacetal of $\mathbf{5 1}$ (30.0 mg, 88\%).

18-Dimethylacetal of 51: $[\alpha]_{\mathrm{D}}^{22}-70^{\circ}\left(c 0.50, \mathrm{CHCl}_{3}\right)$; FAB-MS $m / z 1012(\mathrm{M}+\mathrm{H})^{+}$as $\mathrm{C}_{51} \mathrm{H}_{85} \mathrm{~N}_{3} \mathrm{O}_{17} ;{ }^{1} \mathrm{H}-\mathrm{NMR}$ $\left(300 \mathrm{MHz}, \mathrm{CDCl}_{3}\right) \delta: 0.94(\mathrm{~d}, 18-\mathrm{H}), 1.07\left(\mathrm{~d}, 6^{\prime \prime}-\mathrm{H}\right), 1.11(\mathrm{t}$, $3-\mathrm{OCOCH}_{2} \mathrm{CH}_{3}$ ), 1.17 (t, 4"-OCOCH $\left.\mathrm{CH}_{3}\right), 1.19$ (d, 6'-H), $1.24(\mathrm{~d}, 15-\mathrm{H}), 1.40\left(\mathrm{~s}, 3^{\prime \prime}-\mathrm{CH}_{3}\right), 1.58(\mathrm{~m}, 8-\mathrm{H}), 1.58(\mathrm{~m}$, 16-H), 1.68 (dd, 2"-Hax), 2.00 (s, 3"-OCOCH ${ }_{3}$ ), 2.53 (s, 3'$\left.\mathrm{N}\left(\mathrm{CH}_{3}\right)_{2}\right), 2.81(\mathrm{dd}, 2-\mathrm{H}), 3.10\left(\mathrm{~s}, 17-\mathrm{OCH}_{3}\right), 3.21$ (d, 2"Heq), 3.23 (m, 4'-H), 3.23 (m, 5'-H), 3.24 (s, 17- $\mathrm{OCH}_{3}$ ), 3.38 (dd, 2'-H), 3.56 (br d, 4-H), 3.58 (s, 4- $\left.-\mathrm{OCH}_{3}\right), 3.88$ (br d, 5-H), 4.47 (d, 1'-H), 4.49 (dq, 5"'-H), 4.57 (d, 4"-H), 4.81 (ddq, 14-H), 4.84 (d, 1"-H), 5.19 (br dd, 3-H), 7.10 (dd, pyridine), 8.48 (dd, pyridine).

Reaction of 18-dimethylacetal of $\mathbf{5 1}$ with aqueous difluoroacetic acid gave $\mathbf{5 1}$ in $84 \%$ yield by a similar procedure to 11.

51: $[\alpha]_{\mathrm{D}}^{20}-84^{\circ}\left(c \quad 0.50, \mathrm{CHCl}_{3}\right.$ ); FAB-MS $m / z 966$ $(\mathrm{M}+\mathrm{H})^{+}$as $\mathrm{C}_{49} \mathrm{H}_{79} \mathrm{~N}_{3} \mathrm{O}_{16} ;{ }^{1} \mathrm{H}-\mathrm{NMR}\left(300 \mathrm{MHz}, \mathrm{CDCl}_{3}\right) \delta$ : 0.95 (d, 18-H), 1.07 (d, 6"-H), 1.13 (d, 6'-H), 1.14 (t, 3$\left.\mathrm{OCOCH}_{2} \mathrm{CH}_{3}\right), 1.17$ (t, 4"-OCOCH $\left.\mathrm{CH}_{3}\right), 1.24(\mathrm{~d}, 15-\mathrm{H})$, 1.40 (s, 3"- $\mathrm{CH}_{3}$ ), 1.43 (m, 8-H), 1.68 (dd, 2"-Hax), 1.98 (s, $\left.3^{\prime \prime}-\mathrm{OCOCH}_{3}\right), 2.54\left(\mathrm{~s}, 3^{\prime}-\mathrm{N}\left(\mathrm{CH}_{3}\right)_{2}\right), 2.79(\mathrm{dd}, 2-\mathrm{H}), 2.95$ (dd, 16-H), 3.21 (d, 2"'-Heq), 3.21 (m, 4'-H), 3.21 (m, 5' H), $3.31\left(\mathrm{dd}, 2^{\prime}-\mathrm{H}\right), 3.58\left(\mathrm{~s}, 4-\mathrm{OCH}_{3}\right), 3.62(\mathrm{dd}, 4-\mathrm{H}), 3.87$ (br d, 5-H), 4.42 (d, 1'-H), 4.48 (dq, 5"-H), 4.57 (d, 4"-H), 
4.77 (ddq, 14-H), 4.84 (d, 1"-H), 5.31 (br dd, 3-H), 7.10 (dd, pyridine), 8.48 (dd, pyridine), 9.63 (s, 17-H).

$(-)-(3 R, 4 S, 5 S, 6 R, 8 R, 9 R, 14 R)-5-[4-O-(3-O$-Acetyl-2,6dideoxy-3-C-methyl-4- $O$-propionyl- $\alpha$-L-ribo-hexopyranosyl)-3,6-dideoxy-3-dimethylamino- $\beta$-D-glucopyranosyloxy]-6-formylmethyl-9-hydroxy-4-methoxy-8-methyl-3propionyloxy-11-(3-(quinolin-4-yl)propyl)-11-aza-pentadecan-14-olide (52)

Reaction of 12 with 3-(quinolin-4-yl)propylamine gave 9,2'-diacetate 18-dimethylacetal of $\mathbf{5 2}$ in $9.4 \%$ yield by a similar procedure to $\mathbf{1 6}$.

9,2'-Diacetate 18-dimethylacetal of 52: $[\alpha]_{\mathrm{D}}^{26}-79^{\circ}(c$ 1.0, $\mathrm{CHCl}_{3}$ ); FAB-MS m/z $1146(\mathrm{M}+\mathrm{H})^{+}$as $\mathrm{C}_{59} \mathrm{H}_{91} \mathrm{~N}_{3} \mathrm{O}_{19}$; ${ }^{1} \mathrm{H}-\mathrm{NMR}\left(300 \mathrm{MHz}, \mathrm{CDCl}_{3}\right) \delta: 0.93(\mathrm{~d}, 18-\mathrm{H}), 1.06\left(\mathrm{~d}, 66^{\prime \prime}\right.$ $\mathrm{H}), 1.13\left(\mathrm{t}, 3-\mathrm{OCOCH}_{2} \mathrm{CH}_{3}\right), 1.18$ (t, 4"-OCOCH $\mathrm{CH}_{3}$ ), $1.22\left(\mathrm{~d}, 6^{\prime}-\mathrm{H}\right), 1.23(\mathrm{~d}, 15-\mathrm{H}), 1.40\left(\mathrm{~s}, 3^{\prime \prime}-\mathrm{CH}_{3}\right), 1.54(\mathrm{~m}$, 16-H), 1.67 (dd, 2"-Hax), 1.86 (m, quinoline- $\left.\left(\mathrm{CH}_{2}\right)_{3}\right), 1.96$ $\left(\mathrm{s}, 9-\mathrm{OCOCH}_{3}\right), 2.01\left(\mathrm{~s}, 3^{\prime \prime}-\mathrm{OCOCH}_{3}\right), 2.03$ (s, 2'$\left.\mathrm{OCOCH}_{3}\right), 2.43\left(\mathrm{~s}, 3^{\prime}-\mathrm{N}\left(\mathrm{CH}_{3}\right)_{2}\right), 2.84(\mathrm{dd}, 2-\mathrm{H}), 3.07$ (t, quinoline- $\left.\left(\mathrm{CH}_{2}\right)_{3}\right), 3.12$ (s, 17- $\left.\mathrm{OCH}_{3}\right), 3.19$ (d, 2"-Heq), $3.24\left(\mathrm{~s}, 17-\mathrm{OCH}_{3}\right), 3.48$ (dd, 4-H), $3.54\left(\mathrm{~s}, 4-\mathrm{OCH}_{3}\right), 3.89$ (br d, 5-H), 4.47 (dq, 5"'-H), 4.56 (d, 4"-H), 4.67 (d, 1'-H), 4.80 (d, 1"-H), 4.84 (m, 9-H), 4.93 (m, 14-H), 4.97 (dd, 2'-H), 5.17 (m, 3-H), 7.25 (d, quinoline), 7.56 (ddd, quinoline), 7.70 (ddd, quinoline), 8.06 (br d, quinoline), 8.09 (br d, quinoline), 8.79 (d, quinoline).

9,2'-Diacetate 18-dimethylacetal of $\mathbf{5 2}(135 \mathrm{mg})$ was dissolved in methanol $(5.4 \mathrm{ml})$, and the reaction mixture was stirred at $45^{\circ} \mathrm{C}$ for 44 hours, and then concentrated under reduced pressure. The resulting residue was purified by preparative TLC (chloroform/methanol $(10: 1)$ ) to obtain 18-dimethyacetal of $\mathbf{5 2}(58.0 \mathrm{mg}, 46 \%)$.

18-Dimethyacetal of 52: $[\alpha]_{\mathrm{D}}^{26}-64^{\circ}\left(c 1.30, \mathrm{CHCl}_{3}\right)$; FAB-MS $m / z 1062(\mathrm{M}+\mathrm{H})^{+}$as $\mathrm{C}_{55} \mathrm{H}_{87} \mathrm{~N}_{3} \mathrm{O}_{17} ;{ }^{1} \mathrm{H}-\mathrm{NMR}$ $\left(300 \mathrm{MHz}, \mathrm{CDCl}_{3}\right) \delta: 0.96(\mathrm{~d}, 18-\mathrm{H}), 1.09$ (d, 6"-H), 1.13 (t, $\left.3-\mathrm{OCOCH}_{2} \mathrm{CH}_{3}\right), 1.19$ (t, 4"-OCOCH $\left.\mathrm{CH}_{3}\right), 1.21$ (d, 6'-H), 1.25 (d, 15-H), 1.42 (s, 3"- $\left.\mathrm{CH}_{3}\right), 1.69$ (dd, 2"-Hax), 1.91 (m, 16-H), 1.94 (quint, quinoline- $\left.\left(\mathrm{CH}_{2}\right)_{3}\right), 2.01$ (s, 3"$\left.\mathrm{OCOCH}_{3}\right), 2.55\left(\mathrm{~s}, 3^{\prime}-\mathrm{N}\left(\mathrm{CH}_{3}\right)_{2}\right), 2.59(\mathrm{dd}, 2-\mathrm{H}), 2.83(\mathrm{dd}$, 2-H), 3.09 (t, quinoline- $\left.\left(\mathrm{CH}_{2}\right)_{3}\right), 3.13\left(\mathrm{~s}, 17-\mathrm{OCH}_{3}\right), 3.22$ (d, 2"-Heq), 3.23 (m, 4'-H), 3.23 (m, 5'-H), 3.26 (s, 17$\left.\mathrm{OCH}_{3}\right), 3.40$ (dd, 2'-H), 3.59 (dd, 4-H), 3.60 (s, 4- $\left.\mathrm{OCH}_{3}\right)$, 3.90 (br d, 5-H), 4.48 (d, 1'-H), 4.52 (dq, 5"-H), 4.59 (d, 4"H), 4.83 (m, 14-H), 4.86 (d, 1"-H), 5.24 (br dd, 3-H), 7.25 (d, quinoline), 7.58 (ddd, quinoline), 7.71 (ddd, quinoline), 8.03 (brd, quinoline), 8.12 (brd, quinoline), 8.81 (d, quinoline).

Reaction of 18-dimethylacetal of $\mathbf{5 2}$ with aqueous difluoroacetic acid gave $\mathbf{5 2}$ in $86 \%$ yield by a similar procedure to $\mathbf{1 1 .}$
52: $[\alpha]_{\mathrm{D}}^{26}-71^{\circ}\left(c \quad 0.58, \mathrm{CHCl}_{3}\right)$; FAB-MS $m / z 1016$ $(\mathrm{M}+\mathrm{H})^{+}$as $\mathrm{C}_{53} \mathrm{H}_{81} \mathrm{~N}_{3} \mathrm{O}_{16} ;{ }^{1} \mathrm{H}-\mathrm{NMR}\left(300 \mathrm{MHz}, \mathrm{CDCl}_{3}\right) \delta$ : 0.98 (d, 18-H), 1.09 (d, 6"-H), 1.15 (d, 6'-H), 1.16 (t, 3$\left.\mathrm{OCOCH}_{2} \mathrm{CH}_{3}\right), 1.19$ (t, 4"-OCOCH $\left.\mathrm{CH}_{3}\right), 1.25$ (d, 15-H), 1.42 (s, 3"- $\mathrm{CH}_{3}$ ), 1.48 (br s, 8-H), 1.70 (dd, 2"-Hax), 1.94 (m, quinoline- $\left.\left(\mathrm{CH}_{2}\right)_{3}\right), 2.00\left(\mathrm{~s}, 3^{\prime \prime}-\mathrm{OCOCH}_{3}\right), 2.56\left(\mathrm{~s}, 3^{\prime}-\right.$ $\left.\mathrm{N}\left(\mathrm{CH}_{3}\right)_{2}\right), 2.82(\mathrm{dd}, 2-\mathrm{H}), 2.98(\mathrm{dd}, 16-\mathrm{H}), 3.01(\mathrm{dt}$, quinoline- $\left.\left(\mathrm{CH}_{2}\right)_{3}\right), 3.22\left(\mathrm{~m}, 4^{\prime}-\mathrm{H}\right), 3.22\left(\mathrm{~m}, 5^{\prime}-\mathrm{H}\right), 3.22(\mathrm{~d}$, 2"-Heq), 3.34 (dd, 2'-H), 3.60 (s, 4-OCH ${ }_{3}$ ), 3.65 (dd, 4-H), 3.89 (br d, 5-H), 4.45 (d, 1'-H), 4.51 (dq, 5"-H), 4.59 (d, 4"H), 4.79 (m, 14-H), 4.86 (d, 1"-H), 5.34 (br dd, 3-H), 7.25 (d, quinoline), 7.57 (ddd, quinoline), 7.71 (ddd, quinoline), 8.03 (dd, quinoline), 8.12 (dd, quinoline), 8.81 (d, quinoline), 9.65 (s, 17-H).

(-)-(3R,4S,5S,6R,8R,9R,14R)-5-[4-O-(3-O-Acetyl-2,6dideoxy-3-C-methyl-4- $O$-propionyl- $\alpha$-L-ribo-hexopyranosyl)-3,6-dideoxy-3-dimethylamino- $\beta$-D-glucopyranosyloxy]-6-formylmethyl-9-hydroxy-4-methoxy-11-(3-(6methoxyquinolin-4-yl)propyl)-8-methyl-3-propionyloxy11-aza-pentadecan-14-olide (53)

Reaction of 22 with 3-(6-methoxyquinolin-4-yl)propanal gave 18-dimethylacetal of $\mathbf{5 3}$ in $90 \%$ yield by a similar procedure to 18-dimethylacetal of $\mathbf{5 1}$.

18-Dimethylacetal of 53: $[\alpha]_{\mathrm{D}}^{21}-60^{\circ}\left(c 0.50, \mathrm{CHCl}_{3}\right)$; FAB-MS $m / z 1092(\mathrm{M}+\mathrm{H})^{+}$as $\mathrm{C}_{56} \mathrm{H}_{89} \mathrm{~N}_{3} \mathrm{O}_{18} ;{ }^{1} \mathrm{H}-\mathrm{NMR}$ $\left(300 \mathrm{MHz}, \mathrm{CDCl}_{3}\right) \delta: 0.94(\mathrm{~d}, 18-\mathrm{H}), 1.07$ (d, 6"-H), $1.11(\mathrm{t}$, $\left.3-\mathrm{OCOCH}_{2} \mathrm{CH}_{3}\right), 1.18\left(\mathrm{t}, 4^{\prime \prime}-\mathrm{OCOCH}_{2} \mathrm{CH}_{3}\right), 1.19\left(\mathrm{~d}, 6^{\prime}-\mathrm{H}\right)$, $1.23(\mathrm{~d}, 15-\mathrm{H}), 1.40\left(\mathrm{~s}, 3^{\prime \prime}-\mathrm{CH}_{3}\right), 1.42$ (br dd, 7-H), 1.59 (m, 8-H), 1.68 (dd, 2"-Hax), 1.94 (m, quinoline- $\left.\left(\mathrm{CH}_{2}\right)_{3}\right), 2.00$ (s, 3"-OCOCH $\left.{ }_{3}\right), 2.53\left(\mathrm{~s}, 3^{\prime}-\mathrm{N}\left(\mathrm{CH}_{3}\right)_{2}\right), 2.81(\mathrm{dd}, 2-\mathrm{H}), 3.04$ (m, quinoline- $\left.\left(\mathrm{CH}_{2}\right)_{3}\right), 3.10\left(\mathrm{~s}, 17-\mathrm{OCH}_{3}\right), 3.21\left(\mathrm{~m}, 4^{\prime}-\mathrm{H}\right)$, 3.21 (m, 5'-H), 3.21 (d, 2"-Heq), 3.24 (s, 17- $\left.\mathrm{OCH}_{3}\right), 3.38$ (dd, 2'-H), 3.57 (br d, 4-H), 3.59 (s, 4-- $\mathrm{OCH}_{3}$ ), 3.89 (br d, 5$\mathrm{H}), 3.94$ (s, quinoline-OCH$\left.)_{3}\right), 4.48\left(\mathrm{~d}, 1^{\prime}-\mathrm{H}\right), 4.50\left(\mathrm{dq}, 5^{\prime \prime}-\right.$ H), 4.57 (d, 4"-H), 4.80 (ddq, 14-H), 4.84 (d, 1"-H), 5.19 (br dd, 3-H), 7.18 (d, quinoline), 7.21 (d, quinoline), 7.35 (dd, quinoline), 8.00 (d, quinoline), 8.65 (d, quinoline).

Reaction of 18-dimethylacetal of $\mathbf{5 3}$ with aqueous difluoroacetic acid gave $\mathbf{5 3}$ in $\mathbf{7 6 \%}$ yield by a similar procedure to 11 .

53: $[\alpha]_{\mathrm{D}}^{18}-76^{\circ}\left(c \quad 0.50, \mathrm{CHCl}_{3}\right)$; FAB-MS $m / z 1046$ $(\mathrm{M}+\mathrm{H})^{+}$as $\mathrm{C}_{54} \mathrm{H}_{83} \mathrm{~N}_{3} \mathrm{O}_{17},{ }^{1} \mathrm{H}-\mathrm{NMR}\left(300 \mathrm{MHz}, \mathrm{CDCl}_{3}\right) \delta$ : 0.95 (d, 18-H), 1.08 (d, 6"-H), 1.13 (d, 6'-H), 1.15 (t, 3$\left.\mathrm{OCOCH}_{2} \mathrm{CH}_{3}\right), 1.18$ (t, 4"-OCOCH$\left.{ }_{2} \mathrm{CH}_{3}\right), 1.24$ (d, 15-H), 1.40 (s, 3"- $\left.\mathrm{CH}_{3}\right), 1.44$ (m, 8-H), 1.69 (dd, 2"-Hax), 1.95 (m, quinoline- $\left.\left(\mathrm{CH}_{2}\right)_{3}\right), 1.99\left(\mathrm{~s}, 3 "-\mathrm{OCOCH}_{3}\right), 2.54(\mathrm{~s}, 3$ '$\left.\mathrm{N}\left(\mathrm{CH}_{3}\right)_{2}\right), 2.80(\mathrm{dd}, 2-\mathrm{H}), 2.96(\mathrm{dd}, 16-\mathrm{H}), 3.03(\mathrm{~m}$, quinoline- $\left.\left(\mathrm{CH}_{2}\right)_{3}\right), 3.21\left(\mathrm{~m}, 4^{\prime}-\mathrm{H}\right), 3.21\left(\mathrm{~m}, 5^{\prime}-\mathrm{H}\right), 3.21(\mathrm{~d}$, 2"-Heq), 3.31 (dd, 2'-H), 3.51 (br d, 9-H), 3.58 (s, 4- $\mathrm{OCH}_{3}$ ), 3.63 (dd, 4-H), 3.87 (br d, 5-H), 3.94 (s, quinoline- $\mathrm{OCH}_{3}$ ), 
4.43 (d, 1'-H), 4.48 (dq, 5"'-H), 4.57 (d, 4"'-H), 4.77 (ddq, 14-H), 4.85 (d, 1"-H), 5.31 (br dd, 3-H), 7.18 (d, quinoline), 7.21 (d, quinoline), 7.35 (dd, quinoline), 8.00 (d, quinoline), 8.65 (d, quinoline), 9.63 (s, 17-H).

(-)-(3R,4S,5S,6R,8R,14R)-5-[4-O-(3-O-Acetyl-2,6dideoxy-3-C-methyl-4- $O$-propionyl- $\alpha$-L-ribo-hexopyranosyl)-3,6-dideoxy-3-dimethylamino- $\beta$-D-glucopyranosyloxy]-6-formylmethyl-4-methoxy-8-methyl-11-( $N$-methyl$N$-(3-phenylpropyl)amino)-3-propionyloxy-11-aza-pentadecan-14-olide (54)

Reaction of 12 with 1-methyl-1-(3-phenylpropyl)hydrazine gave 2 -acetate 18 -dimethylacetal of $\mathbf{5 4}$ in $8.6 \%$ yield by a similar procedure to $\mathbf{1 6}$. In the course of this macrocyclization reaction, the acetoxy group at the C-9 position was unexpectedly reduced.

$2^{\prime}$-Acetate 18-dimethylacetal of 54: $[\alpha]_{\mathrm{D}}^{25}-71^{\circ}(c)$ 1.0, $\mathrm{CHCl}_{3}$ ); FAB-MS $m / z 1066(\mathrm{M}+\mathrm{H})^{+}$as $\mathrm{C}_{55} \mathrm{H}_{91} \mathrm{~N}_{3} \mathrm{O}_{17} ;{ }^{1} \mathrm{H}-$ NMR (300 MHz, $\left.\mathrm{CDCl}_{3}\right) \delta$ : 0.90 (d, 18-H), 1.07 (d, 6"-H), $1.14\left(\mathrm{t}, 3-\mathrm{OCOCH}_{2} \mathrm{CH}_{3}\right), 1.19$ (t, 4"-OCOCH $\mathrm{CH}_{3}$ ), 1.20 (d, 6'-H), 1.24 (d, 15-H), $1.42\left(\mathrm{~s}, 3^{\prime \prime}-\mathrm{CH}_{3}\right), 1.46$ (br dd, 16H), 1.67 (dd, 2"-Hax), 1.81 (m, Ph( $\left.\left(\mathrm{CH}_{2}\right)_{3}\right), 2.02$ (s, 3"$\left.\mathrm{OCOCH}_{3}\right), 2.04\left(\mathrm{~s}, 2^{\prime}-\mathrm{OCOCH}_{3}\right), 2.25\left(\mathrm{~s}, 11-\mathrm{NCH}_{3}\right), 2.44$ (s, 3'-N( $\left.\left.\mathrm{CH}_{3}\right)_{2}\right), 3.12\left(\mathrm{~s}, 17-\mathrm{OCH}_{3}\right), 3.19$ (d, 2"-Heq), 3.25 (s, 17- $\mathrm{OCH}_{3}$ ), 3.27 (br d, 4-H), 3.55 (s, 4- $\left.-\mathrm{OCH}_{3}\right), 3.81$ (br s, 5-H), 4.44 (dd, 17-H), 4.49 (dq, 5"-H), 4.57 (d, 4"-H), 4.66 (d, 1'-H), 4.81 (d, 1"-H), 4.88 (m,14-H), 4.96 (dd, 2'-H), 5.36 (br dd, 3-H), $7.19\left(\mathrm{~m}, \mathrm{C}_{6} \mathrm{H}_{5}\right), 7.27\left(\mathrm{~m}, \mathrm{C}_{6} \mathrm{H}_{5}\right)$.

Reaction of $2^{\prime}$-acetate 18-dimethylacetal of $\mathbf{5 4}$ with methanol gave 18-dimethylacetal of $\mathbf{5 4}$ in $69 \%$ yield by a similar procedure to $\mathbf{2 6}$.

18-Dimethylacetal of 54: $[\alpha]_{\mathrm{D}}^{26}-58^{\circ}\left(\mathrm{c} 1.0, \mathrm{CHCl}_{3}\right)$; FAB-MS $m / z 1024(\mathrm{M}+\mathrm{H})^{+}$as $\mathrm{C}_{53} \mathrm{H}_{89} \mathrm{~N}_{3} \mathrm{O}_{16} ;{ }^{1} \mathrm{H}-\mathrm{NMR}$ $\left(300 \mathrm{MHz}, \mathrm{CDCl}_{3}\right) \delta: 0.90(\mathrm{~d}, 18-\mathrm{H}), 1.09\left(\mathrm{~d}, 6^{\prime \prime}-\mathrm{H}\right), 1.15(\mathrm{t}$, $\left.3-\mathrm{OCOCH}_{2} \mathrm{CH}_{3}\right), 1.20$ (t, 4"-OCOCH $\left.\mathrm{CH}_{3}\right), 1.23\left(\mathrm{~d}, 6^{\prime}-\mathrm{H}\right)$, $1.24(\mathrm{~d}, 15-\mathrm{H}), 1.42\left(\mathrm{~s}, 3^{\prime \prime}-\mathrm{CH}_{3}\right), 1.59(\mathrm{~m}, 7-\mathrm{H}), 1.69$ (dd, 2"-Hax), 1.80 (quint, $\left.\mathrm{Ph}\left(\mathrm{CH}_{2}\right)_{3}\right), 2.03\left(\mathrm{~s}, 3^{\prime \prime}-\mathrm{OCOCH}_{3}\right), 2.25$ $\left(\mathrm{s}, 11-\mathrm{NCH}_{3}\right), 2.55\left(\mathrm{~s}, 3^{\prime}-\mathrm{N}\left(\mathrm{CH}_{3}\right)_{2}\right), 3.18\left(\mathrm{~s}, 17-\mathrm{OCH}_{3}\right)$, 3.22 (d, 2"'-Heq), 3.27 (s, 17- $\left.-\mathrm{OCH}_{3}\right), 3.47$ (dd, 2'-H), 3.58 (s, 4- $\left.\mathrm{OCH}_{3}\right), 3.83$ (br d, 5-H), $4.43\left(\mathrm{~d}, 1^{\prime}-\mathrm{H}\right), 4.56$ (m, 5"H), 4.57 (d, 4"-H), 4.85 (d, 1"-H), $4.94(\mathrm{~m}, 14-\mathrm{H}), 5.40$ (m, 3-H), $7.19\left(\mathrm{~m}, \mathrm{C}_{6} \mathrm{H}_{5}\right), 7.27\left(\mathrm{~m}, \mathrm{C}_{6} \mathrm{H}_{5}\right)$.

Reaction of 18 -dimethylacetal of $\mathbf{5 4}$ with aqueous difluoroacetic acid gave $\mathbf{5 4}$ in $76 \%$ yield by a similar procedure to 11.

54: $[\alpha]_{\mathrm{D}}^{25}-70^{\circ}\left(c \quad 0.61, \mathrm{CHCl}_{3}\right)$; FAB-MS $\mathrm{m} / \mathrm{z} 978$ $(\mathrm{M}+\mathrm{H})^{+}{ }^{-}$as $\mathrm{C}_{51} \mathrm{H}_{83} \mathrm{~N}_{3} \mathrm{O}_{15} ;{ }^{1} \mathrm{H}-\mathrm{NMR}\left(300 \mathrm{MHz}, \mathrm{CDCl}_{3}\right) \delta$ : $0.91(\mathrm{~d}, 18-\mathrm{H}), 1.09$ (d, 6"-H), $1.15\left(\mathrm{~d}, 6^{\prime}-\mathrm{H}\right), 1.18$ (t, 3$\left.\mathrm{OCOCH}_{2} \mathrm{CH}_{3}\right), 1.20$ (t, 4"-OCOCH $\left.\mathrm{CH}_{3}\right), 1.25(\mathrm{~d}, 15-\mathrm{H})$, $1.31(\mathrm{~m}, 8-\mathrm{H}), 1.42\left(\mathrm{~s}, 3^{\prime \prime}-\mathrm{CH}_{3}\right), 1.69$ (dd, 2"-Hax), 1.80 (quint, $\left.\mathrm{Ph}\left(\mathrm{CH}_{2}\right)_{3}\right), 2.02\left(\mathrm{~s}, 3^{\prime \prime}-\mathrm{OCOCH}_{3}\right), 2.22$ (brd, 16-H), $2.26\left(\mathrm{~s}, 11-\mathrm{NCH}_{3}\right), 2.55\left(\mathrm{~s}, 3^{\prime}-\mathrm{N}\left(\mathrm{CH}_{3}\right)_{2}\right), 3.02(\mathrm{dd}, 17-\mathrm{H})$, 3.22 (m, 4'-H), 3.22 (d, 2"-Heq), 3.23 (m, 5'-H), 3.39 (br d, 4-H), 3.43 (dd, 2'-H), 3.57 (s, 4-OCH ${ }_{3}$ ), 3.87 (br d, 5-H), 4.46 (d, 1'-H), 4.54 (dq, 5"-H), 4.59 (d, 4"-H), 4.85 (d, 1"$\mathrm{H}), 4.90(\mathrm{~m}, 14-\mathrm{H}), 5.54$ (br dd, 3-H), $7.19\left(\mathrm{~m}, \mathrm{C}_{6} \mathrm{H}_{5}\right), 7.28$ $\left(\mathrm{m}, \mathrm{C}_{6} \mathrm{H}_{5}\right), 9.63(\mathrm{~s}, 17-\mathrm{H})$.

(-)-(3R,4S,5S,6R,8R,9R,14R)-5-[4-O-(3-O-Acetyl-2,6dideoxy-3-C-methyl-4- $O$-propionyl- $\alpha$-L-ribo-hexopyranosyl)-3,6-dideoxy-3-dimethylamino- $\beta$-D-glucopyranosyloxy]-6-formylmethyl-9-hydroxy-4-methoxy-8-methyl-11(trans-3-phenyl-2-propenyl)-3-propionyloxy-11-aza-pentadecan-14-olide (55)

Reaction of 22 with trans-cinnamaldehyde gave 18dimethylacetal of $\mathbf{5 5}$ in $71 \%$ yield by a similar procedure to 18-dimethylacetal of $\mathbf{5 1}$.

18-Dimethylacetal of 55: $[\alpha]_{\mathrm{D}}^{21}-66^{\circ}\left(c 0.50, \mathrm{CHCl}_{3}\right)$; FAB-MS $m / z 1009(\mathrm{M}+\mathrm{H})^{+}$as $\mathrm{C}_{52} \mathrm{H}_{84} \mathrm{~N}_{2} \mathrm{O}_{17} ;{ }^{1} \mathrm{H}-\mathrm{NMR}$ $\left(300 \mathrm{MHz}, \mathrm{CDCl}_{3}\right) \delta: 0.94(\mathrm{~d}, 18-\mathrm{H}), 1.07\left(\mathrm{~d}, 6^{\prime \prime}-\mathrm{H}\right), 1.12(\mathrm{t}$, $3-\mathrm{OCOCH}_{2} \mathrm{CH}_{3}$ ), 1.18 (t, 4"-OCOCH $\left.\mathrm{CH}_{3}\right), 1.19$ (d, 6'-H), $1.25(\mathrm{~d}, 15-\mathrm{H}), 1.40\left(\mathrm{~s}, 3^{\prime \prime}-\mathrm{CH}_{3}\right), 1.44(\mathrm{brdd}, 7-\mathrm{H}), 1.56$ (m, 8-H), 1.68 (dd, 2"-Hax), 2.00 (s, 3"-OCOCH ${ }_{3}$ ), 2.53 (s, 3'$\left.\mathrm{N}\left(\mathrm{CH}_{3}\right)_{2}\right), 2.81$ (dd, 2-H), $3.12\left(\mathrm{~s}, 17-\mathrm{OCH}_{3}\right), 3.20$ (m, 4'$\mathrm{H}), 3.20$ (m, 5'-H), 3.21 (d, 2"-Heq), 3.25 (s, 17- $\left.\mathrm{OCH}_{3}\right)$, 3.40 (dd, 2'-H), 3.59 (s, 4-OCH 3 ), 3.60 (br d, 4-H), 3.88 (br d, 5-H), 4.47 (d, 1'-H), 4.50 (dq, 5"-H), 4.57 (d, 4"-H), 4.82 (ddq, 14-H), 4.84 (d, 1"-H), 5.23 (br dd, 3-H), 6.20 (dt, $\mathrm{PhCH}=\mathrm{CH}), 6.50(\mathrm{~d}, \mathrm{PhCH}=\mathrm{CH}), 7.26\left(\mathrm{~m}, \mathrm{C}_{6} \mathrm{H}_{5}\right)$.

Reaction of 18-dimethylacetal of $\mathbf{5 5}$ with aqueous difluoroacetic acid gave $\mathbf{5 5}$ in $87 \%$ yield by a similar procedure to 11.

55: $[\alpha]_{\mathrm{D}}^{20}-78^{\circ}\left(c \quad 0.40, \mathrm{CHCl}_{3}\right.$ ); FAB-MS $\mathrm{m} / \mathrm{z} 963$ $(\mathrm{M}+\mathrm{H})^{+}$as $\mathrm{C}_{50} \mathrm{H}_{78} \mathrm{~N}_{2} \mathrm{O}_{16} ;{ }^{1} \mathrm{H}-\mathrm{NMR}\left(300 \mathrm{MHz}, \mathrm{CDCl}_{3}\right) \delta$ : 0.96 (d, 18-H), 1.08 (d, 6"-H), 1.13 (d, 6'-H), 1.15 (t, 3$\left.\mathrm{OCOCH}_{2} \mathrm{CH}_{3}\right), 1.18$ (t, 4"-OCOCH $\left.\mathrm{CH}_{3}\right), 1.25$ (d, 15-H), 1.40 (s, 3"--CH $), 1.44$ (m, 8-H), 1.68 (dd, 2"-Hax), 1.99 (s, $\left.3^{\prime \prime}-\mathrm{OCOCH}_{3}\right), 2.54\left(\mathrm{~s}, 3^{\prime}-\mathrm{N}\left(\mathrm{CH}_{3}\right)_{2}\right), 2.80(\mathrm{dd}, 2-\mathrm{H}), 2.95$ (dd, 16-H), $3.21\left(\mathrm{~m}, 4^{\prime}-\mathrm{H}\right), 3.21\left(\mathrm{~m}, 5^{\prime}-\mathrm{H}\right), 3.21\left(\mathrm{~d}, 2^{\prime \prime}-\right.$ Heq), 3.30 (dd, 2'-H), 3.52 (br d, 9-H), 3.58 (s, 4- $\mathrm{OCH}_{3}$ ), 3.66 (dd, 4-H), 3.87 (br d, 5-H), 4.42 (d, 1'-H), 4.49 (dq, 5"H), 4.57 (d, 4"-H), 4.79 (ddq, 14-H), 4.84 (d, 1"-H), 5.36 (br dd, 3-H), $6.19(\mathrm{dt}, \mathrm{PhCH}=\mathrm{CH}), 6.50(\mathrm{~d}, \mathrm{PhCH}=\mathrm{CH})$, $7.26\left(\mathrm{~m}, \mathrm{C}_{6} \mathrm{H}_{5}\right), 9.63(\mathrm{~s}, 17-\mathrm{H})$.

(-)-(3R,4S,5S,6R,8R,9R,14R)-5-[4-O-(3-O-Acetyl-2,6dideoxy-3-C-methyl-4- $O$-propionyl- $\alpha$-L-ribo-hexopyranosyl)-3,6-dideoxy-3-dimethylamino- $\beta$-D-glucopyranosyloxy]-6-formylmethyl-9-hydroxy-4-methoxy-8-methyl-11(3-phenyl-2-propynyl)-3-propionyloxy-11-aza-pentadecan14-olide (56)

Reaction of $\mathbf{2 2}$ with phenylpropargyl aldehyde gave 18- 
dimethylacetal of $\mathbf{5 6}$ in $59 \%$ yield by a similar procedure to 18-dimethylacetal of $\mathbf{5 1}$.

18-Dimethylacetal of 56: $[\alpha]_{\mathrm{D}}^{20}-67^{\circ}\left(c 0.40, \mathrm{CHCl}_{3}\right)$; FAB-MS $m / z 1007(\mathrm{M}+\mathrm{H})^{+}$as $\mathrm{C}_{52} \mathrm{H}_{82} \mathrm{~N}_{2} \mathrm{O}_{17} ;{ }^{1} \mathrm{H}-\mathrm{NMR}$ $\left(300 \mathrm{MHz}, \mathrm{CDCl}_{3}\right) \delta: 0.95(\mathrm{~d}, 18-\mathrm{H}), 1.07\left(\mathrm{~d}, 6^{\prime \prime}-\mathrm{H}\right), 1.13(\mathrm{t}$, $\left.3-\mathrm{OCOCH}_{2} \mathrm{CH}_{3}\right), 1.18$ (t, 4"-OCOCH$\left.{ }_{2} \mathrm{CH}_{3}\right), 1.19\left(\mathrm{~d}, 6^{\prime}-\mathrm{H}\right)$, 1.27 (d, 15-H), 1.40 (s, 3"--CH $), 1.47$ (br dd, 7-H), 1.57 (m, 8-H), 1.57 (m, 16-H), 1.68 (dd, 2"-Hax), 1.88 (m, 16-H), $2.00\left(\mathrm{~s}, 3^{\prime \prime}-\mathrm{OCOCH}_{3}\right), 2.53\left(\mathrm{~s}, 3^{\prime}-\mathrm{N}\left(\mathrm{CH}_{3}\right)_{2}\right), 2.79$ (dd, 2-H), 3.16 (s, 17- $\left.-\mathrm{OCH}_{3}\right), 3.20$ (d, 2"-Heq), 3.22 (m, 4'-H), 3.23 (m, 5'-H), $3.25\left(\mathrm{~s}, 17-\mathrm{OCH}_{3}\right), 3.42\left(\mathrm{dd}, 2^{\prime}-\mathrm{H}\right), 3.59$ (s, 4$\mathrm{OCH}_{3}$ ), 3.62 (br d, 4-H), 3.86 (br d, 5-H), 4.44 (d, 1'-H), 4.50 (dq, 5"-H), 4.57 (d, 4"-H), 4.83 (d, 1"-H), 4.97 (ddq, 14-H), 5.27 (br dd, 3-H), 7.29 (m, $\left.\mathrm{C}_{6} \mathrm{H}_{5}\right), 7.42\left(\mathrm{~m}, \mathrm{C}_{6} \mathrm{H}_{5}\right)$.

Reaction of 18-dimethylacetal of $\mathbf{5 6}$ with aqueous difluoroacetic acid gave $\mathbf{5 6}$ in $73 \%$ yield by a similar procedure to 11 .

56: $[\alpha]_{\mathrm{D}}^{23}-71^{\circ}\left(c \quad 0.25, \mathrm{CHCl}_{3}\right)$; FAB-MS $m / z 961$ $(\mathrm{M}+\mathrm{H})^{+}$as $\mathrm{C}_{50} \mathrm{H}_{76} \mathrm{~N}_{2} \mathrm{O}_{16} ;{ }^{1} \mathrm{H}-\mathrm{NMR}\left(300 \mathrm{MHz}, \mathrm{CDCl}_{3}\right) \delta$ : 0.98 (d, 18-H), 1.08 (d, 6"-H), 1.13 (d, 6'-H), 1.15 (t, 3$\mathrm{OCOCH}_{2} \mathrm{CH}_{3}$ ), 1.18 (t, 4"-OCOCH$\left.{ }_{2} \mathrm{CH}_{3}\right), 1.27(\mathrm{~d}, 15-\mathrm{H})$, $1.40\left(\mathrm{~s}, 3^{\prime \prime}-\mathrm{CH}_{3}\right), 1.51(\mathrm{~m}, 8-\mathrm{H}), 1.68$ (dd, 2"-Hax), 1.99 (s, $\left.3^{\prime \prime}-\mathrm{OCOCH}_{3}\right), 2.54\left(\mathrm{~s}, 3^{\prime}-\mathrm{N}\left(\mathrm{CH}_{3}\right)_{2}\right), 2.79$ (dd, 2-H), 2.94 (dd, 16-H), 3.21 (d, 2"-Heq), 3.21 (m, 4'-H), 3.21 (m, 5' H), 3.35 (dd, 2'-H), 3.58 (s, 4-OCH ${ }_{3}$ ), 3.61 (br d, 4-H), 3.87 (br d, 5-H), 4.41 (d, 1'-H), 4.49 (dq, 5"-H), 4.57 (d, 4"-H), 4.84 (d, 1"-H), 4.90 (ddq, 14-H), 5.41 (br dd, 3-H), 7.29 (m, $\left.\mathrm{C}_{6} \mathrm{H}_{5}\right), 7.42\left(\mathrm{~m}, \mathrm{C}_{6} \mathrm{H}_{5}\right), 9.64(\mathrm{~s}, 17-\mathrm{H})$.

(-)-(3R,4S,5S,6R,8R,9R,14R)-5-[4-O-(3-O-Acetyl-2,6dideoxy-3-C-methyl-4- $O$-propionyl- $\alpha$-L-ribo-hexopyranosyl)-3,6-dideoxy-3-dimethylamino- $\beta$-D-glucopyranosyloxy]-6-formylmethyl-9-hydroxy-4-methoxy-8-methyl-11(3-phenylpropionyl)-3-propionyloxy-11-aza-pentadecan14-olide (57)

To a solution of $22(30.0 \mathrm{mg})$ in dichloromethane $(0.60 \mathrm{ml})$, triethylamine $(17 \mu \mathrm{l})$ and 3-phenylpropionyl chloride $(6 \mu \mathrm{l})$ were added under ice cooling, and the mixture was stirred for 1 hour. Chloroform $(5.0 \mathrm{ml})$ and water $(5.0 \mathrm{ml})$ were added to the reaction mixture, and the organic layer was separated. Then the aqueous layer was extracted twice with chloroform $(10 \mathrm{ml})$. The organic layers were combined, washed successively with saturated aqueous sodium hydrogencarbonate solution $(10 \mathrm{ml})$ and saturated brine $(10 \mathrm{ml})$, dried over anhydrous sodium sulfate, and then filtered. The filtrate was concentrated under reduced pressure, and the resulting residue was purified by preparative TLC (ethyl acetate/methanol/aqueous ammonia $(30: 1: 0.1)$ and chloroform/methanol/aqueous ammonia $(20: 1: 0.1))$ to obtain 18 -dimethylacetal of $57(20.0 \mathrm{mg}$, $58 \%)$.
18-Dimethylacetal of 57: $[\alpha]_{\mathrm{D}}^{21}-26^{\circ}\left(c 0.40, \mathrm{CHCl}_{3}\right)$; FAB-MS $m / z 1025(\mathrm{M}+\mathrm{H})^{+}$as $\mathrm{C}_{52} \mathrm{H}_{84} \mathrm{~N}_{2} \mathrm{O}_{18} ;{ }^{1} \mathrm{H}-\mathrm{NMR}$ $\left(300 \mathrm{MHz}, \mathrm{CDCl}_{3}\right) \delta: 0.99(\mathrm{~d}, 18-\mathrm{H}), 1.08$ (d, 6"-H), 1.09 (t, $3-\mathrm{OCOCH}_{2} \mathrm{CH}_{3}$ ), 1.18 (t, 4"-OCOCH${ }_{2} \mathrm{CH}_{3}$ ), 1.19 (d, 6'-H), 1.21 (d, 15-H), 1.41 (s, 3"- $\left.\mathrm{CH}_{3}\right), 1.50$ (br dd, 7-H), 1.69 (dd, 2"-Hax), 1.74 (m, 8-H), 1.99 (s, 3"-OCOCH ${ }_{3}$ ), 2.54 (s, 3'$\left.\mathrm{N}\left(\mathrm{CH}_{3}\right)_{2}\right), 2.78$ (dd, 2-H), 3.07 (s, 17- $\left.\mathrm{OCH}_{3}\right), 3.21$ (s, 17$\mathrm{OCH}_{3}$ ), 3.22 (d, 2"-Heq), 3.23 (m, 4'-H), 3.24 (m, 5'-H), 3.34 (dd, 2'-H), 3.50 (br d, 4-H), 3.61 (s, 4- $\left.-\mathrm{OCH}_{3}\right), 3.78$ (br d, 9-H), 3.89 (br d, 5-H), 4.51 (dq, 5"-H), 4.54 (ddq, 14H), 4.55 (d, 1'-H), 4.58 (d, 4"-H), 4.85 (d, 1"-H), 5.14 (br dd, 3-H), $7.20\left(\mathrm{~m}, \mathrm{C}_{6} \mathrm{H}_{5}\right), 7.28\left(\mathrm{~m}, \mathrm{C}_{6} \mathrm{H}_{5}\right)$.

Reaction of 18-dimethylacetal of 57 with aqueous difluoroacetic acid gave $\mathbf{5 7}$ in $\mathbf{7 9 \%}$ yield by a similar procedure to $\mathbf{1 1}$.

57: $[\alpha]_{\mathrm{D}}^{20}-37^{\circ}\left(c 0.40, \mathrm{CHCl}_{3}\right)$; FAB-MS $m / z 979$ $(\mathrm{M}+\mathrm{H})^{+}$as $\mathrm{C}_{50} \mathrm{H}_{78} \mathrm{~N}_{2} \mathrm{O}_{17}{ }^{1} \mathrm{H}-\mathrm{NMR}\left(300 \mathrm{MHz}, \mathrm{CDCl}_{3}\right) \delta$ : 0.99 (d, 18-H), 1.08 (d, 6"-H), 1.11 (d, 6'-H), 1.14 (t, 3$\mathrm{OCOCH}_{2} \mathrm{CH}_{3}$ ), 1.18 (t, 4"-OCOCH $\left.\mathrm{CH}_{3}\right), 1.22$ (d, 15-H), 1.40 (s, 3"- $\left.\mathrm{CH}_{3}\right), 1.53$ (m, 8-H), 1.69 (dd, 2"-Hax), 1.98 (s, 3"-OCOCH$\left.{ }_{3}\right), 2.55\left(\mathrm{~s}, 3^{\prime}-\mathrm{N}\left(\mathrm{CH}_{3}\right)_{2}\right), 2.76(\mathrm{dd}, 2-\mathrm{H}), 2.89$ (dd, 16-H), 3.21 (d, 2"-Heq), 3.22 (m, 4'-H), 3.22 (m, 5'$\mathrm{H}), 3.28\left(\mathrm{dd}, 2^{\prime}-\mathrm{H}\right), 3.46(\mathrm{dd}, 4-\mathrm{H}), 3.61\left(\mathrm{~s}, 4-\mathrm{OCH}_{3}\right), 3.73$ (br d, 9-H), 3.89 (br d, 5-H), 4.44 (d, 1'-H), 4.47 (dq, 5"-H), 4.50 (ddq, 14-H), 4.58 (d, 4"-H), 4.85 (d, 1"-H), 5.22 (br dd, 3-H), $7.20\left(\mathrm{~m}, \mathrm{C}_{6} \mathrm{H}_{5}\right), 7.28\left(\mathrm{~m}, \mathrm{C}_{6} \mathrm{H}_{5}\right), 9.59(\mathrm{~s}, 17-\mathrm{H})$.

(-)-(3R,4S,5S,6R,8R,9R,14R)-5-[4-O-(3-O-Acetyl-2,6dideoxy-3-C-methyl-4- $O$-propionyl- $\alpha$-L-ribo-hexopyranosyl)-3,6-dideoxy-3-dimethylamino- $\beta$-D-glucopyranosyloxy]-11-(3-(2-benzyloxyphenyl)propyl)-6-formylmethyl9-hydroxy-4-methoxy-8-methyl-3-propionyloxy-11-azapentadecan-14-olide (58)

Reaction of 22 with 3-(2-benzyloxyphenyl)propanal gave 18-dimethylacetal of $\mathbf{5 8}$ in $80 \%$ yield by a similar procedure to 18-dimethylacetal of $\mathbf{5 1}$.

18-Dimethylacetal of 58: $[\alpha]_{\mathrm{D}}^{22}-57^{\circ}\left(c 0.50, \mathrm{CHCl}_{3}\right)$; FAB-MS $m / z 1117(\mathrm{M}+\mathrm{H})^{+}$as $\mathrm{C}_{59} \mathrm{H}_{92} \mathrm{~N}_{2} \mathrm{O}_{18} ;{ }^{1} \mathrm{H}-\mathrm{NMR}$ $\left(300 \mathrm{MHz}, \mathrm{CDCl}_{3}\right) \delta: 0.93(\mathrm{~d}, 18-\mathrm{H}), 1.08\left(\mathrm{~d}, 6^{\prime \prime}-\mathrm{H}\right), 1.11(\mathrm{t}$, $\left.3-\mathrm{OCOCH}_{2} \mathrm{CH}_{3}\right), 1.18\left(\mathrm{t}, 4^{\prime \prime}-\mathrm{OCOCH} \mathrm{CH}_{3}\right), 1.20\left(\mathrm{~d}, 6^{\prime}-\mathrm{H}\right)$, $1.20(\mathrm{~d}, 15-\mathrm{H}), 1.41\left(\mathrm{~s}, 3^{\prime \prime}-\mathrm{CH}_{3}\right), 1.64(\mathrm{~m}, 16-\mathrm{H}), 1.68(\mathrm{dd}$, 2"-Hax), 1.86 ( $\left.\mathrm{m}^{\mathrm{C}} \mathrm{C}_{6} \mathrm{H}_{4}\left(\mathrm{CH}_{2}\right)_{3}\right), 1.90$ (m, 16-H), 2.00 (s, 3"$\left.\mathrm{OCOCH}_{3}\right), 2.53\left(\mathrm{~s}, 3^{\prime}-\mathrm{N}\left(\mathrm{CH}_{3}\right)_{2}\right), 2.81(\mathrm{dd}, 2-\mathrm{H}), 3.11$ (s, $17-\mathrm{OCH}_{3}$ ), 3.20 (m, 4'-H), 3.20 (m, 5'-H), 3.21 (d, 2"-Heq), 3.25 (s, 17- $\left.-\mathrm{OCH}_{3}\right), 3.40$ (dd, 2'-H), 3.56 (br d, 4-H), 3.58 (s, 4- $\left.\mathrm{OCH}_{3}\right), 3.88$ (br d, 5-H), 4.47 (d, 1'-H), 4.51 (dq, 5"-H), 4.58 (d, 4"-H), 4.75 (ddq, 14-H), 4.84 (d, 1"-H), 5.07 (s, $\mathrm{C}_{6} \mathrm{H}_{5} \mathrm{CH}_{2}$ ), 5.19 (br dd, 3-H), $6.90\left(\mathrm{~m}, \mathrm{C}_{6} \mathrm{H}_{4}\right), 7.15$ (m, $\left.\mathrm{C}_{6} \mathrm{H}_{4}\right), 7.36\left(\mathrm{~m}, \mathrm{C}_{6} \mathrm{H}_{5}\right)$.

Reaction of 18-dimethylacetal of $\mathbf{5 8}$ with aqueous difluoroacetic acid gave $\mathbf{5 8}$ in $76 \%$ yield by a similar 
procedure to 11.

58: $[\alpha]_{\mathrm{D}}^{19}-73^{\circ}\left(c 0.50, \mathrm{CHCl}_{3}\right)$; FAB-MS $m / z 1071$ $(\mathrm{M}+\mathrm{H})^{+}$as $\mathrm{C}_{57} \mathrm{H}_{86} \mathrm{~N}_{2} \mathrm{O}_{17} ;{ }^{1} \mathrm{H}-\mathrm{NMR}\left(300 \mathrm{MHz}, \mathrm{CDCl}_{3}\right) \delta$ : 0.95 (d, 18-H), 1.08 (d, 6"-H), 1.13 (d, 6'-H), 1.15 (t, 3$\left.\mathrm{OCOCH}_{2} \mathrm{CH}_{3}\right), 1.18$ (t, 4"-OCOCH $\left.\mathrm{CH}_{3}\right), 1.20(\mathrm{~d}, 15-\mathrm{H})$, 1.41 (s, 3"- $\left.\mathrm{CH}_{3}\right), 1.46$ (m, 8-H), 1.69 (dd, 2"-Hax), 1.77 (m, $\left.\mathrm{C}_{6} \mathrm{H}_{4}\left(\mathrm{CH}_{2}\right)_{3}\right), 1.99$ (s, 3"'-OCOCH $\left.{ }_{3}\right), 2.54$ (s, 3'- $\left.\left(\mathrm{CH}_{3}\right)_{2}\right)$, $2.66\left(\mathrm{~m}, \mathrm{C}_{6} \mathrm{H}_{4}\left(\mathrm{CH}_{2}\right)_{3}\right), 2.79$ (dd, 2-H), 2.95 (dd, 16-H), 3.21 (m, 4'-H), 3.21 (m, 5'-H), 3.21 (d, 2"-Heq), 3.33 (dd, 2'-H), 3.57 (s, 4- $\left.-\mathrm{OCH}_{3}\right), 3.63$ (dd, 4-H), 3.87 (br d, 5-H), 4.42 (d, 1'-H), 4.48 (dq, 5"-H), 4.58 (d, 4"-H), 4.71 (ddq, 14-H), 4.84 (d, 1"-H), 5.07 (s, $\mathrm{C}_{6} \mathrm{H}_{5} \mathrm{CH}_{2}$ ), 5.31 (br dd, 3-H), 6.88 (m, $\left.\mathrm{C}_{6} \mathrm{H}_{4}\right), 7.15\left(\mathrm{~m}, \mathrm{C}_{6} \mathrm{H}_{4}\right), 7.36\left(\mathrm{~m}, \mathrm{C}_{6} \mathrm{H}_{5}\right), 9.63(\mathrm{~s}, 17-\mathrm{H})$.

(-)-(3R,4S,5S,6R,8R,9R,14R)-5-[4-O-(3-O-Acetyl-2,6dideoxy-3-C-methyl-4- $O$-propionyl- $\alpha$-L-ribo-hexopyranosyl)-3,6-dideoxy-3-dimethylamino- $\beta$-D-glucopyranosyloxy]-6-(2,2-dimethoxyethyl)-9-hydroxy-4-methoxy-8methyl-11-(4-phenylbutyl)-3-propionyloxy-11-aza-pentadecan-14-olide (30c)

$14(150 \mathrm{mg})$ was dissolved in anhydrous methanol $(5.0 \mathrm{ml})$, and ozone was introduced into this solution at $-78^{\circ} \mathrm{C}$ for 15 minutes until getting blue color solution of reaction mixture. Then, oxygen was bubbled for 5 minutes in order to remove excess ozone at the same temperature. Dimethylsulfide $(1.0 \mathrm{ml})$ was added at $-78^{\circ} \mathrm{C}$ and the reaction mixture was kept at the same temperature for 30 minutes. Next, 4-phenylbutyl amine $(28 \mu \mathrm{l})$ and sodium triacetoxyborohydride $(100 \mathrm{mg})$ were added, and the mixture was gradually warmed up to room temperature. About half volume of methanol was evaporated under the reduced pressure, and the resulting solution was neutralized with saturated aqueous sodium bicarbonate solution. Then, the aqueous layer was extracted with ethyl acetate. The organic layer was dried over anhydrous sodium sulfate, and then filtered. The filtrate was concentrated under reduced pressure, and the resulting residue was purified by silica gel column chromatography (hexane/ethyl acetate $(5: 1$ to $1: 1))$ to obtain 30c $(13.0 \mathrm{mg}, 8.0 \%)$.

Acknowledgment The authors wish to thank Drs. Y. Otsuka, T. Okonogi, S. Hoshiko, for encouragement and valuable discussion. We are grateful to Drs. S. Inouye, S. Shibahara and S. Gomi for supervision through our in-house drug discovery program in macrolide field. We also thank Mrs. K. Kobayashi for direction in intellectual properties, Drs. M. Oyama, Y. Takeuchi, K. Kurihara, T. Furuuchi, Mr. N. Okura, Mr. T. Watanabe, Mrs. T. Miyara, and Miss S. Miki for contribution toward analytical and synthetic chemistry, Mr. Y. Takayama for in vitro evaluation, Mrs. Y. Saito for manuscript, and Mrs. M. Takagi for English.
References

1. (a) Ōmura S. (Ed): Macrolide Antibiotics. Chemistry, Biology, and Practice. Academic Press Inc., (1984)

(b) Ōmura S. (Ed): Macrolide Antibiotics. Chemistry, Biology, and Practice. Second Edition, Academic Press Inc., (2002)

2. Morimoto S, Takahashi Y, Watanabe Y, Omura S. Chemical modification of erythromycins. I. Synthesis and antibacterial activity of 6-O-methylerythromycins A. J Antibiot 37: 187-189 (1984)

3. Slobodan D, Gabrijela K, Nevenka L, Boris K, Ante N, Draginja M. Erythromycin series. Part 13. Synthesis and structure elucidation of 10-dihydro-10-deoxo-11-methyl-11azaerythromycin A. J Chem Res Synop 152-153 (1988)

4. Denis A, Agouridas C, Auger JM, Benedetti Y, Bonnefoy A, Bretin F, Chantot JF, Dussarat A, Fromentin C, D'Ambrieres SG, Lachaud S, Laurin P, Martret OL, Loyau V, Tessot N, Pejac JM, Perron S. Synthesis and antibacterial activity of HMR 3647. A new ketolide highly potent against erythromycin-resistant and susceptible pathogens. Bioorg Med Chem Lett 9: 3075-3080 (1999)

5. Or YS, Wang G, Phan LT, Niu D, Vo NH, Qiu YL, Wang Y, Busuyek M, Hou Y, Peng Y, Kim H, Liu T, Farmer JJ, Xu G. (Enanta Pharmaceuticals, Inc.). 6-11 Bicyclic ketolide derivatives. WO 03/097659 A1, Nov. 27 (2003)

6. Mathews AW. Fraud, errors taint key study of Sanofi drug (from The Wall Street Journal). Dow Jones Newswires, May 1, 2006, (C) 2006 Dow Jones \& Company, Inc.

7. (a) Ajito K, Kurihara K, Shibahara S, Hara O, Shimizu A, Araake M, Omoto S. Cladinose analogues of sixteenmembered macrolide antibiotics. II. Preparation of pharmacokinetically improved analogues via biotransformations. J Antibiot 50: 92-95 (1997)

(b) Kurihara K, Kikuchi N, Ajito K. Cladinose analogues of sixteen-membered macrolide antibiotics. III. Efficient synthesis of 4-O-alkyl-L-cladinose analogues: Improved antibacterial activities comparable with pharmacokinetics. J Antibiot 50: 32-44 (1997)

8. (a) Furuuchi T, Kurihara K, Ajito K, Yoshida T, Fushimi H. (Meiji Seika Kaisha, Ltd.). 3-Substituted leucomycins and pharmaceutical compositions containing them. JP 2004217562, Aug. 5 (2004)

(b) Kurihara K, Miura T, Okura N, Yoshida T, Furuuchi T, Ajito K. (Meiji Seika Kaisha, Ltd.). 12- and 13-modified novel 16-membered macrolide derivatives. WO 02/064607 A1, Aug. 22 (2002)

(c) Kurihara K, Miura T, Okura N, Furuuchi T, Fujihira Y, Yoshida T, Fushimi H, Ajito K. (Meiji Seika Kaisha, Ltd.). 12-Oxy-13-amino-cintaining 16-membered cyclic macrolide derivative and process for producing the same. WO 2006/073172 A1, Jul. 13 (2006)

9. Kobrehel G, Djokic S. (Pliva). 11-Methyl-11-aza-4-O cladinosyl-6-O-desosaminyl-15-ethyl-7,13,14-trihydroxy- 
3,5,7,9,12,14-hexamethyl-oxacyclopentadecane-2-one and derivatives thereof. US Patent 4,517,359, May 14 (1985)

10. Waddell ST, Blizzard TA. (Merck \& Co. Inc.). 8a-Aza and 9a-aza macrolide antibiotics, and a process for producing same and methods of use. WO 94/15617, Jul. 21 (1994)

11. Jones AB. New macrolide antibiotics: Synthesis of a 14membered azalide. J Org Chem 57: 4361-4367 (1992)

12. Shankaran K, Wilkening RR. (Merck \& Co. Inc.). Methods of making 4" derivatives of 9-deoxo-8a-aza-8a-alkyl-8ahomoerythromycin A. EP 0549040 A1, Jun. 30 (1993)

13. Lazarevski G, Kobrehel G, Kelneric Z. (Pliva). 15Membered lactams ketolides with antibacterial activity. WO 99/51616, Oct. 14 (1994)

14. Waddell ST, Blizzard TA. Chimeric azalides with simplified western portions. Tetrahedron Lett 34: 5385-5388 (1993)

15. Lopotar N, Djokic S. (Pliva). Tylosin derivatives. EP 0410 433 B1, Feb. 28 (1996)

16. Mutak S. Azalides from azithromycin to new azalide derivatives. J Antibiot 60: 85-122 (2007)

17. Pavlovic D. Synthesis and antibacterial activity of novel 8aaza-8a-homoerythromycin A ketolides: Consequences of structural modification at the C-6 position. J Org Chem in press

18. Or YS, Qiu Y, Wang G, Niu D, Phan LT. (Enanta Pharmaceuticals, Inc.). New bicyclic 9a-azalide derivatives useful for the treatment of bacterial and protozoal infection. US2006069048 A1, Mar. 30 (2006)

19. Berdik A, Kobrehel G, Lazarevski G, Mutak S. (Pliva d.d.). The present invention related to the new 3-decladinosyl derivatives of 9-deoxo-9a-aza-9a-homoerythromycin A 9a,11-cyclic carbamates. WO 2004/029067 A1, Apr. 8 (2004)

20. Asaka T, Manaka A, Tanikawa T, Sugimoto T, Shimazaki Y, Sato M. 11a-Azalide compounds and process for producing the same. WO 03/014136 A1, Feb. 20 (2003)

21. Ōmura S, Miyano K, Matsubara H, Nakagawa A. Novel dimeric derivatives of leucomycins and tylosin, sixteenmembered macrolides. J Med Chem 25: 271-275 (1982)

22. (a) Freiberg LA, Egan RS, Washburn WH. The synthesis of 9-epi-leucomycin $\mathrm{A}_{3}$. The revised configurational assignment of C-9 in natural leucomycin $\mathrm{A}_{3}$. J Org Chem 39: 2474-2475 (1974)

(b) Furuuchi T, Kurihara K, Yoshida T, Ajito K. Synthesis and biological evaluation of novel leucomycin analogues modified at the C-3 position. I. Epimerization and methylation of the 3-hydroxyl group. J Antibiot 56: 399-414 (2003)

23. Omoto S, Iwamatsu K, Inouye S, Niida T. Modifications of a macrolide antibiotic midecamycin (SF-837). I. Synthesis and structure of 9,3"-diacetylmidecamycin. J Antibiot 29: 536-548 (1976)

24. Tsuruoka T, Shomura T. Ezaki N, Watanabe H, Akita E, Inouye S, Niida T. Studies on antibiotic SF-837, a new antibiotic. I. The producing microorganism and isolation and characterization of the antibiotic. J Antibiot 24: 452-459 (1971)

25. Miura T, Kurihara K, Yoshida T, Ajito K. (Meiji Seika Kaisha, Ltd.). Novel 15-membered cyclic azalide, novel 16membered cyclic diazalide derivative, and process for producing these. US 2005/0209446 A1, Sep. 22 (2005)

26. Sakakibara H, Okekawa O, Fujiwara T, Otani M, Omura S. Acyl derivatives of 16-membered macrolides. I. Synthesis and biological properties of 3 "-O-propionylleucomycin $\mathrm{A}_{5}$ (TMS-19-Q). J Antibiot 34: 1001-1010 (1981)

27. Omoto S, Inouye S, Niida T. (Meiji Seika Kaisha, Ltd.). A one step process for the production of a mono-ester of macrolide antibiotics. Japan Kokai 13380 (1973), Feb. 20 (1973)

28. Sano H, Sunazuka T, Tanaka H, Yamashita K, Okachi R, Ōmura S. Chemical modification of spiramycins. III. Synthesis and antibacterial activities of 4"-sulfonates and 4"alkylethers of spiramycin I. J Antibiot 37: 750-759 (1984)

29. Kurihara K, Ajito K, Shibahara S, Hara O, Araake M, Omoto S, Inouye S. Cladinose analogues of sixteenmembered macrolide antibiotics. VI. Synthesis of metabolically programmed, highly potent analogues of sixteen-membered macrolide antibiotics. J Antibiot 51: 771-785 (1998)

30. Sasai H, Arai T, Miura T, Atsumi K, Ajito K. (Meiji Seika Kaisha, Ltd.). Novel method for producing dialdehyde and related compounds. WO 2005/007666 A1, Jan. 27 (2005)

31. Kuo D, Leresche JE, Proplesch R, Roduit JP, Bessard Y, Armbruster E. (Lonza). Process for preparing 1-(6methylpyridin-3-yl)-2-[4-(methylsulfonyl)phenyl]ethanone. US 2005/0159458 A1, Jul. 21 (2005)

32. Kurihara K, Furuuchi T, Yoshida T, Miura T, Ajito K. (Meiji Seika Kaisha, Ltd.). 3-Modified leucomycin derivatives. WO 00/73317 A1, Dec. 7 (2000) 